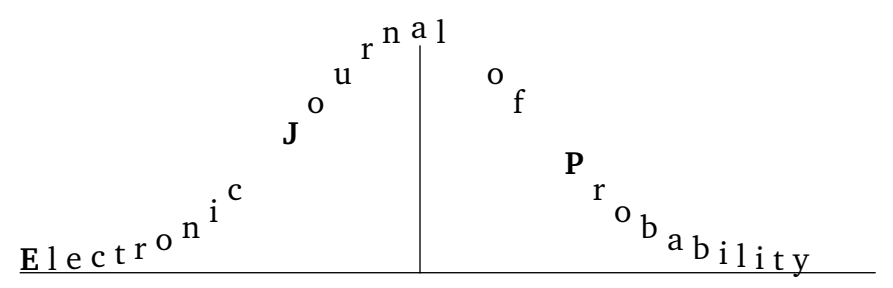

Vol. 15 (2010), Paper no. 18, pages 526-603.

Journal URL

http://www.math.washington.edu/ ejpecp/

\title{
Universality of sine-kernel for Wigner matrices with a small Gaussian perturbation
}

\author{
László Erdős*i José A. Ramírez; Benjamin Schlein ${ }^{\S}$ and Horng-Tzer Yau ${ }^{\top} \|$
}

\begin{abstract}
We consider $N \times N$ Hermitian random matrices with independent identically distributed entries (Wigner matrices). We assume that the distribution of the entries have a Gaussian component with variance $N^{-3 / 4+\beta}$ for some positive $\beta>0$. We prove that the local eigenvalue statistics follows the universal Dyson sine kernel.
\end{abstract}

Key words: Wigner random matrix, Dyson sine kernel.

AMS 2000 Subject Classification: Primary 15A52, 82B44.

Submitted to EJP on June 19, 2009, final version accepted April 2, 2010.

\footnotetext{
*Partially supported by SFB-TR 12 Grant of the German Research Council

'Institute of Mathematics, University of Munich, Theresienstr. 39, D-80333 Munich, Germany, lerdos@math.lmu.de

Department of Mathematics, Universidad de Costa Rica, San Jose 2060, Costa Rica, alexander.ramirezgonzalez@ucr.ac.cr

${ }^{\S}$ Department of Pure Mathematics and Mathematical Statistics, University of Cambridge, Wilberforce Rd, Cambridge CB3 0WB, UK, b.schlein@dpmms.cam.ac.uk

'Department of Mathematics, Harvard University, Cambridge MA 02138, USA, htyau@math.harvard.edu

"Partially supported by NSF grants DMS-0602038, 0757425, 0804279
} 


\section{Introduction}

Certain spectral statistics of broad classes of $N \times N$ random matrix ensembles are believed to follow a universal behavior in the limit $N \rightarrow \infty$. Wigner has observed [30] that the density of eigenvalues of large symmetric or hermitian matrices $H$ with independent entries (up to the symmetry requirement) converges, as $N \rightarrow \infty$, to a universal density, the Wigner semicircle law. Dyson has observed that the local correlation statistics of neighboring eigenvalues inside the bulk of the spectrum follows another universal pattern, the Dyson sine-kernel in the $N \rightarrow \infty$ limit [10]. Moreover, any $k$-point correlation function can be obtained as a determinant of the two point correlation functions. The precise form of the universal two point function in the bulk seems to depend only on the symmetry class of the matrix ensemble (a different universal behavior emerges near the spectral edge [28]).

Dyson has proved this fact for the Gaussian Unitary Ensemble (GUE), where the matrix elements are independent, identically distributed complex Gaussian random variables (subject to the hermitian constraint). A characteristic feature of GUE is that the distribution is invariant under unitary conjugation, $H \rightarrow U^{*} H U$ for any unitary matrix $U$. Dyson found an explicit formula for the joint density function of the $N$ eigenvalues. The formula contains a characteristic Vandermonde determinant and therefore it coincides with the Gibbs measure of a particle system interacting via a logarithmic potential analogously to the two dimensional Coulomb gas. Dyson also observed that the computation of two point function can be reduced to asymptotics of Hermite polynomials.

His approach has later been substantially generalized to include a large class of random matrix ensembles, but always with unitary (orthogonal, symplectic, etc.) invariance. For example, a general class of invariant ensembles can be given by the measure $Z^{-1} \exp (-\operatorname{Tr} V(H)) \mathrm{d} H$ on the space of hermitian matrices, where $\mathrm{d} H$ stands for the Lebesgue measure for all independent matrix entries, $Z$ is the normalization and $V$ is a real function with certain smoothness and growth properties. For example, the GUE ensemble corresponds to $V(x)=x^{2}$.

The joint density function is explicit in all these cases and the evaluation of the two point function can be reduced to certain asymptotic properties of orthogonal polynomials with respect to the weight function $\exp (-V(x))$ on the real line. The sine kernel can thus be proved for a wide range of potentials $V$. Since the references in this direction are enormous, we can only refer the reader to the book by Deift [9] for the Riemann-Hilbert approach, the paper by Levin and Lubinsky [23] and references therein for approaches based on classical analysis of orthogonal polynomials, or the paper by Pastur and Shcherbina [26] for a probabilistic/statistical physics approach. The book by Anderson et al [1] or the book by Metha [25] also contain extensive lists of literatures.

Since the computation of the explicit formula of the joint density relies on the unitary invariance, there have been very little progress in understanding non-unitary invariant ensembles. The most prominent example is the Wigner ensemble or Wigner matrices, i.e., hermitian random matrices with i.i.d. entries. Wigner matrices are not unitarily invariant unless the single entry distribution is Gaussian, i.e. for the GUE case. The disparity between our understanding of the Wigner ensembles and the unitary invariant ensembles is startling. Up until the very recent work of [14], there was no proof that the density follows the semicircle law in small spectral windows unless the number of eigenvalues in the window is at least $\sqrt{N}$. This is entirely due to a serious lack of analytic tools for studying eigenvalues once the mapping between eigenvalues and Coulomb gas ceases to apply. At present, there are only two rigorous approaches to eigenvalue distributions: the moment method and Green function method. The moment method is restricted to studying the spectrum near the edges [28]; the precision of the Green function method seems to be still very far from getting 
information on level spacing [6].

Beyond the unitary ensembles, Johansson [21] proved the sine-kernel for a broader category of ensembles, i.e., for matrices of the form $H+s V$ where $H$ is a Wigner matrix, $V$ is an independent GUE matrix and $s$ is a positive constant of order one. (Strictly speaking, in the original work [21], the range of the parameter $s$ depends on the energy $E$. This restriction was later removed by Ben Arous and Péché [3], who also extended this approach to Wishart ensembles). Alternatively formulated, if the matrix elements are normalized to have variance one, then the distribution of the matrix elements of the ensemble $H+s V$ is given by $v * \mathscr{G}_{s}$, where $v$ is the distribution of the Wigner matrix elements and $\mathscr{G}_{s}$ is the centered Gaussian law with variance $s^{2}$. Johasson's work is based on the analysis of the explicit formula for the joint eigenvalue distribution of the matrix $H+s V$ (see also [7]).

Dyson has introduced a dynamical version of generating random matrices. He considered a matrixvalued process $H+s V$ where $V$ is a matrix-valued Brownian motion. The distribution of the eigenvalues then evolves according to a process called Dyson's Brownian motions. For the convenience of analysis, we replace the Brownian motions by an Ornstein-Uhlenbeck process so that the distribution of GUE is the invariant measure of this modified process, which we still call Dyson's Brownian motion. Dyson's Brownian motion thus can be viewed as a reversible interacting particle system with a long range (logarithmic) interaction. This process is well adapted for studying the evolution of the empirical measures of the eigenvalues, see [18]. The sine kernel, on the other hand, is a very detailed property which typically cannot be obtained from considerations of interacting particle systems. The Hamiltonian for GUE, however, is strictly convex and thus the Dyson's Brownian motion satisfies the logarithmic Sobolev inequality (LSI). It was noted in the derivation of the Navier-Stokes equations [12; 27] that the combination of the Guo-Papanicolaou-Varadhan [20] approach and LSI provides very detailed estimates on the dynamics.

The key observation of the present paper is that this method can also be used to estimate the approach to local equilibria so precisely that, after combining it with existing techniques from orthogonal polynomials, the Dyson sine kernel emerges. In pursuing this approach, we face two major obstacles: 1. Good estimate of the initial entropy, 2. Good understanding of the structure of local equilibria. It turns out that the initial entropy can be estimated using the explicitly formula for the transition kernel of the Dyson's Brownian motion (see [7] and [21]) provided strong inputs on the local semicircle law [14] and level repulsion [15] are available.

The structure of local equilibria, however, is much harder to analyze. Typically, the local equilibrium measures are finite volume Gibbs measures with short range interaction and the boundary effects can be easily dealt with in the high temperature phase. In the GUE case, the logarithmic potential does not even decay at large distance and the equilibrium measure can depend critically on the boundary conditions. The theory of orthogonal polynomials provides explicit formulae for the correlation functions of this highly correlated Gibbs measure. These formulae can be effectively analyzed if the external potential (or logarithm of the weight function in the terminology of the orthogonal polynomials) is very well understood. Fortunately, we have proved the local semicircle law up to scales of order $1 / N$ and the level repulsion, which can be used to control the boundary effects. By invoking the theorem of Levin and Lubinsky [23] and the method of Pastur and Shcherbina [26] we are led to the sine kernel.

It is easy to see that adding a Gaussian component of size much smaller than $N^{-1}$ to the original Wigner matrix would not move the eigenvalues sufficiently to change the local statistics. Our requirement that the Gaussian component is at least of size $N^{-3 / 4}$ comes from technical estimates 
to control the initial global entropy and it does not have any intrinsic meaning. The case that the variance is of order $N^{-1}$, however, is an intrinsic barrier which is difficult to cross. Nevertheless, we believe that our method may offer a possible strategy to prove the universality of sine kernel for general Wigner matrices.

After this manuscript had been completed, we found a different approach to prove the Dyson sine kernel [16], partly based on a contour integral representation for the two-point correlation function [7; 21]. Shortly after our manuscripts were completed, we learned that our main result was also obtained by Tao and $\mathrm{Vu}$ in [29] with a different method under no regularity conditions on the initial distribution $v$ provided the third moment of $v$ vanishes.

Although the results in this paper are weaker than those in [16] and [29], we believe that the method presented here has certain independent interest. Unlike [16] and [29], this approach does not use the contour integral representation of the two point correlation function. Hence, it may potentially have a broader applicability to other matrix ensembles for which such representation is not available.

Acknowledgements. We would like to thank the referees for suggesting several improvements of the presentation.

\section{Main theorem and conditions}

Fix $N \in \mathbb{N}$ and we consider a Hermitian matrix ensemble of $N \times N$ matrices $H=\left(h_{\ell k}\right)$ with the normalization

$$
h_{\ell k}=N^{-1 / 2} z_{\ell k}, \quad z_{\ell k}=x_{\ell k}+i y_{\ell k},
$$

where $x_{\ell k}, y_{\ell k}$ for $\ell<k$ are independent, identically distributed random variables with distribution $v=v^{(N)}$ that has zero expectation and variance $\frac{1}{2}$. The diagonal elements are real, i.e. $y_{\ell \ell}=0$ and and $x_{\ell \ell}$ are also i.i.d., independent from the off-diagonal ones with distribution $\widetilde{v}=\widetilde{v}^{(N)}$ that has zero expectation and variance one. The superscript indicating the $N$-dependence of $v, \widetilde{v}$ will be omitted.

We assume that the probability measures $v$ and $\widetilde{v}$ have a small Gaussian component of variance $N^{-3 / 4+\beta}$ where $\beta>0$ is some fixed positive number. More precisely, we assume there exist probability measures $v_{0}$ and $\widetilde{v}_{0}$ with zero expectation and variance $\frac{1}{2}$ and 1 , respectively, such that

$$
v=v_{s} * G_{s / \sqrt{2}}, \quad \widetilde{v}=\widetilde{v}_{s} * G_{s},
$$

where $G_{s}(x)=(2 \pi s)^{-1} \exp \left(-x^{2} / 2 s\right)$ is the Gaussian law with variance $s^{2}$ and $v_{s}$, $\widetilde{v}_{s}$ are the rescaling of the laws $v_{0}, \widetilde{v}_{0}$ to ensure that $v$ and $\widetilde{v}$ have variance $1 / 2$ and 1 ; i.e, explicitly

$$
v_{s}(\mathrm{~d} x)=\left(1-s^{2}\right)^{-1 / 2} v_{0}\left(\mathrm{~d} x\left(1-s^{2}\right)^{-1 / 2}\right), \quad \widetilde{v}_{s}(\mathrm{~d} x)=\left(1-s^{2}\right)^{-1 / 2} \widetilde{v}_{0}\left(\mathrm{~d} x\left(1-s^{2}\right)^{-1 / 2}\right) .
$$

This requirement is equivalent to considering random matrices of the form

$$
H=\left(1-s^{2}\right)^{1 / 2} \widehat{H}+s V,
$$

where $\widehat{H}$ is a Wigner matrix with single entry distribution $v_{0}$ and $\widetilde{v}_{0}$, and $V$ is a GUE matrix whose elements are centered Gaussian random variables with variance $1 / N$. 
Furthermore, we assume that $v$ is absolutely continuous with positive density functions $h(x)>0$, i.e. we can write it as $\mathrm{d} v(x)=h(x) \mathrm{d} x=\exp (-g(x)) \mathrm{d} x$ with some real function $g$. We assume the following conditions:

- The measure $\mathrm{d} v$ satisfies the logarithmic Sobolev inequality, i.e. there exists a constant $S$ such that

$$
\int_{\mathbb{R}} u \log u \mathrm{~d} v \leq S \int_{\mathbb{R}}|\nabla \sqrt{u}|^{2} \mathrm{~d} v
$$

holds for any density function $u>0$ with $\int u \mathrm{~d} v=1$.

- The Fourier transform of the functions $h$ and $h(\Delta g)$ satisfy the decay estimates

$$
|\widehat{h}(t, s)| \leq \frac{1}{\left[1+\omega\left(t^{2}+s^{2}\right)\right]^{9}}, \quad|\widehat{h \Delta g}(t, s)| \leq \frac{1}{\left[1+\widetilde{\omega}\left(t^{2}+s^{2}\right)\right]^{9}}
$$

with some constants $\omega, \tilde{\omega}>0$.

- There exists a $\delta_{0}>0$ such that for the distribution of the diagonal elements

$$
D_{0}:=\int_{\mathbb{R}} \exp \left[\delta_{0} x^{2}\right] \mathrm{d} \widetilde{v}(x)<\infty .
$$

Although the conditions are stated directly for the measures $v$ and $\widetilde{v}$, it is easy to see that it is sufficient to assume that $v_{0}$ satisfies (2.4) and (2.5) and $\widetilde{v}_{0}$ satisfies (2.6). We remark that (2.4) implies that (2.6) holds for $v$ instead of $\widetilde{v}$ as well (see [22]).

The eigenvalues of $H$ are denoted by $\lambda_{1}, \lambda_{2}, \ldots \lambda_{N}$. The law of the matrix ensemble induces a probability measure on the set of eigenvalues whose density function will be denoted by $p\left(\lambda_{1}, \lambda_{2}, \ldots, \lambda_{N}\right)$. The eigenvalues are considered unordered for the moment and thus $p$ is a symmetric function. For any $k=1,2, \ldots, N$, let

$$
p^{(k)}\left(\lambda_{1}, \lambda_{2}, \ldots \lambda_{k}\right):=\int_{\mathbb{R}^{N-k}} p\left(\lambda_{1}, \lambda_{2}, \ldots, \lambda_{N}\right) \mathrm{d} \lambda_{k+1} \ldots \mathrm{d} \lambda_{N}
$$

be the $k$-point correlation function of the eigenvalues. The $k=1$ point correlation function (density) is denoted by $\varrho(\lambda):=p^{(1)}(\lambda)$. With our normalization convention, the density $\varrho(\lambda)$ is supported in $[-2,2]$ and in the $N \rightarrow \infty$ limit it converges to the Wigner semicircle law given by the density

$$
\varrho_{s c}(x)=\frac{1}{2 \pi} \sqrt{4-x^{2}} 1_{[-2,2]}(x) .
$$

The main result of this paper is the following theorem:

Theorem 2.1. Fix arbitrary positive constants $\beta>0$ and $\kappa>0$. Consider the Wigner matrix ensemble with a Gaussian convolution of variance $s^{2}=N^{-3 / 4+\beta}$ given by (2.3) and assume (2.4)-(2.6). Let $p^{(2)}$ be the two point correlation function of the eigenvalues of this ensemble. Let $\left|E_{0}\right|<2-\kappa$ and

$$
O(a, b)=g(a-b) h\left(\frac{a+b}{2}\right)
$$


with $g, h$ smooth and compactly supported functions such that $h \geq 0$ and $\int h=1$. Then we have

$$
\begin{gathered}
\lim _{\delta \rightarrow 0 N \rightarrow \infty} \lim _{N \rightarrow} \frac{1}{2 \delta} \int_{E_{0}-\delta}^{E_{0}+\delta} \mathrm{d} E \iint \mathrm{d} a \mathrm{~d} b \\
O(a, b) \frac{1}{\rho_{s c}^{2}(E)} p^{(2)}\left(E+\frac{a}{\rho_{s c}(E) N}, E+\frac{b}{\rho_{s c}(E) N}\right) \\
=\int_{\mathbb{R}} g(u)\left[1-\left(\frac{\sin \pi u}{\pi u}\right)^{2}\right] \mathrm{d} u .
\end{gathered}
$$

The factor $g$ in the observable (2.8) tests the eigenvalue differences. The factor $h$, that disappears in the right hand side of (2.9), is only a normalization factor. Thus the special form of observable (2.8) directly exhibits the fact that the local statistics is translation invariant.

Conventions. All integrations with unspecified domains are on $\mathbb{R}$. We will use the letters $C$ and $c$ to denote general constants whose precise values are irrelevant and they may change from line to line. These constants may depend on the constants in (2.4)-(2.6).

\subsection{Outline of the proof}

Our approach has three main ingredients. In the first step, we use the entropy method from hydrodynamical limits to establish a local equilibrium of the eigenvalues in a window of size $N^{-1+\varepsilon}$ (with some small $\varepsilon>0$ ), i.e. window that typically contains $n=N^{\varepsilon}$ eigenvalues. This local equilibrium is subject to an external potential generated by all other eigenvalues. In the second step we then prove that the density of this equilibrium measure is locally constant by using methods from orthogonal polynomials. Finally, in the third step, we employ a recent result [23] to deduce the sine-kernel. We now describe each step in more details.

Step 1 .

We generate the Wigner matrix with a small Gaussian component by running a matrix-valued Ornstein-Uhlenbeck process (3.1) for a short time of order $t \sim N^{-\zeta}, \zeta>0$. This generates a stochastic process for the eigenvalues which can be described as Ornstein-Uhlenbeck processes for the individual eigenvalues with a strong interaction (3.10).

This process is the celebrated Dyson's Brownian motion (DBM) [11] and the equilibrium measure is the GUE distribution of eigenvalues. The transition kernel can be computed explicitly (5.12) and it contains the determinantal structure of the joint probability density of the GUE eigenvalues that is responsible for the sine-kernel. This kernel was analyzed by Johansson [21] assuming that the time $t$ is of order one, which is the same order as the relaxation time to equilibrium for the Dyson's Brownian motions. The sine-kernel, however, is a local statistics, and local equilibrium can be reached within a much shorter time scale. To implement this idea, we first control the global entropy on time scale $N^{-1}$ by $N^{1+\alpha}$, with $\alpha>1 / 4$ (Section 5.2).

More precisely, recall that the entropy of $f \mu$ with respect to a probability measure $\mu$ is given by

$$
S(f)=S_{\mu}(f):=S(f \mu \mid \mu)=\int f(\log f) \mathrm{d} \mu .
$$


In our application, the measure $\mu$ is the Gibbs measure for the equilibrium distribution of the (ordered) eigenvalues of the GUE, given by the Hamiltonian

$$
\mathscr{H}(\boldsymbol{\lambda})=N\left[\sum_{i=1}^{N} \frac{\lambda_{i}^{2}}{2}-\frac{2}{N} \sum_{i<j} \log \left|\lambda_{j}-\lambda_{i}\right|\right] .
$$

If $f_{t}$ denotes the joint probability density of the eigenvalues at the time $t$ with respect to $\mu$, then the evolution of $f_{t}$ is given by the equation

$$
\partial_{t} f_{t}=L f_{t}
$$

where the generator $L$ is defined via the Dirichlet form

$$
D(g)=\int g(-L) g \mathrm{~d} \mu=\frac{1}{2 N} \sum_{j=1}^{N} \int\left(\nabla_{\lambda_{j}} g\right)^{2} \mathrm{~d} \mu .
$$

The evolution of the entropy is given by the equation

$$
\partial_{t} S\left(f_{t}\right)=-D\left(\sqrt{f_{t}}\right)
$$

The key initial entropy estimate is the inequality that

$$
S_{\mu}\left(f_{s}\right):=S\left(f_{s} \mu \mid \mu\right) \leq C_{\alpha} N^{1+\alpha}, \quad s=1 / N
$$

for any $\alpha>\frac{1}{4}$ and for sufficiently large $N$. The proof of this estimate uses the explicit formula for the transition kernel of (2.11) and several inputs from our previous papers [13; $14 ; 15]$ on the local semicircle law and on the level repulsion for general Wigner matrices. We need to strengthen some of these inputs; the new result will be presented in Section 4 with proofs deferred to Appendix A, Appendix B and Appendix C.

It is natural to think of each eigenvalue as a particle and we will use the language of interacting particle systems. We remark that the entropy per particle is typically of order one in the interacting particle systems. But in our setting, due to the factor $N$ in front of the Hamiltonian (2.10), the typical size of entropy per particle is of order $N$. Thus for a system bearing little relation to the equilibrium measure $\mu$, we expect the total entropy to be $O\left(N^{2}\right)$. So the bound (2.12) already contains nontrivial information. However, we believe that one should be able to improve this bound to $\alpha \sim 0$ and the additional $\alpha>1 / 4$ power in (2.12) is only for technical reasons. This is the main reason why our final result holds only for a Gaussian convolution with variance larger than $N^{-3 / 4}$. The additional $N^{\alpha}$ factor originates from Lemma 5.3 where we approximate the Vandermonde determinant appearing in the transition kernel by estimating the fluctuations around the local semicircle law. We will explain the origin of $\alpha>1 / 4$ in the beginning of Appendix $D$ where the proof of Lemma 5.3 is given.

From the initial entropy estimate, it follows that the time integration of the Dirichlet form is bounded by the initial entropy. For the DBM, due to convexity of the Hamiltonian of the equilibrium measure $\mu$, the Dirichlet form is actually decreasing. Thus for $t=\tau N^{-1}$ with some $\tau \geq 2$ we have

$$
D\left(\sqrt{f}_{t}\right) \leq 2 S\left(f_{N^{-1}}\right) t^{-1} \leq C N^{2+\alpha} \tau^{-1} .
$$

The last estimate says that the Dirichlet form per particle is bounded by $N^{1+\alpha} \tau^{-1}$. So if we take an interval of $n$ particles (with coordinates given by $\mathbf{x}=\left(x_{1}, \ldots, x_{n}\right)$ ), then on average the total 
Dirichlet form of these particles is bounded by $n N^{1+\alpha} \tau^{-1}$. We will choose $n=N^{\varepsilon}$ with some very small $\varepsilon>0$. As always in the hydrodynamical limit approach, we consider the probability law of these $n$ particles given that all other particles (denoted by $\mathbf{y}$ ) are fixed. Denote by $\mu_{\mathbf{y}}(\mathrm{d} \mathbf{x})$ the equilibrium measure of $\mathbf{x}$ given that the coordinates of the other $N-n$ particles $\mathbf{y}$ are fixed. Let $f_{\mathbf{y}, t}$ be the conditional density of $f_{t}$ w.r.t. $\mu_{\mathbf{y}}(\mathrm{d} \mathbf{x})$ with $\mathbf{y}$ given. The Hamiltonian of the measure $\mu_{\mathbf{y}}(\mathrm{d} \mathbf{x})$ is given by

$$
\mathscr{H}_{\mathbf{y}}(\mathbf{x})=N\left[\sum_{i=1}^{n} \frac{1}{2} x_{i}^{2}-\frac{2}{N} \sum_{1 \leq i<j \leq n} \log \left|x_{j}-x_{i}\right|-\frac{2}{N} \sum_{k} \sum_{i=1}^{n} \log \left|x_{i}-y_{k}\right|\right]
$$

and it satisfies the convexity estimate

$$
\text { Hess } \mathscr{H}_{\mathbf{y}}(\mathbf{x}) \geq \sum_{k}\left|x-y_{k}\right|^{-2} \text {. }
$$

If $\mathbf{y}$ are regularly distributed, we have the convexity bound

$$
\text { Hess } \mathscr{H}_{\mathbf{y}}(\mathbf{x}) \geq \frac{c N^{2}}{n^{2}}
$$

This implies the logarithmic Sobolev inequality

$$
S_{\mu_{\mathbf{y}}}\left(f_{\mathbf{y}}\right) \leq C n^{2} N^{-1} D_{\mathbf{y}}\left(\sqrt{f_{\mathbf{y}}}\right) \leq C n^{6} N^{\alpha} \tau^{-1},
$$

where in the last estimate some additional $n$-factors were needed to convert the local Dirichlet form estimate per particle on average to an estimate that holds for a typical particle. Thus we obtain

$$
\left[\int\left|f_{\mathbf{y}}-1\right| \mathrm{d} \mu_{\mathbf{y}}\right]^{2} \leq S_{\mu_{\mathbf{y}}}\left(f_{\mathbf{y}}\right) \leq C n^{6} N^{\alpha} \tau^{-1} \leq n^{-4} \ll 1,
$$

provided we choose $t=N^{-1} \tau=N^{\beta-1}$ with $\beta \geq 10 \varepsilon+\alpha$ (Section 6 ). The last inequality asserts that the two measures $f_{\mathrm{y}} \mu_{\mathrm{y}}$ and $\mu_{\mathrm{y}}$ are almost the same and thus we only need to establish the sine kernel for the measure $\mu_{\mathbf{y}}$. At this point, we remark that this argument is valid only if $\mathbf{y}$ is regularly distributed in a certain sense which we will call good configurations (Definition 4.1). Precise estimates on the local semicircle law can be used to show that most external configurations are good. Although the rigorous treatment of the good configurations and estimates on the bad configurations occupy a large part of this paper, it is of technical nature and we deferred the proofs of several steps to the appendices.

Step 2.

In Sections 8, 9 and 10, we refine the precision on the local density and prove that the density is essentially constant pointwise. Direct probabilistic arguments to establish the local semicircle law in [15] rely on the law of large numbers and they give information on the density on scales of much larger than $N^{-1}$, i.e. on scales that contain many eigenvalues. The local equilibrium is reached in a window of size $n / N$ and within this window, we can conclude that the local semicircle law holds on scales of size $n^{\gamma} / N$ with an arbitrary small $\gamma>0$. However, this still does not control the density pointwise. To get this information, we need to use orthogonal polynomials. 
The density in local equilibrium can be expressed in terms of sum of squares of orthogonal polynomials $p_{1}(x), p_{2}(x), \ldots$ with respect to the weight function $\exp \left(-n U_{\mathbf{y}}(x)\right)$ generated by the external configuration $\mathbf{y}$ (see Section 8 for precise definitions). To get a pointwise bound from the appropriate bound on average, we need only to control the derivative of the density, that, in particular, can be expressed in terms of derivatives of the orthogonal polynomials $p_{k}$. Using integration by parts and orthogonality properties of $p_{k}$, it is possible to control the $L^{2}$ norm of $p_{k}^{\prime}$ in terms of the $L^{2}$ norm of $p_{k}(x) U_{\mathbf{y}}^{\prime}(x)$. Although the derivative of the potential is singular, $\left\|p_{k} U_{\mathbf{y}}^{\prime}\right\|_{2}$ can be estimated by a Schwarz inequality at the expense of treating higher $L^{p}$ norms of $p_{k}$ (Lemma 8.1). In this content, we will exploit the fact that we are dealing with polynomials by using the Nikolskii inequality which estimates higher $L^{p}$ norms in terms of lower ones at the expense of a constant depending on the degree. To avoid a very large constant in the Nikolskii inequality, in Section 7 we first cutoff the external potential and thus we reduce the degree of the weight function.

We remark that our approach of using orthogonal polynomials to control the density pointwise was motivated by the work of Pastur and Shcherbina [26], where they proved sine-kernel for unitary invariant matrix ensembles with a three times differentiable potential function on the real line. In our case, however, the potential is determined by the external points and it is logarithmically divergent near the edges of the window.

Step 3.

Finally, in Section 11, we complete the proof of the sine-kernel by applying the main theorem of [23]. This result establishes the sine-kernel for orthogonal polynomials with respect to an $n$ dependent sequence of weight functions under general conditions. The most serious condition to verify is that the density is essentially constant pointwise - the main result we have achieved in the Step 2 above. We also need to identify the support of the equilibrium measure which will be done in Appendix F.

We remark that, alternatively, it is possible to complete the third step along the lines of the argument of [26] without using [23]. Using explicit formulae from orthogonal polynomials and the pointwise control on the density and on its derivative, it is possible to prove that the local two-point correlation function $p_{n}^{(2)}(x, y)$ is translation invariant as $n \rightarrow \infty$. After having established the translation invariance of $p^{(2)}$, it is easy to derive an equation for its Fourier transform and obtain the sine-kernel as the unique solution of this equation. We will not pursue this alternative direction in this paper.

\section{Dyson's Brownian motion}

\subsection{Ornstein-Uhlenbeck process}

We can generate our matrix $H(2.3)$ from a stochastic process with initial condition $\widehat{H}$. Consider the following matrix valued stochastic differential equation

$$
\mathrm{d} H_{t}=\frac{1}{\sqrt{N}} \mathrm{~d} \boldsymbol{\beta}_{t}-\frac{1}{2} H_{t} \mathrm{~d} t
$$

where $\boldsymbol{\beta}_{t}$ is a hermitian matrix-valued stochastic process whose diagonal matrix elements are standard real Brownian motions and whose off-diagonal matrix elements are standard complex Brownian motions. 
For completeness we describe this matrix valued Ornstein-Uhlenbeck process more precisely. The rescaled matrix elements $z_{i j}=N^{1 / 2} h_{i j}$ evolve according to the complex Ornstein-Uhlenbeck process

$$
\mathrm{d} z_{i j}=\mathrm{d} \beta_{i j}-\frac{1}{2} z_{i j} \mathrm{~d} t, \quad i, j=1,2, \ldots N .
$$

For $i \neq j, \beta=\beta_{i j}$ is a complex Brownian motion with variance one. The real and imaginary parts of $z=x+i y$ satisfy

$$
\mathrm{d} x=\frac{1}{\sqrt{2}} \mathrm{~d} \beta_{x}-\frac{1}{2} x \mathrm{~d} t, \quad \mathrm{~d} y=\frac{1}{\sqrt{2}} \mathrm{~d} \beta_{y}-\frac{1}{2} y \mathrm{~d} t
$$

with $\beta=\frac{1}{\sqrt{2}}\left(\beta_{x}+i \beta_{y}\right)$ and where $\beta_{x}, \beta_{y}$ are independent standard real Brownian motions. For the diagonal elements $i=j$ in (3.2), $\beta_{i i}$ is a standard real Brownian motion with variance 1 .

To ensure $z_{i j}=\bar{z}_{j i}$, for $i<j$ we choose $\beta_{i j}$ to be independent complex Brownian motion with $\mathbb{E}\left|\beta_{i j}\right|^{2}=1$, we set $\beta_{j i}:=\bar{\beta}_{i j}$ and we let $\beta_{i i}$ to be a real Brownian motion with $\mathbb{E} \beta_{i i}^{2}=1$. Then

$$
\left(\mathrm{d} z_{i k}\right)\left(\mathrm{d} z_{\ell j}\right)=\left(\mathrm{d} \beta_{i k}\right)\left(\mathrm{d} \bar{\beta}_{j \ell}\right)=\delta_{i j} \delta_{k \ell} \mathrm{d} t
$$

We note that $\mathrm{d} \operatorname{Tr} H^{2}=0$, thus

$$
\operatorname{Tr} H^{2}=N
$$

remains constant for all time.

If the initial condition of (3.1) is distributed according to the law of $\widehat{H}$, then the solution of (3.1) is clearly

$$
H_{t}=e^{-t / 2} \widehat{H}+\left(1-e^{-t}\right)^{1 / 2} V
$$

where $V$ is a standard GUE matrix (with matrix elements having variance $1 / N$ ) that is independent of $\widehat{H}$. With the choice of $t$ satisfying $\left(1-e^{-t}\right)=s^{2}=N^{-3 / 4+\beta}$, i.e. $t=-\log \left(1-N^{-3 / 4+\beta}\right) \approx$ $N^{-3 / 4+\beta}$, we see that $H$ given in (2.3) has the same law as $H_{t}$.

\subsection{Joint probability distribution of the eigenvalues}

We will now analyze the eigenvalue distribution of $H_{t}$. Let $\lambda(t)=\left(\lambda_{1}(t), \lambda_{2}(t), \ldots, \lambda_{N}(t)\right) \in \mathbb{R}^{N}$ denote the eigenvalues of $H_{t}$. As $t \rightarrow \infty$, the Ornstein-Uhlenbeck process (3.1) converges to the standard GUE. The joint distribution of the GUE eigenvalues is given by the following measure $\tilde{\mu}$ on $\mathbb{R}^{N}$

$$
\tilde{\mu}=\tilde{\mu}(\mathrm{d} \lambda)=\frac{e^{-\mathscr{H}(\boldsymbol{\lambda})}}{Z} \mathrm{~d} \boldsymbol{\lambda}, \quad \mathscr{H}(\boldsymbol{\lambda})=N\left[\sum_{i=1}^{N} \frac{\lambda_{i}^{2}}{2}-\frac{2}{N} \sum_{i<j} \log \left|\lambda_{j}-\lambda_{i}\right|\right] .
$$

The measure $\tilde{\mu}$ has a density with respect to Lebesgue measure given by

$$
\widetilde{u}(\boldsymbol{\lambda})=\frac{N^{N^{2} / 2}}{(2 \pi)^{N / 2} \prod_{j=1}^{N} j !} \exp \left[-\frac{N}{2} \sum_{j=1}^{N} \lambda_{j}^{2}\right] \Delta_{N}(\boldsymbol{\lambda})^{2}, \quad \tilde{\mu}(\mathrm{d} \lambda)=\widetilde{u}(\boldsymbol{\lambda}) \mathrm{d} \boldsymbol{\lambda},
$$

where $\Delta_{N}(\lambda)=\prod_{i<j}\left(\lambda_{i}-\lambda_{j}\right)$. This is the joint probability distribution of the eigenvalues of the standard GUE ensemble normalized in such a way that the matrix elements have variance $1 / N$ (see, 
e.g. [25]). With this normalization convention, the bulk of the one point function (density) is supported in $[-2,2]$ and in the $N \rightarrow \infty$ limit it converges to the Wigner semicircle law (2.7).

For any finite time $t<\infty$ we will represent the joint probability density of the eigenvalues of $H_{t}$ as $f_{t}(\boldsymbol{\lambda}) \widetilde{u}(\boldsymbol{\lambda})$, with $\lim _{t \rightarrow \infty} f_{t}(\boldsymbol{\lambda})=1$. In particular, we write the joint distribution of the eigenvalues of the initial Wigner matrix $\widehat{H}$ as $f_{0}(\boldsymbol{\lambda}) \tilde{\mu}(\mathrm{d} \lambda)=f_{0}(\boldsymbol{\lambda}) \widetilde{u}(\boldsymbol{\lambda}) \mathrm{d} \boldsymbol{\lambda}$.

\subsection{The generator of Dyson's Brownian motion}

The Ornstein-Uhlenbeck process (3.1) induces a stochastic process for the eigenvalues.

Let $L$ be the generator given by

$$
L=\sum_{i=1}^{N} \frac{1}{2 N} \partial_{i}^{2}+\sum_{i=1}^{N}\left(-\frac{1}{2} \lambda_{i}+\frac{1}{N} \sum_{j \neq i} \frac{1}{\lambda_{i}-\lambda_{j}}\right) \partial_{i}
$$

acting on $L^{2}(\widetilde{\mu})$ and let

$$
D(f)=-\int f L f \mathrm{~d} \tilde{\mu}=\sum_{j=1}^{N} \frac{1}{2 N} \int\left(\partial_{j} f\right)^{2} \mathrm{~d} \tilde{\mu}
$$

be the corresponding Dirichlet form, where $\partial_{j}=\partial_{\lambda_{j}}$. Clearly $\tilde{\mu}$ is an invariant measure for the dynamics generated by $L$.

Let the distribution of the eigenvalues of the Wigner ensemble be given by $f_{0}(\lambda) \widetilde{\mu}(\mathrm{d} \lambda)$. We will evolve this distribution by the dynamics given by $L$ :

$$
\partial_{t} f_{t}=L f_{t}
$$

The corresponding stochastic differential equation for the eigenvalues $\lambda(t)$ is now given by (see, e.g. Section 12.1 of [19])

$$
\mathrm{d} \lambda_{i}=\frac{\mathrm{d} B_{i}}{\sqrt{N}}+\left[-\frac{1}{2} \lambda_{i}+\frac{1}{N} \sum_{j \neq i} \frac{1}{\lambda_{i}-\lambda_{j}}\right] \mathrm{d} t, \quad 1 \leq i \leq N,
$$

where $\left\{B_{i}: 1 \leq i \leq N\right\}$ is a collection of independent Brownian motions and with initial condition $\lambda(0)$ that is distributed according to the probability density $f_{0}(\lambda) \widetilde{\mu}(\mathrm{d} \lambda)$.

We remark that $\widetilde{u}(\boldsymbol{\lambda})$ and $f_{t}(\boldsymbol{\lambda})$ are symmetric functions of the variables $\lambda_{j}$ and $\widetilde{u}$ vanishes whenever two points coincide. By the level repulsion we also know that $f_{0}(\lambda) \widetilde{u}(\boldsymbol{\lambda})$ vanishes whenever $\lambda_{j}=\lambda_{k}$ for some $j \neq k$. We can label the eigenvalues according to their ordering, $\lambda_{1}<\lambda_{2}<\ldots<\lambda_{N}$, i.e. one can consider the configuration space

$$
\Xi^{(N)}:=\left\{\lambda=\left(\lambda_{1}, \lambda_{2}, \ldots, \lambda_{N}\right): \lambda_{1}<\lambda_{2}<\ldots<\lambda_{N}\right\} \subset \mathbb{R}^{N}
$$

instead of the whole $\mathbb{R}^{N}$. With an initial point in $\Xi^{(N)}$, the equation 3.10 ) has a unique solution and the trajectories do not cross each other, i.e. the ordering of eigenvalues is preserved under the time evolution and thus the dynamics generated by $L$ can be restricted to $\Xi^{(N)}$; see, e.g. Section 12.1 of [19]. The main reason is that near a coalescence point $\lambda_{i}=\lambda_{j}, i>j$, the generator is

$$
\frac{1}{N}\left[\frac{1}{2} \partial_{\lambda_{i}}^{2}+\frac{1}{2} \partial_{\lambda_{j}}^{2}+\frac{1}{\lambda_{i}-\lambda_{j}}\left(\partial_{\lambda_{j}}-\partial_{\lambda_{i}}\right)\right]=\frac{1}{2 N}\left[\frac{1}{2} \partial_{a}^{2}+\frac{1}{2} \partial_{b}^{2}+\frac{1}{b} \partial_{b}\right]
$$


with $a=\frac{1}{2}\left(\lambda_{i}+\lambda_{j}\right), b=\frac{1}{2}\left(\lambda_{i}-\lambda_{j}\right)$. The constant 1 in front of the drift term is critical for the Bessel process $\frac{1}{2} \partial_{b}^{2}+\frac{1}{b} \partial_{b}$ not to reach the boundary point $b=0$.

Note that the symmetric density function $\widetilde{u}(\boldsymbol{\lambda})$ defined on $\mathbb{R}^{N}$ can be restricted to $\Xi^{(N)}$ as

$$
u(\boldsymbol{\lambda})=N ! \widetilde{u}(\boldsymbol{\lambda}) \mathbf{1}\left(\boldsymbol{\lambda} \in \Xi^{(N)}\right) .
$$

The density function of the ordered eigenvalues is thus $f_{t}(\boldsymbol{\lambda}) u(\boldsymbol{\lambda})$ on $\Xi^{(N)}$. Throughout this paper, with the exception of Section 5.2 , we work on the space $\Xi^{(N)}$, i.e., the equilibrium measure $\mu(\mathrm{d} \lambda)=$ $u(\lambda) \mathrm{d} \boldsymbol{\lambda}$ with density $u(\boldsymbol{\lambda})$ and the density function $f_{t}(\boldsymbol{\lambda})$ will be considered restricted to $\Xi^{(N)}$.

\section{Good global configurations}

Several estimates in this paper will rely on the fact that the number of eigenvalues $\mathscr{N}_{I}$ in intervals $I$ with length much larger than $1 / N$ is given by the semicircle law [15]. In this section we define the set of good global configurations, i.e. the event that the semicircle law holds on all subintervals in addition to a few other typical properties.

Let

$$
\omega(\mathrm{d} x)=\frac{1}{N} \sum_{j=1}^{N} \delta\left(x-\lambda_{j}\right)
$$

be the empirical density of the eigenvalues. For an interval $I=[a, b]$ we introduce the notation

$$
\mathscr{N}_{I}=\mathscr{N}[a ; b]=N \int_{a}^{b} \omega(\mathrm{d} x)
$$

for the number of eigenvalues in $I$. For the interval $[E-\eta / 2, E+\eta / 2]$ of length $\eta$ and centered at $E$ we will also use the notation

$$
\mathscr{N}_{\eta}(E):=\mathscr{N}[E-\eta / 2 ; E+\eta / 2]
$$

Let

$$
\omega_{\eta}(x):=\left(\theta_{\eta} * \omega\right)(x), \quad \text { with } \quad \theta_{\eta}(x)=\frac{1}{\pi} \frac{\eta}{x^{2}+\eta^{2}}
$$

be the empirical density smoothed out on scale $\eta$. Furthermore, let

$$
m(z)=\frac{1}{N} \sum_{j=1}^{N} \frac{1}{\lambda_{j}-z}=\int_{\mathbb{R}} \frac{\omega(\mathrm{d} x)}{x-z}
$$

be the Stieltjes transform of the empirical eigenvalue distribution and

$$
m_{s c}(z)=\int_{\mathbb{R}} \frac{\varrho_{s c}(x)}{x-z} \mathrm{~d} x=-\frac{z}{2}+\sqrt{\frac{z^{2}}{4}-1}
$$

be the Stieljes transform of the semicircle law. The square root here is defined as the analytic extension (away from the branch cut $[-2,2]$ ) of the positive square root on large positive numbers. Clearly $\omega_{y}(x)=\pi^{-1} \operatorname{Im} m(x+i y)$ for $y>0$.

We will need an improved version of Theorem 4.1 from [15] that is also applicable near the spectral edges. The proof of the following theorem is given in Appendix A. 
Theorem 4.1. Assume that the Wigner matrix ensemble satisfies conditions (2.4)-(2.6) and assume that $y$ is such that $(\log N)^{4} / N \leq|y| \leq 1$.

(i) For any $q \geq 1$ we have

$$
\begin{gathered}
\mathbb{E}|m(x+i y)|^{q} \leq C_{q} \\
\mathbb{E}\left[\omega_{y}(x)\right]^{q} \leq C_{q}
\end{gathered}
$$

where $C_{q}$ is independent of $x$ and $y$.

(ii) Assume that $|x| \leq K$ for some $K>0$. Then there exists $c>0$ such that

$$
\mathbb{P}\left(\left|m(x+i y)-m_{s c}(x+i y)\right| \geq \delta\right) \leq C e^{-c \delta \sqrt{N|y||2-| x||}}
$$

for all $\delta>0$ small enough and all $N$ large enough (independently of $\delta$ ). Consequently, we have

$$
\mathbb{E}|m(x+i y)-\mathbb{E} m(x+i y)|^{q} \leq \frac{C_{q}}{(N|y||2-| x||)^{q / 2}}+C_{q} \mathbf{1}\left(N|y||2-| x|| \leq(\log N)^{4}\right)
$$

with some q-dependent constant $C_{q}$. Moreover,

$$
\left|\mathbb{E} m(x+i y)-m_{s c}(x+i y)\right| \leq \frac{C}{N|y|^{3 / 2}|2-| x||^{1 / 2}}
$$

for all $N$ large enough (independently of $x, y$ ).

(iii) Assuming $|x| \leq K$ and that $\sqrt{N|y||2-| x||} \geq(\log N)^{2}$ we also have

$$
\left|\mathbb{E} m(x+i y)-m_{s c}(x+i y)\right| \leq \frac{C}{\left.N|y||2-| x\right|^{3 / 2}} .
$$

As a corollary to Theorem 4.1, the semicircle law for the density of states holds locally on very short scales. The next proposition can be proved, starting from Theorem 4.1, exactly as Eq. (4.3) was shown in [13].

Proposition 4.1. Assuming (2.4)-(2.6), for any sufficiently small $\delta$ and for any $\eta^{*}$ with

$$
C \delta^{-2}(\log N)^{4} / N \leq \eta^{*} \leq C^{-1} \min \{\kappa, \delta \sqrt{\kappa}\}
$$

(with a sufficiently large constant $C$ ) we have

$$
\mathbb{P}\left\{\sup _{E \in[-2+\kappa, 2-\kappa]}\left|\frac{\mathscr{N}_{\eta^{*}}(E)}{2 N \eta^{*}}-\varrho_{s c}(E)\right| \geq \delta\right\} \leq C e^{-c \delta^{2} \sqrt{N \eta^{*} \kappa}} .
$$

We also need an estimate directly on the number of eigenvalues in a certain interval, but this will be needed only away from the spectral edge. The following two results estimate the deviation of the normalized empirical counting function $\frac{1}{N} \mathscr{N}[-\infty, E]=\frac{1}{N} \#\left\{\lambda_{j} \leq E\right\}$ and its expectation

$$
\mathfrak{N}(E):=\frac{1}{N} \mathbb{E} \mathscr{N}[-\infty, E]
$$

from the distribution function of the semicircle law, defined as

$$
\mathfrak{N}_{s c}(E):=\int_{-\infty}^{E} \varrho_{s c}(x) \mathrm{d} x .
$$


Proposition 4.2. Assume that the Wigner matrix ensemble satisfies conditions (2.4)-(2.6). Let $\kappa>0$ be fixed. For any $0<\delta<1$ and $|E| \leq 2-\kappa$, we have

$$
\mathbb{P}\left\{\left|\frac{\mathscr{N}[-\infty, E]}{N}-\mathfrak{N}_{s c}(E)\right| \geq \delta\right\} \leq C e^{-c \delta \sqrt{N}}
$$

with $\kappa$-dependent constants. Moreover, there exists a constant $C>0$ such that

$$
\int_{-\infty}^{\infty}\left|\mathfrak{N}(E)-\mathfrak{N}_{s c}(E)\right| \mathrm{d} E \leq \frac{C}{N^{6 / 7}}
$$

The proof of this proposition will be given in Appendix B.

Next we define the good global configurations; the idea is that good global configurations are configurations for which the semicircle law holds up to scales of the order $(\log N)^{4} / N$ (and so that some more technical conditions are also satisfied). By Proposition 4.1 and Proposition 4.2, we will see that set of these configurations have, asymptotically, a full measure. As a consequence, we will be able to neglect all configurations that are not good.

Let

$$
n:=2\left[N^{\varepsilon} / 2\right]+1, \quad \eta_{m}^{*}=2^{m} n^{\gamma} N^{-1}, \quad \delta_{m}=2^{-m / 4} n^{-\gamma / 6}
$$

with some small constants $0<\varepsilon, \gamma \leq \frac{1}{10}$ and $m=0,1,2, \ldots, \log N$. Here $[x]$ denotes the integer part of $x \in \mathbb{R}$. Note that within this range of $m$ 's, $C \delta_{m}^{-2}(\log N)^{4} / N \leq \eta_{m}^{*} \leq \kappa^{3 / 4} \delta_{m}^{1 / 2}$ is satisfied if $\varepsilon, \gamma$ are sufficiently small. Let

$$
\Omega^{(m)}:=\left\{\sup _{E \in[-2+\kappa / 2,2-\kappa / 2]}\left|\frac{\mathscr{N}_{m}^{*}(E)}{N \eta_{m}^{*}}-\varrho_{s c}(E)\right| \leq \frac{1}{\left(N \eta_{m}^{*}\right)^{1 / 4}} n^{\gamma / 12}\right\}
$$

then we have

$$
\mathbb{P}\left(\Omega^{(m)}\right) \geq 1-C e^{-c n^{r / 6}}
$$

with respect to any Wigner ensemble. This gives rise to the following definition.

Definition 4.1. Let $\eta_{m}^{*}=2^{m} n^{\gamma} N^{-1}$ with some small constant $\gamma>0, m=0,1,2, \ldots \log N$, and let $K$ be a fixed big constant. The event

$$
\Omega:=\bigcap_{m=0}^{\log N} \Omega^{(m)} \cap\left\{\left|\frac{\mathscr{N}[-\infty, 0]}{N / 2}-1\right| \leq n^{-\gamma / 6}\right\} \cap\left\{\sup _{E} \mathscr{N}_{\eta_{0}^{*}}(E) \leq K N \eta_{0}^{*}\right\} \cap\{\mathscr{N}(-K, K)=N\}
$$

will be called the set of good global configurations.

Lemma 4.2. The probability of good global configurations satisfies

$$
\mathbb{P}(\Omega) \geq 1-C e^{-c n^{\gamma / 6}}
$$

with respect to any Wigner ensemble satisfying the conditions (2.4) and (2.5) 
Proof. The probability of $\Omega^{(m)}$ was estimated in (4.17). The probability of the second event in (4.18) can be estimated by (4.13) from Proposition 4.2 and from $\mathfrak{N}_{s c}(0)=1 / 2$. The third event is treated by the large deviation estimate on $\mathscr{N}_{I}$ for any interval $I$ with length $|I| \geq(\log N)^{2} / N$ (see Theorem 4.6 from [15]; note that there is a small error in the statement of this theorem, since the conditions $y \geq(\log N) / N$ and $|I| \geq(\log N) / N$ should actually be replaced by the stronger assumptions $y \geq(\log N)^{2} / N$ and $|I| \geq(\log N)^{2} / N$ which are used in its proof):

$$
\mathbb{P}\left\{\mathscr{N}_{I} \geq K N|I|\right\} \leq e^{-c \sqrt{K N|I|}} .
$$

The fourth event is a large deviation of the largest eigenvalue, see, e.g. Lemma 7.4. in [13].

In case of good configurations, the location of the eigenvalues are close to their equilibrium localition given by the semicircle law. The following lemma contains the precise statement and it will be proven in Appendix $\mathrm{C}$.

Lemma 4.3. Let $\lambda_{1}<\lambda_{2}<\ldots<\lambda_{N}$ denote the eigenvalues in increasing order and let $\kappa>0$. Then on the set $\Omega$ and if $N \geq N_{0}(\kappa)$, it holds that

$$
\left|\lambda_{a}-\mathfrak{N}_{s c}^{-1}\left(a N^{-1}\right)\right| \leq C \kappa^{-1 / 2} n^{-\gamma / 6}
$$

for any $N \kappa^{3 / 2} \leq a \leq N\left(1-\kappa^{3 / 2}\right)$ (recall the definition of $\mathfrak{N}_{s c}$ from (4.12)), and

$$
\left|N \varrho_{s c}\left(\lambda_{a}\right)\left(\lambda_{b}-\lambda_{a}\right)-(b-a)\right| \leq C \kappa^{-1 / 2}\left[n^{\gamma}|b-a|^{3 / 4}+N^{-1}|b-a|^{2}\right]
$$

for any $N \kappa^{3 / 2} \leq a<b \leq N\left(1-\kappa^{3 / 2}\right)$ and $|b-a| \leq C N n^{-\gamma / 6}$.

\subsection{Bound on the level repulsion and potential for good configurations}

Lemma 4.4. On the set $\Omega$ and with the choice $n$ given in (4.15), we have

$$
\frac{1}{N} \mathbb{E} \sum_{\ell=N \kappa^{3 / 2}}^{\left(1-\kappa^{3 / 2}\right) N} \sum_{j \neq \ell} \frac{\mathbf{1}_{\Omega}}{\left[N\left(\lambda_{j}-\lambda_{\ell}\right)\right]^{2}} \leq C n^{2 \gamma} .
$$

and

$$
\frac{1}{N} \mathbb{E} \sum_{\ell=N \kappa^{3 / 2}}^{\left(1-\kappa^{3 / 2}\right) N} \sum_{j \neq \ell} \frac{\mathbf{1}_{\Omega}}{N\left(\lambda_{\ell}-\lambda_{j}\right)} \leq C n^{2 \gamma}
$$

with respect to any Wigner ensemble satisfying the conditions (2.4) and (2.5)

Proof. First we partition the interval $[-2+\kappa, 2-\kappa]$ into subintervals

$$
I_{r}=\left[n^{\gamma} N^{-1}\left(r-\frac{1}{2}\right), n^{\gamma} N^{-1}\left(r+\frac{1}{2}\right)\right], \quad r \in \mathbb{Z},|r| \leq r_{1}:=(2-\kappa) N n^{-\gamma},
$$

that have already been used in the proof of Lemma 4.3. On the set $\Omega$ we have the bound

$$
\mathscr{N}\left(I_{r}\right) \leq K N\left|I_{r}\right| \leq C n^{\gamma}
$$


on the number of eigenvalues in each interval $I_{r}$. Moreover, the constraint $N \kappa^{3 / 2} \leq \ell \leq N\left(1-\kappa^{3 / 2}\right)$ implies, by (4.21), that $\left|\lambda_{\ell}\right| \leq 2-\kappa$ for sufficiently small $\kappa$, thus $\lambda_{\ell} \in I_{r}$ with $|r| \leq r_{1}$.

We estimate 4.23 as follows:

$$
\begin{aligned}
A & :=\frac{1}{N} \mathbb{E} \mathbf{1}_{\Omega} \sum_{j<\ell}^{*} \frac{1}{\left[N\left(\lambda_{j}-\lambda_{\ell}\right)\right]^{2}} \\
& =\frac{1}{N} \mathbb{E} \mathbf{1}_{\Omega} \sum_{j<\ell} \sum_{k \in \mathbb{Z}|r| \leq r_{1}} \frac{\mathbf{1}\left(\lambda_{\ell} \in I_{r}\right) \mathbf{1}\left(2^{k} \leq N\left|\lambda_{j}-\lambda_{\ell}\right| \leq 2^{k+1}\right)}{\left[N\left(\lambda_{j}-\lambda_{\ell}\right)\right]^{2}} \\
& \leq \frac{1}{N} \mathbb{E} \mathbf{1}_{\Omega} \sum_{|r| \leq r_{1}} \sum_{j<\ell} \sum_{k \in \mathbb{Z}} 2^{-2 k} \mathbf{1}\left\{\lambda_{\ell} \in I_{r}, 2^{k} \leq N\left|\lambda_{j}-\lambda_{\ell}\right| \leq 2^{k+1}\right\}
\end{aligned}
$$

where the star in the first summation indicates a restriction to $N \kappa^{3 / 2} \leq j<\ell \leq\left(1-\kappa^{3 / 2}\right) N$. By (4.26), for any fixed $r$, the summation over $\ell$ with $\lambda_{\ell} \in I_{r}$ contains at most $C n^{\gamma}$ elements. The summation over $j$ contains at most $C n^{\gamma}$ elements if $k<0$, since $\lambda_{\ell} \in I_{r}$ and $\left|\lambda_{j}-\lambda_{\ell}\right| \leq 2^{k+1} N^{-1} \leq$ $N^{-1}$ imply that $\lambda_{j} \in I_{r} \cup I_{r+1}$. If $k \geq 0$, then the $j$-summation has at most $C\left(2^{k}+n^{\gamma}\right)$ elements since in this case $\lambda_{j} \in \bigcup\left\{I_{s}:|s-r| \leq C \cdot 2^{k} n^{-\gamma}+1\right\}$. Thus we can continue the above estimate as

$$
\begin{aligned}
A \leq & \frac{C n^{2 \gamma}}{N} \sum_{k<0} \sum_{|r| \leq r_{1}} 2^{-2 k} \mathbb{P}\left\{\exists I \subset I_{r-1} \cup I_{r} \cup I_{r+1}:|I| \leq 2^{k+1} N^{-1}, \mathscr{N}_{I} \geq 2\right\} \\
& +\frac{C n^{\gamma}}{N} \sum_{k \geq 0} \sum_{|r| \leq r_{1}} 2^{-2 k}\left(n^{\gamma}+2^{k}\right) .
\end{aligned}
$$

The second sum is bounded by $\mathrm{Cn}^{3 \gamma}$. In the first sum, we use the level repulsion estimate by decomposing $I_{r-1} \cup I_{r} \cup I_{r+1}=\bigcup_{m} J_{m}$ into intervals of length $2^{k+2} N^{-1}$ that overlap at least by $2^{k+1} N^{-1}$, more precisely

$$
J_{m}=\left[n^{\gamma} N^{-1}\left(r-1-\frac{1}{2}\right)+2^{k+1} N^{-1}(m-1), n^{\gamma} N^{-1}\left(r-1-\frac{1}{2}\right)+2^{k+1} N^{-1}(m+1)\right],
$$

where $m=1,2, \ldots, 3 n^{\gamma} \cdot 2^{-k-1}$. Then

$$
\mathbb{P}\left\{\exists I \subset I_{r-1} \cup I_{r} \cup I_{r+1}:|I| \leq 2^{k+1} N^{-1}, \mathscr{N}_{I} \geq 2\right\} \leq \sum_{m=1}^{3 n^{\gamma} \cdot 2^{-k-1}} \mathbb{P}\left\{\mathscr{N}_{J_{m}} \geq 2\right\}
$$

Using the level repulsion estimate given in Theorem 3.4 of [15] (here the condition (2.5) is used) and the fact that $J_{m} \subset I_{r-1} \cup I_{r} \cup I_{r+1} \subset[-2+\kappa, 2-\kappa]$ since $|r| \leq r_{1}$, we have

$$
\mathbb{P}\left\{\mathscr{N}_{J_{m}} \geq 2\right\} \leq C\left(N\left|J_{m}\right|\right)^{4}
$$

and thus

$$
A \leq \frac{C n^{3 \gamma}}{N} \sum_{k=-\infty}^{-1} \sum_{|r| \leq r_{1}} 2^{-2 k} 2^{-k-1}\left(2^{k+2}\right)^{4} \leq C n^{2 \gamma} .
$$

and this completes the proof of 4.23). 
For the proof of (4.24), we note that it is sufficient to bound the event when $N\left|\lambda_{j}-\lambda_{\ell}\right| \geq 1$ after using (4.23). Inserting the partition (4.25), we get

$$
\begin{aligned}
\frac{1}{N} \mathbb{E} \mathbf{1}_{\Omega} \sum_{j<\ell}^{*} \frac{\mathbf{1}\left(N\left|\lambda_{\ell}-\lambda_{j}\right| \geq 1\right)}{N\left(\lambda_{\ell}-\lambda_{j}\right)} & =\frac{1}{N} \sum_{|r|,|s| \leq r_{0}} \mathbb{E} \mathbf{1}_{\Omega} \sum_{j<\ell} \frac{\mathbf{1}\left(\lambda_{j} \in I_{r}, \lambda_{\ell} \in I_{s}\right) \mathbf{1}\left(N\left|\lambda_{\ell}-\lambda_{j}\right| \geq 1\right)}{N\left(\lambda_{\ell}-\lambda_{j}\right)} \\
& \leq \frac{C}{N} \sum_{|r|,|s| \leq r_{0}} \mathbb{E} \mathbf{1}_{\Omega} \frac{\mathscr{N}_{I_{r} \mathscr{N}_{I_{s}}}}{n^{\gamma}[|s-r|-1]_{+}+1} \\
& \leq \frac{C n^{2 \gamma}}{N} \sum_{|r|,|s| \leq r_{0}} \frac{1}{n^{\gamma}[|s-r|-1]_{+}+1} \\
& \leq C n^{\gamma} \log N .
\end{aligned}
$$

Recalling the choice of $n$ completes the proof of Lemma 4.4 .

\section{Global entropy}

\subsection{Evolution of the entropy}

Recall the definition of the entropy of $f \mu$ with respect to $\mu$

$$
S_{\mu}(f):=S(f \mu \mid \mu)=\int f(\log f) \mathrm{d} \mu
$$

and let $f_{t}$ solve (3.9). Then the evolution of the entropy is given by the equation

$$
\partial_{t} S\left(f_{t}\right)=-D\left(\sqrt{f_{t}}\right)
$$

and thus using that $S\left(f_{t}\right)>0$ we have

$$
\int_{s}^{t} D\left(\sqrt{f_{u}}\right) \mathrm{d} u \leq S\left(f_{s}\right) .
$$

For dynamics with energy $\mathscr{H}$ and the convexity condition

$$
\operatorname{Hess}(\mathscr{H})=\nabla^{2} \mathscr{H} \geq \Lambda
$$

for some constant $\Lambda$, the following Bakry-Emery inequality [2] holds:

$$
\partial_{t} D\left(\sqrt{f_{t}}\right) \leq-\frac{\Lambda}{N} D\left(\sqrt{f_{t}}\right)
$$

(notice the additional $N$ factor due to the $N^{-1}$ in front of the second order term in the generator $L$, see (3.7)). This implies the logarithmic Sobolev inequality that for any probability density $g$, with respect to $\mu$,

$$
D(\sqrt{g})=-\int \sqrt{g} L \sqrt{g} \mathrm{~d} \mu \geq \frac{\Lambda}{N} S(g)
$$


In this case, the Dirichlet form is a decreasing function in time and we thus have for any $t>s$ that

$$
D\left(\sqrt{f}_{t}\right) \leq \frac{S\left(f_{s}\right)}{t-s}
$$

In our setting, we have

$$
\operatorname{Hess}(\mathscr{H})=\frac{\partial^{2} \mathscr{H}}{\partial \lambda_{i} \partial \lambda_{j}}=\delta_{i j}\left(N+\sum_{k \neq j} \frac{2}{\left(\lambda_{j}-\lambda_{k}\right)^{2}}\right)-\delta_{i \neq j} \frac{2}{\left(\lambda_{i}-\lambda_{j}\right)^{2}} \geq N \cdot \mathrm{Id}
$$

as a matrix inequality away from the singularities (see remark below how to treat the singular set). Thus we have

$$
\partial_{t} D\left(\sqrt{f}_{t}\right) \leq-D\left(\sqrt{f}_{t}\right)
$$

and by (5.3)

$$
\partial_{t} S\left(f_{t}\right) \leq-S\left(f_{t}\right)
$$

This tells us that $S\left(f_{t}\right)$ in (3.9) is exponential decaying as long as $t \gg 1$. But for any time $t \sim 1$ fixed, the entropy is still the same order as the initial one. Note that $t \sim 1$ is the case considered in Johasson's work [21].

Remark 5.1. The proof of (5.5) and the application of the Bakry-Emery condition in (5.6) requires further justification. Typically, Bakry-Emery condition is applied for Hamiltonians $\mathscr{H}$ defined on spaces without boundary. Although the Hamiltonian $\mathscr{H}(3.5)$ is defined on $\mathbb{R}^{N}$, it is however convex only away from any coalescence points $\lambda_{i}=\lambda_{j}$ for some $i \neq j$; the Hessian of the logarithmic terms has a Dirac delta singularity with the wrong (negative) sign whenever two particles overlap. In accordance with the convention that we work on the space $\Xi^{(N)}$ throughout the paper, we have to consider $\mathscr{H}$ restricted to $\Xi^{(N)}$, where it is convex, i.e. (5.5) holds, but we have to verify that the Bakry-Emery result still applies. We review the proof of Bakry and Emery and prove that the contribution of the boundary term is zero.

Recall that the invariant measure $\exp (-\mathscr{H}) \mathrm{d} \lambda$ and the dynamics $L=\frac{1}{2 N}[\Delta-(\nabla \mathscr{H}) \nabla]$ are restricted to $\Xi=\Xi^{(N)}$. With $h=\sqrt{f}$ we have

$$
\partial_{t} h^{2}=L h^{2}=2 h L h+\frac{1}{N}(\nabla h)^{2}, \quad \text { i.e. } \quad \partial_{t} h=L h+\frac{1}{2 N} h^{-1}(\nabla h)^{2} .
$$

Computing $\partial_{t} D\left(\sqrt{f_{t}}\right)$, we have

$$
\begin{aligned}
\partial_{t} \frac{1}{2 N} \int_{\Xi}(\nabla h)^{2} e^{-\mathscr{H}} \mathrm{d} \lambda & =\frac{1}{N} \int_{\Xi} \nabla h \nabla\left(L h+\frac{1}{2 N} h^{-1}(\nabla h)^{2}\right) e^{-\mathscr{H}} \mathrm{d} \lambda \\
& =\frac{1}{N} \int_{\Xi}\left[\nabla h L \nabla h-\frac{1}{2 N} \nabla h\left(\nabla^{2} \mathscr{H}\right) \nabla h+\frac{1}{2 N}(\nabla h) \nabla\left[h^{-1}(\nabla h)^{2}\right]\right] e^{-\mathscr{H}} \mathrm{d} \lambda \\
& =\frac{1}{2 N^{2}} \int_{\Xi}\left[-\nabla h\left(\nabla^{2} \mathscr{H}\right) \nabla h-\sum_{i, j}\left(\partial_{i j}^{2} h-\frac{\partial_{i} h \partial_{j} h}{h}\right)^{2}\right] e^{-\mathscr{H}} \mathrm{d} \lambda \\
& \leq-D\left(\sqrt{f_{t}}\right)
\end{aligned}
$$


assuming that the boundary term

$$
\int_{\partial \Xi} \partial_{i} h \partial_{i j}^{2} h e^{-\mathscr{H}}=0
$$

in the integration by parts vanishes.

To see (5.9), consider a segment $\lambda_{i}=\lambda_{i+1}$ of the boundary $\partial \Xi$. From the explicit representation (5.11), (5.12) in the next section, we will see that $f_{t} \geq 0$ is a meromorphic function in each variable in the domain $\Xi$ for any $t>0$. It can be represented as by $\left(\lambda_{i+1}-\lambda_{i}\right)^{\beta} F(\lambda)$ with some $\beta \in \mathbb{Z}$, where $F$ is analytic and $0<F<\infty$ near $\lambda_{i}=\lambda_{i+1}$. Since $f_{t} \geq 0$, we obtain that the exponent $\beta$ is non-negative and even. Therefore $f_{t}^{1 / 2}$ behaves as $\left|\lambda_{i+1}-\lambda_{i}\right|^{\beta / 2}$ with a non-negative integer exponent $\beta / 2$ near $\lambda_{i}=\lambda_{i+1}$. It then follows that $\partial_{i} \sqrt{f} \partial_{i j}^{2} \sqrt{f} e^{-\mathscr{H}}$ vanishes at the boundary due to the factor $\left(\lambda_{i+1}-\lambda_{i}\right)^{2}$ in $e^{-H}$, i.e. the integral (5.9) indeed vanishes.

\subsection{Bound on the entropy}

Lemma 5.1. Let $s=N^{-1}$. For any $\alpha>\frac{1}{4}$ we have

$$
S_{\mu}\left(f_{s}\right):=S\left(f_{s} \mu \mid \mu\right) \leq C N^{1+\alpha}
$$

with $C$ depending on $\alpha$.

Proof. In the proof we consider the probability density $u(\lambda)$ and the equilibrium measure $\mu$ extended to $\mathbb{R}^{N}$ (see (3.12) $)$, i.e. the eigenvalues are not ordered. Clearly $S\left(f_{s} \mu \mid \mu\right)=S\left(f_{s} \tilde{\mu} \mid \tilde{\mu}\right)$ and we estimate the relative entropy of the extended measures.

Given the density $f_{0}(\lambda) \tilde{\mu}(\mathrm{d} \lambda)$ of the eigenvalues of the Wigner matrix as an initial distribution, the eigenvalue density $f_{s}(\boldsymbol{\lambda})$ for the matrix evolved under the Dyson's Brownian motion is given by

$$
f_{s}(\boldsymbol{\lambda}) \widetilde{u}(\boldsymbol{\lambda})=\int_{\mathbb{R}^{N}} g_{s}(\boldsymbol{\lambda}, \boldsymbol{v}) f_{0}(\boldsymbol{v}) \widetilde{u}(\boldsymbol{v}) \mathrm{d} \boldsymbol{v}
$$

with a kernel

$$
g_{s}(\boldsymbol{\lambda}, \boldsymbol{v})=\frac{N^{N / 2}}{(2 \pi)^{N / 2} c^{N(N-1) / 2}\left(1-c^{2}\right)^{N / 2}} \frac{\Delta_{N}(\boldsymbol{\lambda})}{\Delta_{N}(\boldsymbol{v})} \operatorname{det}\left(\exp \left[\frac{-N\left(c \lambda_{j}-v_{k}\right)^{2}}{2\left(1-c^{2}\right)}\right]\right)_{j, k},
$$

where $c=c(s)=e^{-s / 2}$ for brevity. The derivation of (5.12) follows very similarly to Johansson's presentation of the Harish-Chandra/Itzykson-Zuber formula (see Proposition 1.1 of [21]) with the difference that in our case the matrix elements move by the Ornstein-Uhlenbeck process (3.1) instead of the Brownian motion.

In particular, formula (5.12) implies that $f_{s}$ is an analytic function for any $s>0$ since

$$
f_{s}(\boldsymbol{\lambda})=\frac{h_{s}(\boldsymbol{\lambda})}{\Delta_{N}(\boldsymbol{\lambda})} \int_{\mathbb{R}^{N}} \operatorname{det}\left(\exp \left[\frac{-N\left(c \lambda_{j}-v_{k}\right)^{2}}{2\left(1-c^{2}\right)}\right]\right)_{j, k} f_{0}(\boldsymbol{v}) \frac{\widetilde{u}(\boldsymbol{v})}{\Delta_{N}(\boldsymbol{v})} \mathrm{d} \boldsymbol{v}
$$

with an explicit analytic function $h_{s}(\boldsymbol{\lambda})$. Since the determinant is analytic in $\boldsymbol{\lambda}$, we see that $f_{s}(\boldsymbol{\lambda})$ is meromorphic in each variables and the only possible poles of $f_{s}(\lambda)$ come from the factors $\left(\lambda_{i}-\lambda_{j}\right)^{-1}$ 
in $\Delta_{N}(\lambda)$ near the coalescence points. But $f_{s}(\lambda)$ is a non-negative function, so it cannot have a singularity of order -1 , thus these singular factors cancel out from a factor $\left(\lambda_{i}-\lambda_{j}\right)$ from the integral. Alternatively, using the Laplace expansion the determinant, one can explicitly see that each 2 by 2 subdeterminant from the $i$-th and $j$-th columns carry a factor $\pm\left(\lambda_{i}-\lambda_{j}\right)$.

Then, by Jensen inequality from (5.11) and from the fact that $f_{0}(\boldsymbol{v}) \widetilde{u}(\boldsymbol{v})$ is a probability density, we have

$$
S_{\widetilde{\mu}}\left(f_{s}\right)=\int_{\mathbb{R}^{N}} f_{s}\left(\log f_{s}\right) \mathrm{d} \widetilde{\mu} \leq \iint_{\mathbb{R}^{N} \times \mathbb{R}^{N}} \log \left(\frac{g_{s}(\boldsymbol{\lambda}, \boldsymbol{v})}{\widetilde{u}(\boldsymbol{\lambda})}\right) g_{s}(\boldsymbol{\lambda}, \boldsymbol{v}) f_{0}(\boldsymbol{v}) \widetilde{u}(\boldsymbol{v}) \mathrm{d} \boldsymbol{\lambda} \mathrm{d} \boldsymbol{v} .
$$

Expanding this last expression we find, after an exact cancellation of the term $(N / 2) \log (2 \pi)$,

$$
\begin{aligned}
S_{\widetilde{\mu}}\left(f_{s}\right) \leq & \iint_{\mathbb{R}^{N} \times \mathbb{R}^{N}}\left\{\frac{N}{2} \log N-\frac{N(N-1)}{2} \log c-\frac{N}{2} \log \left(1-c^{2}\right)+\log \Delta_{N}(\boldsymbol{\lambda})-\log \Delta_{N}(\boldsymbol{v})\right. \\
& +\log \operatorname{det}\left(\exp \left[\frac{-N\left(c \lambda_{j}-v_{k}\right)^{2}}{2\left(1-c^{2}\right)}\right]\right)_{j, k}-\frac{N^{2}}{2} \log N \\
& +\frac{N}{2} \sum_{i=1}^{N} \lambda_{i}^{2}-2 \log \Delta_{N}(\boldsymbol{\lambda})+\sum_{j=1}^{N} \log j ! g_{s}(\boldsymbol{\lambda}, \boldsymbol{v}) f_{0}(\boldsymbol{v}) \widetilde{u}(\boldsymbol{v}) \mathrm{d} \boldsymbol{\lambda} \mathrm{d} \boldsymbol{v} .
\end{aligned}
$$

Since $s=N^{-1}$, we have $\log c=-1 / 2 N$ and $\log \left(1-c^{2}\right)=-\log N+O\left(N^{-1}\right)$. Hence

$$
\begin{aligned}
S_{\widetilde{\mu}}\left(f_{s}\right) \leq & \iint_{\mathbb{R}^{N} \times \mathbb{R}^{N}}\left\{C N \log N+\log \Delta_{N}(\boldsymbol{\lambda})-\log \Delta_{N}(\boldsymbol{v})+\log \operatorname{det}\left(\exp \left[\frac{-N\left(c \lambda_{j}-v_{k}\right)^{2}}{2\left(1-c^{2}\right)}\right]\right)_{j, k}\right. \\
& \left.-\frac{N^{2}}{2} \log N+\frac{N}{2} \sum_{i=1}^{N} \lambda_{i}^{2}-2 \log \Delta_{N}(\boldsymbol{\lambda})+\sum_{i=1}^{N} \log j !\right\} g_{s}(\boldsymbol{\lambda}, \boldsymbol{v}) f_{0}(\boldsymbol{v}) \widetilde{u}(\boldsymbol{v}) \mathrm{d} \boldsymbol{\lambda} \mathrm{d} \boldsymbol{v} .
\end{aligned}
$$

For the determinant term, we use that each entry is at most one, thus

$$
\log \operatorname{det}\left(\exp \left[\frac{-N\left(c \lambda_{j}-v_{k}\right)^{2}}{2\left(1-c^{2}\right)}\right]\right)_{j, k} \leq \log N !
$$

The last term in (5.13) can be estimated using Stirling's formula and Riemann integration

$$
\begin{aligned}
\sum_{j=1}^{N} \log j ! & \leq \sum_{j=1}^{N}\left(\log \left(\frac{j}{e}\right)^{j}+C \log (2 \pi j)\right) \\
& \leq \int_{1}^{N+1} \mathrm{~d} x x \log x-\sum_{j=1}^{N} j+C N \log N \\
& \leq \frac{N^{2} \log N}{2}-\frac{3}{4} N^{2}+C N \log N
\end{aligned}
$$

thus the $\frac{1}{2} N^{2} \log N$ terms cancel. For the $N^{2}$ terms we need the following approximation 
Lemma 5.2. With respect to any Wigner ensemble whose single-site distribution satisfies (2.4)-(2.6) and for any $\alpha>1 / 4$ we have

$$
\mathbb{E}\left[\frac{N}{2} \sum_{i=1}^{N} \lambda_{i}^{2}-2 \log \Delta_{N}(\lambda)\right]=\frac{3}{4} N^{2}+O\left(N^{1+\alpha}\right),
$$

where the constant in the error term depends on $\alpha$ and on the constants in (2.4)-(2.6).

Note that (2.6), (2.5) hold for both the initial Wigner ensemble with density $f_{0}$ and for the evolved one with density $f_{t}$. These conditions ensure that Theorem 3.5 of [15] is applicable.

Proof of Lemma 5.2. The quadratic term can be computed explicitly using (3.4):

$$
\frac{N}{2} \mathbb{E} \sum_{i=1}^{N} \lambda_{i}^{2}=\frac{N}{2} \mathbb{E} \operatorname{Tr} H^{2}=\frac{1}{2} N^{2}=\frac{N^{2}}{2} \int x^{2} \varrho_{s c}(x) \mathrm{d} x,
$$

The second (determinant) term will be approximated in the following lemma whose proof is postponed to Appendix $D$.

Lemma 5.3. With respect to any Wigner ensemble whose single-site distribution satisfies (2.4)-(2.6) and for any $\alpha>1 / 4$ we have

$$
\mathbb{E} \log \Delta_{N}(\boldsymbol{\lambda})=\frac{N^{2}}{2} \iint \log |x-y| \varrho_{s c}(x) \varrho_{s c}(y) \mathrm{d} x \mathrm{~d} y+O\left(N^{1+\alpha}\right) .
$$

Finally, explicit calculation then shows that

$$
\frac{1}{2} \int x^{2} \varrho_{s c}(x) \mathrm{d} x-\iint \log |x-y| \varrho_{s c}(x) \varrho_{s c}(y) \mathrm{d} x \mathrm{~d} y=\frac{3}{4},
$$

and this proves Lemma 5.2 .

Hence, continuing the estimate (5.13), we have the bound

$$
\begin{aligned}
S_{\widetilde{\mu}}\left(f_{s}\right) & \leq C N^{1+\alpha}+\iint_{\mathbb{R}^{N} \times \mathbb{R}^{N}}\left\{\log \Delta_{N}(\boldsymbol{\lambda})-\log \Delta_{N}(\boldsymbol{v})\right\} g_{s}(\boldsymbol{\lambda}, \boldsymbol{v}) f_{0}(\boldsymbol{v}) \widetilde{u}(\boldsymbol{v}) \mathrm{d} \boldsymbol{v} \mathrm{d} \boldsymbol{\lambda} \\
& \leq C N^{1+\alpha}+\frac{N}{4} \mathbb{E} \sum_{j=1}^{N}\left[\lambda_{j}^{2}(s)-\lambda_{j}^{2}(0)\right]=C N^{1+\alpha},
\end{aligned}
$$

where we used Lemma 5.2 both for the initial Wigner measure and for the evolved one and finally we used that the $\mathbb{E} \operatorname{Tr} H^{2}$ is preserved, see (3.4). This completes the proof of (5.10).

\section{Local equilibrium}

\subsection{External and internal points}

Choose $t=\tau N^{-1}$ with some $\tau \geq 2$. Thus from (5.4) with $s=N^{-1}$, we have

$$
D\left(\sqrt{f}_{t}\right) \leq 2 S\left(f_{N^{-1}}\right) t^{-1} \leq C N^{2+\alpha} \tau^{-1}
$$


by using (5.10). Recall that the eigenvalues are ordered, $\lambda_{1}<\lambda_{2}<\ldots<\lambda_{N}$. Let $L \leq N-n$ ( $n$ was defined in (4.15)) and define

$$
\Pi_{L}(\lambda):=\left\{\lambda_{L+1}, \lambda_{L+2}, \ldots \lambda_{L+n}\right\}
$$

and

$$
\Pi_{L}^{c}(\lambda):=\left\{\lambda_{1}, \lambda_{2}, \ldots \lambda_{N}\right\} \backslash \Pi_{L}(\lambda)
$$

its complement. For convenience, we will relabel the elements of $\Pi_{L}$ as $\mathbf{x}=\left\{x_{1}, x_{2}, \ldots x_{n}\right\}$ in increasing order. The elements of $\Pi_{L}^{c}$ will be denoted by

$$
\Pi_{L}^{c}(\boldsymbol{\lambda}):=\mathbf{y}=\left(y_{-L}, y_{-L+1}, \ldots y_{-1}, y_{1}, y_{2}, \ldots y_{N-L-n}\right) \in \Xi^{(N-n)},
$$

again in increasing order $(\Xi$ was defined in (3.11) $)$. We set

$$
J_{L}:=\{-L,-L+1, \ldots,-1,1,2, \ldots N-L-n\}
$$

to be the index set of the $y$ 's. We will refer to the $y$ 's as external points and to the $x_{j}$ 's as internal points. Note that the indices are chosen such that for any $j$ we have $y_{k}<x_{j}$ for $k<0$ and $y_{k}>x_{j}$ for $k>0$. In particular, for any fixed $L$, we can split any $\mathbf{y} \in \Xi^{(N-n)}$ as $\mathbf{y}=\left(\mathbf{y}_{-}, \mathbf{y}_{+}\right)$where

$$
\mathbf{y}_{-}:=\left(y_{-L}, y_{-L+1}, \ldots y_{-1}\right), \quad \mathbf{y}_{+}:=\left(y_{1}, y_{2}, \ldots y_{N-L-n}\right)
$$

The set $\Xi^{(N-n)}$ with a splitting mark after the $L$-th coordinate will be denoted by $\Xi_{L}^{(N-n)}$ and we use the $\mathbf{y} \in \Xi^{(N-n)} \Longleftrightarrow\left(\mathbf{y}_{-}, \mathbf{y}_{+}\right) \in \Xi_{L}^{(N-n)}$ one-to-one correspondance.

For a fixed $L$ we will often consider the expectation of functions $O(\mathbf{y})$ on $\Xi^{(N-n)}$ with respect to $\mu$ or $f \mu$; this will always mean the marginal probability:

$$
\begin{gathered}
\mathbb{E}_{\mu} O:=\int O(\mathbf{y}) u\left(\mathbf{y}_{-}, x_{1}, x_{2}, \ldots x_{n}, \mathbf{y}_{+}\right) \mathrm{d} \mathbf{y d} \mathbf{x}, \quad \mathbf{y}=\left(\mathbf{y}_{-}, \mathbf{y}_{+}\right) . \\
\mathbb{E}_{f} O:=\int O(\mathbf{y})(f u)\left(\mathbf{y}_{-}, x_{1}, x_{2}, \ldots x_{n}, \mathbf{y}_{+}\right) \mathrm{d} \mathbf{y d} \mathbf{x} .
\end{gathered}
$$

For a fixed $L \leq N-n$ and $\mathbf{y} \in \Xi^{(N-n)}$ let

$$
f_{\mathbf{y}}^{L}(\mathbf{x})=f_{\mathbf{y}}(\mathbf{x})=f_{t}(\mathbf{y}, \mathbf{x})\left[\int f_{t}(\mathbf{y}, \mathbf{x}) \mu_{\mathbf{y}}(\mathrm{d} \mathbf{x})\right]^{-1}
$$

be the conditional density of $\mathbf{x}$ given $\mathbf{y}$ with respect to the conditional equilibrium measure

$$
\mu_{\mathbf{y}}^{L}(\mathrm{~d} \mathbf{x})=\mu_{\mathbf{y}}(\mathrm{d} \mathbf{x})=u_{\mathbf{y}}(\mathbf{x}) \mathrm{d} \mathbf{x}, \quad u_{\mathbf{y}}(\mathbf{x}):=u(\mathbf{y}, \mathbf{x})\left[\int u(\mathbf{y}, \mathbf{x}) \mathrm{d} \mathbf{x}\right]^{-1}
$$

Here $f_{\mathbf{y}}^{L}$ also depends on time $t$, but we will omit this dependence in the notation. Note that for any fixed $\mathbf{y} \in \Xi^{(N-n)}$, any value $x_{j}$ lies in the interval $I_{\mathbf{y}}:=\left[y_{-1}, y_{1}\right]$, i.e. the functions $u_{\mathbf{y}}(\mathbf{x})$ and $f_{\mathbf{y}}(\mathbf{x})$ are supported on the set

$$
\Xi_{\mathbf{y}}^{(n)}:=\left\{\mathbf{x}=\left(x_{1}, x_{2}, \ldots, x_{n}\right): y_{-1}<x_{1}<x_{2}<\ldots<x_{n}<y_{1}\right\} \subset I_{\mathbf{y}}^{n} .
$$


Now we localize the good set $\Omega$ introduced in Definition 4.1. For any fixed $L$ and $\mathbf{y}=\left(\mathbf{y}_{-}, \mathbf{y}_{+}\right) \in$ $\Xi_{L}^{(N-n)}$ we define

$$
\Omega_{\mathbf{y}}:=\left\{\Pi_{L}(\lambda): \lambda \in \Omega, \Pi_{L}^{c}(\lambda)=\mathbf{y}\right\}=\left\{\mathbf{x}=\left(x_{1}, x_{2}, \ldots, x_{n}\right):\left(\mathbf{y}_{-}, \mathbf{x}, \mathbf{y}_{+}\right) \in \Omega\right\} .
$$

Set

$$
\Omega_{1}=\Omega_{1}(L):=\left\{\mathbf{y} \in \Xi_{L}^{(N-n)}: \mathbb{P}_{f_{\mathbf{y}}}\left(\Omega_{\mathbf{y}}\right) \geq 1-C e^{-n^{\gamma / 12}}\right\}
$$

Since

from (4.19) we have

$$
\mathbb{P}(\Omega)=\mathbb{P}_{f} \mathbb{P}_{f_{\mathrm{y}}}\left(\Omega_{\mathrm{y}}\right)
$$

$$
\mathbb{P}_{f}\left(\Omega_{1}\right) \geq 1-C e^{-n^{\gamma / 12}}
$$

Here $\mathbb{P}_{f}\left(\Omega_{1}\right)$ is a short-hand notation for the marginal expectation, i.e.

$$
\mathbb{P}_{f}\left(\Omega_{1}\right):=\mathbb{P}_{f}\left[\left(\Pi_{L}^{c}\right)^{-1}\left(\Omega_{1}\right)\right],
$$

but we will neglect this distinction.

Note that $\mathbf{y} \in \Omega_{1}$ also implies, for large $N$, that there exists an $\mathbf{x} \in I_{\mathbf{y}}^{n}$ such that $\left(\mathbf{y}_{-}, \mathbf{x}, \mathbf{y}_{+}\right) \in \Omega$. This ensures that those properties of $\lambda \in \Omega$ that are determined only by y's, will be inherited to the y's. E.g. $y \in \Omega_{1}$ will guarantee that the local density of y's is close to the semicircle law on each interval away from $I_{\mathrm{y}}$. More precisely, note that for any interval $I=\left[E-\eta_{m}^{*} / 2, E+\eta_{m}^{*} / 2\right]$ of length $\eta_{m}^{*}=2^{m} n^{\gamma} N^{-1}$ and center $E,|E| \leq 2-\kappa$, that is disjoint from $I_{\mathrm{y}}$, we have, by $(4.16)$,

$$
\mathbf{y} \in \Omega_{1}, \quad I \cap I_{\mathbf{y}}=\emptyset \quad \Longrightarrow \quad\left|\frac{\mathscr{N}(I)}{N|I|}-\varrho_{s c}(E)\right| \leq \frac{1}{(N|I|)^{1 / 4}} n^{\gamma / 12} .
$$

Moreover, for any interval $I$ with $|I| \geq n^{\gamma} N^{-1}$ we have, by (4.18),

$$
\mathbf{y} \in \Omega_{1}, \quad I \cap I_{\mathbf{y}}=\emptyset \quad \Longrightarrow \quad \mathscr{N}(I) \leq K N|I| .
$$

For any $L$ with $N \kappa^{3 / 2} \leq L \leq N\left(1-\kappa^{3 / 2}\right)$, let $E_{L}=\mathfrak{N}_{s c}^{-1}\left(L N^{-1}\right)$, i.e.

$$
N \int_{-2}^{E_{L}} \varrho_{s c}(\lambda) \mathrm{d} \lambda=L .
$$

Then we have

$$
-2+C \kappa \leq E_{L} \leq 2-C \kappa, \quad \varrho_{s c}\left(E_{L}\right) \geq c \kappa^{1 / 2}
$$

Using (4.21) and (4.22) from Lemma 4.3 on the set $\Omega$ (see (4.18)), we for any $\mathbf{y} \in \Omega_{1}(L)$ we have

$$
\left|y_{-1}-\mathfrak{N}_{s c}^{-1}\left(L N^{-1}\right)\right| \leq C n^{-\gamma / 6}, \quad|| I_{\mathbf{y}}\left|-\frac{n}{N \varrho_{s c}\left(E_{L}\right)}\right| \leq C N^{-1} n^{\gamma+3 / 4}
$$

in particular

$$
\left|y_{-1}\right|,\left|y_{1}\right| \leq 2-\kappa / 2 \text { and }\left|I_{\mathbf{y}}\right| \leq \frac{C n}{N}
$$

with $C=C(\kappa)$.

Let

$$
\Omega_{2}=\Omega_{2}(L)=\left\{\left(\mathbf{y}_{-}, \mathbf{y}_{+}\right) \in \Xi_{L}^{(N-n)},:\left|I_{\mathbf{y}}\right| \leq K n N^{-1}\right\}
$$

with some large constant $K$. On the set $\Omega$ we have $\left|I_{\mathrm{y}}\right| \leq K n / N$ (see (6.14)), thus $\Pi_{L}^{c}(\Omega) \subset \Omega_{2}(L)$, i.e.

$$
\mathbb{P}_{f}\left(\Omega_{2}\right) \geq 1-C e^{-n^{\gamma / 6}}
$$




\subsection{Localization of the Dirichlet form}

For any $L \leq N-n$ and any $\mathbf{y} \in \Xi_{L}^{(N-n)}$, we define the Dirichlet form

$$
D_{L, \mathbf{y}}(f):=\int \frac{1}{2 N}\left(\nabla_{\mathbf{x}} f\right)^{2} \mathrm{~d} \mu_{\mathbf{y}}^{L}(\mathbf{x})
$$

for functions $f=f(\mathbf{x})$ defined on $\Xi_{\mathbf{y}}^{(n)}$. Hence from (6.1) we have the inequality

$$
\frac{1}{N\left(1-2 \kappa^{3 / 2}\right)} \sum_{L=N \kappa^{3 / 2}}^{N\left(1-\kappa^{3 / 2}\right)} \mathbb{E}_{f_{t}} D_{L, \mathbf{y}}\left(\sqrt{f_{\mathbf{y}}(\mathbf{x})}\right) \leq C n N^{-1} D(\sqrt{f} t) \leq C N^{1+\alpha} n \tau^{-1}
$$

where the expectation $\mathbb{E}_{f_{t}}$ is defined similarly to (6.4), with $f$ replaced by $f_{t}$. In the first inequality in (6.17), we used the fact that, by (6.5) and (6.6),

$$
\begin{aligned}
\mathbb{E}_{f_{t}} D_{L, \mathbf{y}} & \left(\sqrt{f_{\mathbf{y}}(\mathbf{x})}\right) \\
& =\int \mathrm{d} \mathbf{x d} \mathbf{y} f_{t}(\mathbf{y}, \mathbf{x}) u(\mathbf{y}, \mathbf{x}) D_{L, \mathbf{y}}\left(\sqrt{f_{\mathbf{y}}(\mathbf{x})}\right) \\
& =\frac{1}{8 N} \int \mathrm{d} \mathbf{x} \mathrm{d} \mathbf{y} f_{t}(\mathbf{y}, \mathbf{x}) u(\mathbf{y}, \mathbf{x})\left[\int \mathrm{d} \mathbf{x}^{\prime} \frac{\left|\nabla_{x^{\prime}} f_{t}\left(\mathbf{y}, \mathbf{x}^{\prime}\right)\right|^{2}}{f_{t}\left(\mathbf{y}, \mathbf{x}^{\prime}\right)} \frac{1}{\int \mathrm{d} \mathbf{x}^{\prime} f_{t}\left(\mathbf{y}, \mathbf{x}^{\prime}\right) u\left(\mathbf{y}, \mathbf{x}^{\prime}\right)} u\left(\mathbf{y}, \mathbf{x}^{\prime}\right)\right] \\
& =\frac{1}{8 N} \sum_{j=1}^{n} \int \mathrm{d} \mathbf{x} \mathrm{d} \mathbf{y} \frac{\left|\nabla_{x_{j}} f_{t}(\mathbf{y}, \mathbf{x})\right|^{2}}{f_{t}(\mathbf{y}, \mathbf{x})} u(\mathbf{y}, \mathbf{x})
\end{aligned}
$$

and therefore, when we sum over all $L \in\left\{N \kappa^{3 / 2}, \ldots, N\left(1-\kappa^{3 / 2}\right)\right\}$ as on the 1.h.s. of (6.17), every local Dirichlet form is summed over at most $n$ times, so we get the total Dirichlet form with a multiplicity at most $n$.

We define the set

$$
\mathscr{G}_{1}=\left\{N \kappa^{3 / 2} \leq L \leq N\left(1-\kappa^{3 / 2}\right): \mathbb{E}_{f_{t}} D_{L, \mathbf{y}}\left(\sqrt{f_{\mathbf{y}}(\mathbf{x})}\right) \leq C N^{1+\alpha} n^{2} \tau^{-1}\right\},
$$

then the above inequality guarantees that for the cardinality of $\mathscr{G}_{1}$,

$$
\frac{\left|\mathscr{G}_{1}\right|}{N\left(1-2 \kappa^{3 / 2}\right)} \geq 1-\frac{C}{n} \text {. }
$$

For $L \in \mathscr{G}_{1}$, we define

$$
\Omega_{3}=\Omega_{3}(L):=\left\{\left(\mathbf{y}_{-}, \mathbf{y}_{+}\right) \in \Xi_{L}^{(N-n)}: D_{L, \mathbf{y}}\left(\sqrt{f_{\mathbf{y}}(\mathbf{x})}\right) \leq C N^{1+\alpha} n^{4} \tau^{-1}\right\}
$$

then

$$
\mathbb{P}_{f}\left(\Omega_{3}^{c}\right) \leq C n^{-2}
$$




\subsection{Local entropy bound}

Suppose that $L \in \mathscr{G}_{1}$ and fix it. For any $\mathbf{y} \in \Xi_{L}^{(N-n)}$ denote by

$$
\mathscr{H}_{\mathbf{y}}(\mathbf{x})=N\left[\sum_{i=1}^{n} \frac{1}{2} x_{i}^{2}-\frac{2}{N} \sum_{1 \leq i<j \leq n} \log \left|x_{j}-x_{i}\right|-\frac{2}{N} \sum_{k \in J_{L}} \sum_{i=1}^{n} \log \left|x_{i}-y_{k}\right|\right]
$$

Note that

$$
\text { Hess } \mathscr{H}_{\mathbf{y}}(\mathbf{x}) \geq \inf _{x \in I_{\mathbf{y}}} \sum_{k \in J_{L}}\left|x-y_{k}\right|^{-2}
$$

for any $\mathbf{x} \in I_{\mathbf{y}}^{n}$ as a matrix inequality. On the set $\mathbf{y} \in \Omega_{2}(L)$ we have

$$
\inf _{x \in I_{\mathrm{y}}} \sum_{k \in J_{L}}\left|x-y_{k}\right|^{-2} \geq \frac{1}{\left|y_{1}-y_{-1}\right|^{2}} \geq \frac{c N^{2}}{n^{2}}, \quad \mathbf{y} \in \Omega_{2}(L) .
$$

We can apply the logarithmic Sobolev inequality (5.3) to the local measure $\mu_{\mathrm{y}}$, taking into account Remark 5.1, Thus we have

$$
S_{\mu_{\mathbf{y}}^{L}}\left(f_{\mathbf{y}}\right) \leq c^{-1} n^{2} N^{-1} D_{L, \mathbf{y}}\left(\sqrt{f_{\mathbf{y}}(\mathbf{x})}\right) \leq C n^{6} N^{\alpha} \tau^{-1} \quad \text { for any } \mathbf{y} \in \Omega_{2}(L) \cap \Omega_{3}(L), L \in \mathscr{G}_{1} .
$$

Using the inequality

$$
\sqrt{S(f)} \geq C \int|f-1| \mathrm{d} \mu
$$

for $\mu=\mu_{\mathbf{y}}$ and $f=f_{\mathbf{y}}$, we have also have

$$
\left[\int\left|f_{\mathbf{y}}-1\right| \mathrm{d} \mu_{\mathbf{y}}\right]^{2} \leq C n^{6} N^{\alpha} \tau^{-1} \quad \text { for any } \mathbf{y} \in \Omega_{2}(L) \cap \Omega_{3}(L), L \in \mathscr{G}_{1}
$$

We will choose $t=N^{-1} \tau$ with $\tau=N^{\beta}$ such that

$$
C n^{6} N^{\alpha} \tau^{-1} \leq n^{-4}
$$

i.e. $\beta \geq 10 \varepsilon+\alpha$.

\subsection{Good external configurations}

Definition 6.1. The set of good $L$-indices is defined by

$$
\begin{aligned}
\mathscr{G}:= & \left\{L \in \mathscr{G}_{1}: \mathbb{E}_{f} \sum_{j \neq L} \frac{\mathbf{1}_{\Omega}}{\left[N\left(\lambda_{j}-\lambda_{L}\right)\right]^{2}} \leq C n^{3 \gamma}, \mathbb{E}_{f} \sum_{j \neq L+n+1} \frac{\mathbf{1}_{\Omega}}{\left[N\left(\lambda_{j}-\lambda_{L+n+1}\right)\right]^{2}} \leq C n^{3 \gamma}\right\} \\
& \cap\left\{L \in \mathscr{G}_{1}: \mathbb{E}_{f} \sum_{j \neq L} \frac{\mathbf{1}_{\Omega}}{N\left|\lambda_{j}-\lambda_{L}\right|} \leq C n^{3 \gamma}, \quad \mathbb{E}_{f} \sum_{j \neq L+n+1} \frac{\mathbf{1}_{\Omega}}{N\left|\lambda_{L+n+1}-\lambda_{j}\right|} \leq C n^{3 \gamma}\right\} .
\end{aligned}
$$


Lemma 4.4 together with (6.19) imply that

$$
\frac{|\mathscr{G}|}{N\left(1-2 \kappa^{3 / 2}\right)} \geq 1-\frac{1}{n^{\gamma}}
$$

Notice that for any fixed $L$ we can write

$$
\begin{gathered}
\mathbb{E}_{f} \sum_{j=L+1}^{L+n} \frac{\mathbf{1}_{\Omega}}{N\left(\lambda_{j}-\lambda_{L}\right)}=\mathbb{E}_{f} \mathbb{E}_{f_{\mathrm{y}}} \sum_{j=1}^{n} \frac{\mathbf{1}_{\Omega_{\mathrm{y}}}}{N\left(x_{j}-y_{-1}\right)} \\
\mathbb{E}_{f} \sum_{j=L+1}^{L+n} \frac{\mathbf{1}_{\Omega}}{\left[N\left(\lambda_{j}-\lambda_{L}\right)\right]^{2}}=\mathbb{E}_{f} \mathbb{E}_{f_{\mathrm{y}}} \sum_{j=1}^{n} \frac{\mathbf{1}_{\Omega_{\mathrm{y}}}}{\left[N\left(x_{j}-y_{-1}\right)\right]^{2}}
\end{gathered}
$$

and we also have

$$
\mathbb{E}_{f} \sum_{j \neq L+1, \ldots L+n} \frac{\mathbf{1}_{\Omega}}{N\left|\lambda_{j}-\lambda_{L}\right|}=\mathbb{E}_{f} \sum_{j \in J_{L}, j \neq-1} \frac{1}{N\left|y_{j}-y_{-1}\right|} \mathbb{P}_{f_{\mathrm{y}}}\left(\Omega_{\mathrm{y}}\right) \geq \frac{1}{2} \mathbb{E}_{f} \sum_{j \in J_{L}, j \neq-1} \frac{\mathbf{1}\left(\mathbf{y} \in \Omega_{1}\right)}{N\left|y_{j}-y_{-1}\right|},
$$

and similar formulae hold when $\lambda_{L}$ is replaced with $\lambda_{L+n+1}$ and $y_{-1}$ with $y_{1}$.

We also want to ensure that the density on scale $\eta:=\eta_{0}^{*}=n^{\gamma} N^{-1}$ is close to the semicircle law. Let

$$
\mathscr{O}_{E}(x)=\mathbf{1}(|x-E| \leq \eta / 2)
$$

be the characteristic function of the interval $[E-\eta / 2, E+\eta / 2]$. Consider $\Omega^{(0)}$ defined in (4.16), then $\Omega \subset \Omega^{(0)}$ and (4.19) imply that

$$
\mathbb{E}_{f} \mathbf{1}_{\Omega} \sup _{|E| \leq 2-\kappa / 2}\left|\frac{1}{N \eta} \sum_{i=1}^{N} \mathscr{O}_{E}\left(\lambda_{i}\right)-\varrho_{s c}(E)\right| \leq(N \eta)^{-1 / 4} n^{\gamma / 12}=n^{-\gamma / 6}
$$

Fix $L \in \mathscr{G}$, consider $\mathbf{y} \in \Xi_{L}^{(N-n)}$ and define

$$
I_{\mathrm{y}}^{*}:=\left[y_{-1}+\eta / 2, y_{1}-\eta / 2\right]
$$

so that if $E \in I_{\mathbf{y}}^{*}$ then $[E-\eta / 2, E+\eta / 2] \subset I_{\mathrm{y}}$. Moreover, on the set $\Omega$ we know that $I_{\mathrm{y}} \subset[-2+$ $\kappa / 2,2-\kappa / 2]$ (see (6.14)). Therefore

$$
\mathbb{E}_{f} \mathbb{E}_{f_{\mathbf{y}}} \mathbf{1}_{\Omega_{\mathrm{y}}} \sup _{E \in I_{\mathbf{y}}^{*}}\left|n^{-\gamma} \sum_{i=1}^{n} \mathscr{O}_{E}\left(x_{i}\right)-\varrho_{s c}(E)\right| \leq \mathbb{E}_{f} \mathbf{1}_{\Omega} \sup _{|E| \leq 2-\kappa / 2}\left|\frac{1}{N \eta} \sum_{i=1}^{N} \mathscr{O}_{E}\left(\lambda_{i}\right)-\varrho_{s c}(E)\right| \leq n^{-\gamma / 6} .
$$

This gives rise to the following definition:

Definition 6.2. Let $L \in \mathscr{G}$. The set of good external points is given by

$$
\begin{aligned}
\mathscr{Y}_{L}:= & \Omega_{1} \cap \Omega_{2} \cap \Omega_{3} \cap\left\{\mathbf{y}=\left(\mathbf{y}_{-}, \mathbf{y}_{+}\right) \in \Xi_{L}^{(N-n)}: \sum_{ \pm} \sum_{\substack{k \in J_{L} \\
k \neq \pm 1}} \frac{1}{\left|N\left(y_{ \pm 1}-y_{k}\right)\right|} \leq C n^{3 \gamma},\right. \\
& \mathbb{E}_{f_{\mathbf{y}}} \sum_{ \pm} \sum_{j=1}^{n} \frac{\mathbf{1}_{\Omega_{\mathbf{y}}}}{N\left|x_{j}-y_{ \pm 1}\right|} \leq C n^{4 \gamma}, \mathbb{E}_{f_{\mathbf{y}}} \sum_{ \pm} \sum_{j=1}^{n} \frac{\mathbf{1}_{\Omega_{\mathrm{y}}}}{\left[N\left(x_{j}-y_{ \pm 1}\right)\right]^{2}} \leq C n^{4 \gamma}, \\
& \left.\mathbb{E}_{f_{\mathbf{y}}} \mathbf{1}_{\Omega_{\mathbf{y}}} \sup _{E \in I_{\mathbf{y}}^{*}}\left|n^{-\gamma} \sum_{i=1}^{n} \mathbf{1}\left(N\left|x_{i}-E\right| \leq \frac{1}{2} n^{\gamma}\right)-\varrho_{s c}(E)\right| \leq n^{-\gamma / 12}\right\}
\end{aligned}
$$


It follows from (6.8), 6.16), 6.21), 6.28) and (6.30) that

$$
\mathbb{P}_{f}\left(\mathscr{Y}_{L}\right) \geq 1-C n^{-\gamma / 12} \text {. }
$$

\subsection{Bounds in equilibrium}

In this section we translate the bounds in the second and third lines of (6.31) into similar bounds with respect to equilibrium using that the control on the local Dirichlet form also controls the local entropy for the good indices:

Lemma 6.1. Let $A>0$ be arbitrary and $\mathbf{y} \in \mathscr{Y}_{L}$. If $\tau \geq n^{4 A+8} N^{\alpha}$, i.e. $\beta \geq(4 A+8) \varepsilon+\alpha$, then for $p=1,2$ we have

$$
\mathbb{E}_{\mu_{\mathrm{y}}} \sum_{ \pm} \sum_{j=1}^{n} \frac{\mathbf{1}\left(N\left|x_{j}-y_{ \pm 1}\right| \geq n^{-A}\right)}{\left[N\left|x_{j}-y_{ \pm 1}\right|\right]^{p}} \leq C n^{4 \gamma}
$$

Moreover, we also have

$$
\mathbb{E}_{\mu_{\mathrm{y}}} \sup _{E \in I_{\mathrm{y}}^{*}}\left|n^{-\gamma} \sum_{i=1}^{n} \mathscr{O}_{E}\left(x_{i}\right)-\varrho_{s c}(E)\right| \leq C n^{-\gamma / 12}
$$

Proof. Let $\mathscr{O}: \mathbb{R}^{n} \rightarrow \mathbb{R}$ be any observable and $\Omega_{\mathrm{y}}$ be any event. Then for any fixed $\mathbf{y} \in \Xi^{(N-n)}$ we have

$$
\left|\mathbb{E}_{f_{\mathrm{y}}} \mathbf{1}_{\Omega_{\mathrm{y}}} \mathscr{O}-\mathbb{E}_{\mu_{\mathrm{y}}} \mathbf{1}_{\Omega_{\mathrm{y}}} \mathscr{O}\right|^{2}=\left[\int \mathbf{1}_{\Omega_{\mathrm{y}}} \mathscr{O}\left(f_{\mathrm{y}}-1\right) \mathrm{d} \mu_{\mathrm{y}}\right]^{2} \leq\|O\|_{\infty}^{2}\left[\int\left|f_{\mathrm{y}}-1\right| \mathrm{d} \mu_{\mathrm{y}}\right]^{2} \leq C\|O\|_{\infty}^{2} S_{\mu_{\mathrm{y}}}\left(f_{\mathrm{y}}\right)
$$

by the entropy inequality (6.25). If $L \in \mathscr{G}$ and $\mathbf{y} \in \Omega_{2}(L)$, then we have by (6.26) that

$$
\mathbb{E}_{\mu_{\mathrm{y}}} \mathbf{1}_{\Omega_{\mathrm{y}}} \mathscr{O} \leq \mathbb{E}_{f_{\mathrm{y}}} \mathbf{1}_{\Omega_{\mathrm{y}}} \mathscr{O}+C\|\mathscr{O}\|_{\infty}\left(n^{6} N^{\alpha} \tau^{-1}\right)^{1 / 2}
$$

For a given $\mathbf{y} \in \mathscr{Y}_{L}$, we set the observable

$$
\mathscr{O}(\mathbf{x})=\sum_{ \pm} \sum_{j=1}^{n} \frac{\mathbf{1}\left(N\left(x_{j}-y_{ \pm 1}\right) \geq n^{-A}\right)}{\left[N\left|x_{j}-y_{ \pm 1}\right|\right]^{p}}
$$

with $\|\mathscr{O}\|_{\infty} \leq C n^{A p+1} \leq c n^{2 A+1}$. Then, for $\tau \geq n^{4 A+8} N^{\alpha}$ we obtain from (6.31) and (6.35) that

$$
\mathbb{E}_{\mu_{\mathrm{y}}} \sum_{ \pm} \sum_{j=1}^{n} \frac{\mathbf{1}_{\Omega_{\mathrm{y}}} \mathbf{1}\left(N\left|x_{j}-y_{ \pm 1}\right| \geq n^{-A}\right)}{\left[N\left|x_{j}-y_{ \pm 1}\right|\right]^{p}} \leq C n^{4 \gamma}+C n^{2 A+4} N^{\alpha / 2} \tau^{-1 / 2} \leq C n^{4 \gamma} .
$$

On the complement set $\Omega_{\mathrm{y}}^{c}$ we just use the crude supremum bound together with the bound on $\mathbb{P}_{f_{\mathrm{y}}}\left(\Omega_{\mathrm{y}}^{c}\right)$ in the definition of $\Omega_{1}$ (6.7):

$$
\mathbb{E}_{\mu_{\mathrm{y}}} \sum_{ \pm} \sum_{j=1}^{n} \frac{\mathbf{1}_{\Omega_{\mathrm{y}}^{c}} \mathbf{1}\left(N\left|x_{j}-y_{ \pm 1}\right| \geq n^{-A}\right)}{\left[N\left|x_{j}-y_{ \pm 1}\right|\right]^{p}} \leq C n^{4 A+1} e^{-n^{\gamma / 12}} \leq C n^{4 \gamma}
$$

Combining the last two estimates proves 6.33).

The proof of (6.34) is analogous, here we use that the corresponding observable has an $L^{\infty}$ bound

$$
\left|n^{-\gamma} \sum_{i=1}^{n} \mathscr{O}_{E}\left(x_{i}\right)-\varrho_{s c}(E)\right| \leq n^{1-\gamma}
$$

This completes the proof of Lemma 6.1. 


\section{Cutoff Estimates}

In this section, we cutoff the interaction with the far away particles. We fix a good index $L \in \mathscr{G}$ and a good external point configuration $\mathbf{y} \in \mathscr{Y}_{L}$. Consider the measure $\mu_{\mathbf{y}}=e^{-\mathscr{H}_{\mathbf{y}}} / Z_{\mathbf{y}}$ with

$$
\mathscr{H}_{\mathbf{y}}(\mathbf{x})=N\left[\sum_{i=1}^{n} x_{i}^{2} / 2-2 N^{-1} \sum_{1 \leq i<j \leq n} \log \left|x_{j}-x_{i}\right|-2 N^{-1} \sum_{k, i} \log \left|x_{i}-y_{k}\right|\right]
$$

The measure $\mu_{\mathbf{y}}$ is supported on the interval $I_{\mathbf{y}}=\left(y_{-1}, y_{1}\right)$.

For any fixed $\mathbf{y}$, decompose

$$
\mathscr{H}_{\mathbf{y}}=\mathscr{H}_{1}+\mathscr{H}_{2}, \quad \mathscr{H}_{2}(\mathbf{x})=\sum_{i=1}^{n} V_{2}\left(x_{i}\right)
$$

where

$$
V_{2}(x)=\frac{N}{2} x^{2}-2 \sum_{|k| \geq n^{B}} \log \left|x-y_{k}\right|
$$

and

$$
\mathscr{H}_{1}(\mathbf{x})=-2 \sum_{1 \leq i<j \leq n} \log \left|x_{j}-x_{i}\right|-\sum_{i=1}^{n} V_{1}\left(x_{i}\right)
$$

with

$$
V_{1}(x)=-2 \sum_{|k|<n^{B}} \log \left|x-y_{k}\right|
$$

where $B$ is a large positive number with $B \varepsilon<1 / 2$. We define the measure

$$
\mu_{\mathbf{y}}^{(1)}(\mathrm{d} \mathbf{x}):=\frac{e^{-\mathscr{H}_{1}(\mathbf{x})} \mathrm{d} \mathbf{x}}{Z_{1}} .
$$

Lemma 7.1. Let $L \in \mathscr{G}$ and $\mathbf{y} \in \mathscr{Y}_{L}$. For $B \geq 20$, we have

$$
\sup _{\mathbf{x} \in I_{\mathbf{y}}^{n}}\left|\frac{\mathrm{d} \mu_{\mathbf{y}}^{(1)}}{\mathrm{d} \mu_{\mathbf{y}}}(\mathbf{x})-1\right| \leq C n^{-B / 9+2}
$$

This lemma will imply that one can cutoff all $y_{k}$ 's in the potential with $|k| \geq n^{B}$.

Proof. Let

$$
\delta V_{2}:=\max _{x \in I_{\mathrm{y}}} V_{2}-\min _{x \in I_{\mathrm{y}}} V_{2}
$$

then, by (6.15) and $\mathbf{y} \in \mathscr{Y}_{L}$, we have

$$
\delta V_{2} \leq\left|I_{\mathbf{y}}\right|\left\|V_{2}^{\prime}\right\|_{\infty} \leq C n N^{-1}\left\|V_{2}^{\prime}\right\|_{\infty}
$$

In Lemma 7.2 we will give an upper bound on $\left\|V_{2}^{\prime}\right\|_{\infty}$, and then we have, for $B \geq 20$, that

$$
\delta V_{2} \leq C n^{-B / 9+1}
$$


Since

$$
\left|\frac{\mathrm{d} \mu_{\mathbf{y}}^{(1)}}{\mathrm{d} \mu_{\mathbf{y}}}(\mathbf{x})-1\right|=\left|e^{-\sum_{i=1}^{n}\left[V_{2}\left(x_{i}\right)-\min V_{2}\right]}-1\right| \leq C n \delta V_{2} \leq C n^{-B / 9+2},
$$

we obtain (7.6).

Lemma 7.2. For $B \geq 20$ and for any $L \in \mathscr{G}_{1}, \mathbf{y} \in \mathscr{Y}_{L}$ we have

$$
\sup _{x \in I_{\mathrm{y}}}\left|V_{2}^{\prime}(x)\right|=\sup _{x \in I_{\mathrm{y}}}\left|-2 \sum_{|k| \geq n^{B}} \frac{1}{x-y_{k}}+N x\right| \leq C N n^{\gamma / 12-B / 8} .
$$

Proof. Recall that $\mathbf{y} \in \mathscr{Y}_{L} \subset \Omega_{1}$ implies that the density of the $y$ 's is close the semicircle law in the sense of (6.9). Let

$$
d:=\frac{n^{B}}{N \varrho_{s c}\left(y_{-1}\right)} .
$$

Since $\mathbf{y} \in \Omega_{1}$, we know that $\left|y_{-1}\right|,\left|y_{1}\right| \leq 2-\kappa / 2$ (see (6.14)), thus $\varrho_{s c}\left(y_{-1}\right) \geq c>0$. Taking the imaginary part of $(4.3)$ for $|z| \leq 2$ and renaming the variables, we have the identity

$$
x=2 \int_{\mathbb{R}} \frac{\varrho_{s c}(y)}{x-y} \mathrm{~d} y .
$$

Furthermore, with $\bar{y}=\frac{1}{2}\left(y_{-1}+y_{1}\right)$ we have

$$
\left|\int_{|y-\bar{y}| \leq d} \frac{\varrho_{s c}(y)}{x-y} \mathrm{~d} y\right| \leq C d
$$

since $\bar{y}$ is away from the spectral edge thus $\varrho_{s c}$ is continuously differentiable on the interval of integration $[\bar{y}-d, \bar{y}+d]$. Thus

$$
\left|N x-2 N \int_{|y-\bar{y}| \geq d} \frac{\varrho_{s c}(y)}{x-y} \mathrm{~d} y\right| \leq C N d \leq C n^{B}
$$

therefore to prove $(7.7)$ it is sufficient to show that

$$
\sup _{x \in I_{\mathrm{y}}}\left|\frac{1}{N} \sum_{|k| \geq n^{B}} \frac{1}{x-y_{k}}-\int_{|y-\bar{y}| \geq d} \frac{\varrho_{s c}(y)}{x-y} \mathrm{~d} y\right| \leq C n^{\gamma / 12-B / 8}
$$

We will consider only $k \geq n^{B}$ and compare the sum with the integral on the regime $y \geq \bar{y}+d$, the sum for $k \leq-n^{B}$ is similar.

Define dyadic intervals

$$
I_{m}=\left[\bar{y}+2^{m} d, \bar{y}+2^{m+1} d\right], \quad m=0,1,2, \ldots, \log N
$$

Since $\mathbf{y} \in \mathscr{Y}_{L} \subset \Omega_{1}$, i.e. $\max \left|y_{k}\right| \leq K$, there will be no $y_{k}$ above the last interval $I_{\log N}$. We subdivide each $I_{m}$ into $n^{B / 2}$ equal disjoint subintervals of length $2^{m} d n^{-B / 2}$

$$
I_{m}=\bigcup_{\ell=1}^{n^{B / 2}} I_{m, \ell}, \quad I_{m, \ell}=\left[y_{m, \ell-1}^{*}, y_{m, \ell}^{*}\right] \quad \text { with } \quad y_{m, \ell}^{*}:=y_{1}+2^{m} d\left(1+\ell n^{-B / 2}\right) .
$$


For $\mathbf{y} \in \mathscr{Y}_{L} \subset \Omega_{1}$, the estimate (4.22) holds for $y_{1}$ and $y_{n^{B}}$, i.e.

$$
\left|N \varrho_{s c}\left(y_{1}\right)\left(y_{n^{B}}-y_{1}\right)-\left(n^{B}-1\right)\right| \leq C n^{\gamma+3 B / 4} \leq C n^{4 B / 5}
$$

if $B \geq 20$, which means that

$$
\left|y_{n^{B}}-\left(y_{1}+d\right)\right| \leq \frac{C n^{4 B / 5}}{N}+\frac{n^{B}}{N}\left|\frac{1}{\varrho_{s c}\left(y_{-1}\right)}-\frac{1}{\varrho_{s c}\left(y_{1}\right)}\right| \leq \frac{C n^{4 B / 5}}{N}+\frac{C n^{B+1}}{N^{2}} \leq \frac{C n^{4 B / 5}}{N}
$$

(using $B \varepsilon<1 / 2, n^{B} \leq N^{1 / 2}$ ), i.e.

$$
\left|y_{n^{B}}-(\bar{y}+d)\right| \leq \frac{C n^{4 B / 5}}{N}
$$

by using the definition of $d$ from (7.8), the fact that $\varrho_{s c}\left(y_{ \pm 1}\right)$ is separated away from zero and that $\left|I_{\mathrm{y}}\right| \leq C n N^{-1}$ from (6.14).

Therefore we can estimate

$$
\begin{aligned}
\mid \frac{1}{N} \sum_{k \geq n^{B}} \frac{1}{x-y_{k}}- & \frac{1}{N} \sum_{m=0}^{\log N} \sum_{\ell=1}^{n^{B / 2}} \sum_{j \in J_{L}} \frac{\mathbf{1}\left(y_{j} \in I_{m, \ell}\right)}{x-y_{j}} \mid \\
& \leq \frac{1}{N} \sum_{j \in J_{L}} \frac{\mathbf{1}\left(j<n^{B}, y_{j} \geq \bar{y}+d\right)}{\left|x-y_{j}\right|}+\frac{1}{N} \sum_{j \in J_{L}} \frac{\mathbf{1}\left(j \geq n^{B}, y_{j}<\bar{y}+d\right)}{\left|x-y_{j}\right|} \\
& \leq C n^{1-B / 5} .
\end{aligned}
$$

To see the last estimate, we notice that in the first summand we have $\bar{y}+d \leq y_{j} \leq y_{n^{B}} \leq \bar{y}+d+$ $C n^{4 B / 5} N^{-1}$ by (7.11), i.e. all these $y_{j}$ 's lie in an interval of length $C n^{4 B / 5} N^{-1}$, so their number is bounded by $C n^{4 B / 5}$ by (6.10). Thus the first term in the right hand side of $(7.12)$ is bounded by $C n^{4 B / 5} N^{-1} d^{-1} \leq C n^{1-B / 5}$; the estimate of the second term is similar.

Using that

$$
\left|\max _{y \in I_{m, \ell}} \frac{1}{x-y}-\min _{y \in I_{m, \ell}} \frac{1}{x-y}\right| \leq\left|I_{m, \ell}\right| \max _{x \in I_{\mathrm{y}}} \max _{y \in I_{m, \ell}} \frac{1}{(x-y)^{2}} \leq C \frac{2^{m} d n^{-B / 2}}{\left(2^{m} d\right)^{2}} \leq \frac{C}{2^{m} d n^{B / 2}}
$$

we have

$$
\begin{aligned}
\mid \frac{1}{N} \sum_{m=0}^{\log N} \sum_{\ell=1}^{n^{B / 2}} \sum_{j \in J_{L}} \frac{\mathbf{1}\left(y_{j} \in I_{m, \ell}\right)}{x-y_{j}}-\frac{1}{N} \sum_{m=0}^{\log N} \sum_{\ell=1}^{n^{B / 2}} \frac{\mathcal{N}\left(I_{m, \ell}\right)}{x-y_{m, \ell}^{*}} & \leq \frac{C}{N} \sum_{m=0}^{\log N} \sum_{\ell=1}^{n^{B / 2}} \frac{\mathcal{N}\left(I_{m, \ell}\right)}{2^{m} d n^{B / 2}} \\
& \leq C \sum_{m=0}^{\log N} \sum_{\ell=1}^{n^{B / 2}} \frac{1}{n^{B}} \\
& \leq C n^{-B / 2} \log N \leq C n^{-B / 4}
\end{aligned}
$$

In the second line we used that $\mathscr{N}\left(I_{m, \ell}\right) \leq K N\left|I_{m, \ell}\right|$ by 6.10$)$ since $\mathbf{y} \in \Omega_{1}$ and $I_{m, \ell} \cap I_{\mathbf{y}}=\emptyset$. 
We use that for $\mathbf{y} \in \Omega_{1}$ we can apply (6.9) for $I=I_{m, \ell}$ and we get

$$
\begin{aligned}
\left|\frac{1}{N} \sum_{m \geq 0} \sum_{\ell=1}^{n^{B / 2}} \frac{\mathscr{N}\left(I_{m, \ell}\right)}{x-y_{m, \ell}^{*}}-\frac{1}{N} \sum_{m=0}^{\log N} \sum_{\ell=1}^{n^{B / 2}} \frac{N\left|I_{m, \ell}\right| \varrho_{s c}\left(y_{m, \ell}^{*}\right)}{x-y_{m, \ell}^{*}}\right| & \leq \frac{C n^{\gamma / 12}}{N} \sum_{m=0}^{\log N} \sum_{\ell=1}^{n^{B / 2}} \frac{\left(N\left|I_{m, \ell}\right|\right)^{3 / 4}}{\left|x-y_{m, \ell}^{*}\right|} \\
& \leq \frac{C n^{\gamma / 12}}{N} \sum_{m=0}^{\log N} \sum_{\ell=1}^{n^{B / 2}} \frac{\left(2^{m} n^{B / 2}\right)^{3 / 4}}{2^{m} n^{B} N^{-1}} \\
& \leq C n^{\gamma / 12-B / 8},
\end{aligned}
$$

where we used that $\left|I_{m, \ell}\right|=2^{m} d n^{-B / 2} \leq C \cdot 2^{m} n^{B / 2} N^{-1}$ (see (7.8)) and that $\left|x-y_{m, \ell}^{*}\right| \geq 2^{m-1} d \geq$ $c \cdot 2^{m} n^{B} N^{-1}$.

Finally, the second term on the left hand side of (7.14) is a Riemann sum of the integral in (7.9) with an error

$$
\left|\sum_{m=0}^{\log N} \sum_{\ell=1}^{n^{B / 2}} \frac{\left|I_{m, \ell}\right| \varrho_{s c}\left(y_{m, \ell}^{*}\right)}{x-y_{m, \ell}^{*}}-\int_{|y-\bar{y}| \geq d} \frac{\varrho_{s c}(y)}{x-y} \mathrm{~d} y\right| \leq \sum_{m=0}^{\log N} \sum_{\ell=1}^{n^{B / 2}} C\left(\frac{N}{2^{m} n^{B}}\right)^{2}\left|I_{m, \ell}\right|^{2} \leq C n^{-B / 2} \log N,
$$

since on each interval $I_{m, \ell}$ we could estimate the derivative of the integrand as

$$
\sup _{y \in I_{m, \ell}}\left|\frac{\mathrm{d}}{\mathrm{d} y} \frac{\varrho_{s c}(y)}{x-y}\right| \leq C\left(\frac{N}{2^{m} n^{B}}\right)^{2}
$$

Combining (7.12), (7.13), (7.14) and (7.15), we have proved (7.9) which completes the proof of Lemma 7.2 .

\section{Derivative Estimate of Orthogonal Polynomials}

In the next few sections, we will prove the boundedness and small distance regularity of the density. Our proof follows the approach of [26] (cf: Lemma 3.3 and 3.4 in [26]), but the estimates are done in a different way due to the singularity of the potential. For the rest of this paper, it is convenient to rescale the local equilibrium measure to the interval $[-1,1]$ as we now explain.

Suppose $L \in \mathscr{G}$ and $\mathbf{y} \in \mathscr{Y}_{L}$. We change variables by introducing the transformation

$$
T: I_{\mathbf{y}} \rightarrow[-1,1], \quad \widetilde{w}=T(w):=\frac{2(w-\bar{y})}{\left|I_{\mathbf{y}}\right|}, \quad \text { with } \quad \bar{y}:=\frac{y_{-1}+y_{1}}{2}
$$

and its inverse

$$
w=T^{-1}(\widetilde{w})=\bar{y}+\frac{\widetilde{w}\left|I_{\mathrm{y}}\right|}{2},
$$

then $T\left(I_{\mathbf{y}}\right)=[-1,1]$. Let $\widetilde{\mu}_{\tilde{\mathbf{y}}}$ be the measure $\mu_{\mathbf{y}}^{(1)}$ (see $(7.5)$ ) rescaled to the interval $[-1,1]$, i.e.,

$$
\widetilde{\mu}_{\widetilde{\mathbf{y}}}(\mathrm{d} \widetilde{\mathbf{x}}):=\frac{1}{\widetilde{Z}_{n, \widetilde{\mathbf{y}}}} \exp \left[-n \sum_{i=1}^{n} U_{\widetilde{\mathbf{y}}}\left(\widetilde{x}_{i}\right)+2 \sum_{1 \leq i<j \leq n} \log \left|\widetilde{x}_{i}-\widetilde{x}_{j}\right|\right] \mathrm{d} \widetilde{\mathbf{x}}
$$


on $[-1,1]^{n}$ with

$$
U_{\widetilde{\mathbf{y}}}(\tilde{x}):=-\frac{2}{n} \sum_{|k|<n^{B}} \log \left|\widetilde{x}-\tilde{y}_{k}\right| .
$$

The $\ell$-point correlation functions of $\mu_{\mathbf{y}}$ and $\widetilde{\mu}_{\widetilde{\mathbf{y}}}$ are related by

$$
p_{n}^{(\ell)}\left(x_{1}, x_{2}, \ldots x_{n}\right)=p_{n}^{(\ell)}\left(\bar{y}+\frac{\tilde{x}_{1}\left|I_{\mathbf{y}}\right|}{2}, \ldots \bar{y}+\frac{\tilde{x}_{n}\left|I_{\mathbf{y}}\right|}{2}\right)=\left(\frac{2}{\left|I_{\mathbf{y}}\right|}\right)^{\ell} \widetilde{p}_{n}^{(\ell)}\left(\tilde{x}_{1}, \tilde{x}_{2}, \ldots \widetilde{x}_{n}\right) .
$$

Let $p_{j}(\lambda), j=0,1, \ldots$ denote the real orthonormal polynomials on $[-1,1]$ corresponding to the weight function $e^{-n U_{\tilde{y}}(\lambda)}$, i.e. $\operatorname{deg} p_{j}=j$ and

$$
\int_{-1}^{1} p_{j}(\lambda) p_{k}(\lambda) e^{-n U_{\tilde{y}}(\lambda)} \mathrm{d} \lambda=\delta_{j k}
$$

and define

$$
\psi_{j}(\lambda):=p_{j}(\lambda) e^{-n U_{\tilde{y}}(\lambda) / 2}
$$

to be orthonormal functions with respect to the Lebesgue measure on $[-1,1]$. Everything depends on $\mathbf{y}$, but $\mathbf{y}$ is fixed in this section and we will omit this dependence from the notation.

We define the $n$-th reproducing kernel

$$
K_{n}(\lambda, v)=\sum_{j=0}^{n-1} \psi_{j}(\lambda) \psi_{j}(v)
$$

that satisfies

$$
K_{n}(\lambda, v)=\int_{-1}^{1} K_{n}(\lambda, \zeta) K_{n}(\zeta, v) \mathrm{d} \zeta
$$

The density is given by

$$
\widetilde{\varrho}_{n}(\lambda)=\widetilde{p}_{n}^{(1)}(\lambda)=n^{-1} K_{n}(\lambda, \lambda)
$$

and the general $\ell$-point correlation function is given by

$$
\widetilde{p}_{n}^{(\ell)}\left(\lambda_{1}, \lambda_{2}, \ldots, \lambda_{\ell}\right)=\frac{(n-\ell) !}{n !} \operatorname{det}\left\{K_{n}\left(\lambda_{j}, \lambda_{k}\right)\right\}_{j, k=1}^{\ell}
$$

following the standard identities in orthogonal polynomials. For the rest of the paper we drop the tilde and all variables will denote the rescaled ones, i.e. all $x$ variables will be on the interval $[-1,1]$. All integrals in this section are understood on $[-1,1]$.

The basic ingredients of the approach [26] can be described as follows: Suppose that the following two properties hold for the normalized function $\psi=\psi_{j}, j=n-1, n$, and for some fixed $\kappa>0$

$$
\begin{gathered}
\int_{|x| \leq 1-\kappa / 2}\left|\psi^{\prime}(x)\right|^{2} \mathrm{~d} x \leq C n^{2+\bar{\varepsilon}} \\
n^{\delta} \int_{\left|x-x_{0}\right| \leq n^{-\delta}} \psi^{2}(x) \mathrm{d} x \leq C n^{\sigma}, \quad\left|x_{0}\right| \leq 1-\kappa
\end{gathered}
$$


for some positive $\sigma, \delta, \bar{\varepsilon}$ with $\sigma<1$. We will take take $\delta=1 / 4$, same as in [26]. Let

$$
\bar{\psi}=\frac{1}{2 \ell} \int_{\left|x-x_{0}\right| \leq \ell} \psi(x) \mathrm{d} x
$$

be the average of $\psi$ in the interval $\left|x-x_{0}\right| \leq \ell$ with some $x_{0},\left|x_{0}\right| \leq 1-\kappa$ and $\ell \leq \kappa / 2$. We have

$$
\left|\psi\left(x_{0}\right)\right| \leq|\bar{\psi}|+\left\|\psi^{\prime}\right\|_{L^{2}} \ell^{1 / 2} .
$$

Using (8.10) to estimate $|\bar{\psi}| \leq C \ell^{-1 / 2} n^{(\sigma-\delta) / 2}$ (under the assumption that $\ell<n^{-\delta}$ ) and using (8.9), we obtain

$$
\left|\psi\left(x_{0}\right)\right| \leq C \ell^{-1 / 2} n^{(\sigma-\delta) / 2}+C n^{1+\bar{\varepsilon} / 2} \ell^{1 / 2}
$$

Choosing $\ell=n^{-1+(\sigma-\delta-\bar{\varepsilon}) / 2}$ we have

$$
\left|\psi\left(x_{0}\right)\right| \leq n^{\frac{1}{2}+\frac{1}{4}(\sigma+\bar{\varepsilon}-\delta)} .
$$

Note that $\left|\psi\left(x_{0}\right)\right|=O\left(n^{\frac{1}{2}-\varepsilon^{\prime}}\right)$ with some $\varepsilon^{\prime}>0$ provided that $\sigma+\bar{\varepsilon}<\delta$. Suppose we can also prove that

$$
\left|\varrho^{\prime}(x)\right| \leq C n^{\varepsilon^{\prime \prime}}\left(\psi_{n-1}^{2}(x)+\psi_{n}^{2}(x)\right)
$$

with some small power $\varepsilon^{\prime \prime}$, then it will follow that $\left|\varrho^{\prime}(x)\right| \leq o(n)$ and this proves the regularity of the density over a distance of order $1 / n$. Together with the fact that the density is well approximated with the semicircle law on scales bigger than $1 / n$ this will show that the density is close to the semicirle law pointwise. In [26] the regularity of the density on larger scales followed from the smoothness of the potential (Theorem 2.2 of [26]). In our case this follows from (6.34) which is a consequence of the fact [15] that the semicircle law is precise on scales slightly larger than $1 / N$ that corresponds to scales bigger than $1 / n$ after rescaling.

In proving (8.9), (8.10) and 8.12 , one basic assumption in [26] requires the potential to be in $C^{2+v}$ for some $v>0$. The potential for our probability measure (8.2), parametrized by the boundary conditions $\mathbf{y}$, is singular near the boundary points $\{ \pm 1\}$. In order to control these singularities, besides using some special properties of orthogonal polynomials, we rely on [15] via 6.33) to provide essential estimates such as level repulsions. It turns out that we can only establish (8.9) and (8.10) for $\psi_{j}, j \leq n-1$ following this idea. The case of $j=n$ has to be treated completely differently. We now start to prove (8.9) for $\psi_{j}, j=n-1, n-2$.

Lemma 8.1. Suppose that $L \in \mathscr{G}, \mathbf{y} \in \mathscr{Y}_{L}$ and, after rescaling that sets $y_{-1}=-1, y_{1}=1$, let the y-configuration satisfy

$$
\sup _{|x| \leq 1} \sum_{1<|k|<n^{B}} \frac{1}{\left|x-y_{k}\right|} \leq \sum_{1<|k|<n^{B}}\left[\frac{1}{\left|y_{k}-y_{1}\right|}+\frac{1}{\left|y_{k}-y_{-1}\right|}\right] \leq C n^{1+3 \gamma}
$$

(note that the boundary terms $k= \pm 1$ are not included in the summations). Furthermore, assume that the density $\varrho_{n}$ satisfies

$$
\int_{-1+n^{-A}}^{1-n^{-A}}\left[(x+1)^{-2}+(1-x)^{-2}\right] \varrho_{n}(x) \mathrm{d} x \leq C n^{4 \gamma}
$$


for some $A \geq 60 B$. Then for the orthonormal functions $\psi_{j}$ from (8.4) we have

$$
\int_{-1}^{1} \psi_{j}^{2}(x)\left[\frac{1}{n} \sum_{|k|<n^{B}} \frac{1}{\left|x-y_{k}\right|}\right]^{2} \mathrm{~d} x \leq n^{6 \gamma} \quad j \leq n-1 .
$$

and

$$
\int_{-1}^{1}\left(\psi_{j}^{\prime}(x)\right)^{2} \mathrm{~d} x \leq C n^{2+6 \gamma} \quad j \leq n-1 .
$$

Notice that the assumptions $(8.13)$ and (8.14) follow from (6.31) and (6.33).

In this section and in the subsequent Sections 9 and 10 we work with orthogonal polynomials on $[-1,1]$ with respect to the potential $U_{\widetilde{\mathbf{y}}}(x)$ (see $(8.2)$ ). For brevity, we set $V(x)=U_{\widetilde{\mathbf{y}}}(x)$ in these three sections and we make the convention that the summation over the index $k$ that labels the elements of the external configuration $\mathbf{y}$ will always run over integers with for $1 \leq|k|<n^{B}$ unless otherwise indicated.

Proof. For simplicity, let $p(x)=p_{j}(x)$ and $\psi(x)=\psi_{j}(x)$. Then

$$
\int_{-1}^{1}\left(p^{\prime}(x)\right)^{2} e^{-n V(x)} \mathrm{d} x=\int_{-1}^{1}-p^{\prime \prime}(x) p(x) e^{-n V(x)} \mathrm{d} x+n \int_{-1}^{1} p^{\prime}(x) p(x) V^{\prime}(x) e^{-n V(x)} \mathrm{d} x .
$$

Note that $e^{-n V(x)}$ is zero at the boundary $x= \pm 1$ so the boundary term vanishes in the integration by parts. Since $p(x)$ is an orthogonal polynomial, it is orthogonal to all polynomials of lower degree, thus the first integral vanishes. By Schwarz inequality, the second integral is bounded by

$$
n \int_{-1}^{1} p^{\prime}(x) p(x) V^{\prime}(x) e^{-n V(x)} \mathrm{d} x \leq \frac{1}{2} \int_{-1}^{1}\left(p^{\prime}(x)\right)^{2} e^{-n V(x)} \mathrm{d} x+\frac{1}{2} \int p^{2}(x)\left(n V^{\prime}(x)\right)^{2} e^{-n V(x)} \mathrm{d} x
$$

We have thus proved that

$$
\int_{-1}^{1}\left(p^{\prime}(x)\right)^{2} e^{-n V(x)} \mathrm{d} x \leq 2 \int_{-1}^{1} p^{2}(x)\left(n V^{\prime}(x)\right)^{2} e^{-n V(x)} \mathrm{d} x .
$$

The last integral is bounded by

$$
\int_{-1}^{1} p^{2}(x)\left(n V^{\prime}(x)\right)^{2} e^{-n V(x)} \mathrm{d} x \leq I_{1}+I_{2}
$$

with

$$
I_{1}=2 \int_{-1}^{1}\left[\frac{1}{(x-1)^{2}}+\frac{1}{(x+1)^{2}}\right] \psi^{2}(x) \mathrm{d} x, \quad I_{2}=2 \int_{-1}^{1}\left[\sum_{k \neq \pm 1} \frac{1}{x-y_{k}}\right]^{2} \psi^{2}(x) \mathrm{d} x .
$$

From (8.13), and the normalization of $\psi$ we have

$$
I_{2} \leq C n^{2+6 \gamma} \text {. }
$$


To control the term $I_{1}$, we separate the integration regimes $|x \pm 1| \leq n^{-A}$ and $-1+n^{-A} \leq x \leq 1-n^{-A}$ for some big constant $A$. In the inside regime, we can use $|\psi(x)|^{2}=\left|\psi_{j}(x)\right|^{2} \leq n \varrho_{n}(x)$ since $j \leq n-1$. From (8.14) we obtain

$$
\int_{-1+n^{-A}}^{1-n^{-A}}\left[\frac{1}{(x-1)^{2}}+\frac{1}{(x+1)^{2}}\right] \psi^{2}(x) \mathrm{d} x \leq C n^{1+4 \gamma} .
$$

To estimate the singular part of the integral in $I_{1}$ near the boundary points, we can focus in estimating

the other endpoint being similar. Let

$$
\int_{-1}^{-1+n^{-A}} \frac{\psi^{2}(x)}{(1+x)^{2}} d x
$$

$$
g(x)=\frac{\psi(x)}{x+1} .
$$

Notice that $g(x)$ is a polynomial of degree $\operatorname{deg} g \leq 2 n^{2 B}+n$. From the Nikolskii inequality (see, e.g., Theorem A.4.4 of [5])

$$
\|g\|_{4} \leq C(\operatorname{deg} g)^{7.5}\|g\|_{1 / 4} \leq C n^{15 B}\|g\|_{1 / 4}
$$

with some universal constant $C$. Here $\|g\|_{p}$ is defined as $\left(\int_{-1}^{1}|g(x)|^{p} \mathrm{~d} x\right)^{1 / p}$ for any $0<p<\infty$. Notice that Nikolskii inequality holds between $L^{p}$ spaces even with exponents $p<1$. By the Hölder inequality,

$$
\begin{aligned}
\|g\|_{1 / 4}^{1 / 2}=\left(\int_{-1}^{1}|g(x)|^{1 / 4} \mathrm{~d} x\right)^{2} & \leq\left(\int_{-1}^{1}|g(x)|^{1 / 2}|x+1|^{1 / 2} \mathrm{~d} x\right)\left(\int_{-1}^{1}|x+1|^{-1 / 2} \mathrm{~d} x\right) \\
& \leq C\left(\int|g(x)|^{2}(x+1)^{2} \mathrm{~d} x\right)^{1 / 4} \\
& =C\|\psi\|_{2}^{1 / 2}=C .
\end{aligned}
$$

Thus from 8.22 ) we have $\|g\|_{4} \leq C n^{15 B}$ and by Hölder inequality we have

$$
\int_{-1}^{-1+n^{-A}} \frac{\psi^{2}(x)}{(x+1)^{2}} \mathrm{~d} x \leq C n^{-A / 2}\|g\|_{4}^{2} \leq C n^{30 B-A / 2} \leq C
$$

provided $A \geq 60 B$. Together with (8.21), this proves $I_{1} \leq C n^{1+4 \gamma}$. Combining this with 8.20 we obtain a bound $C n^{2+6 \gamma}$ for (8.18) which proves (8.15).

Using this estimate and (8.17) we obtain that

$$
\int_{-1}^{1}\left|p^{\prime}(x)\right|^{2} e^{-n V(x)} \mathrm{d} x \leq C n^{2+6 \gamma}
$$

Since

$$
\left|\psi^{\prime}(x)\right|^{2} \leq C\left[p^{\prime}(x)^{2}+p^{2}(x)\left(n V^{\prime}(x)\right)^{2}\right] e^{-n V(x)},
$$

we have thus proved that

$$
\int_{-1}^{1}\left|\psi^{\prime}(x)\right|^{2} \mathrm{~d} x \leq C n^{2+6 \gamma}+C \int_{-1}^{1} p^{2}(x)\left(n V^{\prime}(x)\right)^{2} e^{-n V(x)} \mathrm{d} x \leq C n^{2+6 \gamma}
$$

by using (8.18). This completes the proof. 


\section{Bound on smeared-out orthogonal polynomials}

Lemma 9.1. Let $\kappa, \delta_{0}>0$ be arbitrary positive numbers. Let $L \in \mathscr{G}, \mathbf{y} \in \mathscr{Y}_{L}$, suppose that the $\mathbf{y}$ configuration satisfies (8.13), (8.14) and the density $\varrho_{n}(x) \geq \delta_{0}>0$ for all $|x| \leq 1-\kappa$. Let $\psi=\psi_{n-1}$ or $\psi_{n-2}$ be an orthogonal function. Then we have

$$
n^{1 / 4} \int_{\left|x-x_{0}\right| \leq n^{-1 / 4}} \psi^{2}(x) \mathrm{d} x \leq C n^{3 \gamma}, \quad\left|x_{0}\right| \leq 1-\kappa
$$

with a constant $C$ depending on $\kappa$ and $\delta_{0}$.

Proof. For any $z=u+i \eta \in \mathbb{C}$ with $\eta>0$, let

$$
m_{n}(z)=\int_{-1}^{1} \frac{\varrho_{n}(x)}{x-z} \mathrm{~d} x
$$

denote the Stieltjes transform of the density and denote by

$$
G_{n}(x, y)=\widetilde{p}_{n}^{(2)}(x, y)-\widetilde{\varrho}_{n}(x) \widetilde{\varrho}_{n}(y)=-\frac{K_{n}(x, y)^{2}}{n(n-1)}+\frac{\widetilde{\varrho}_{n}(x) \widetilde{\varrho}_{n}(y)}{n-1}
$$

the truncated correlation function, where $\widetilde{p}_{n}^{(2)}$ was defined in (8.3) and computed from $(8.8)$. We will again drop the tilde in this proof.

We have the identity

$$
\int \frac{V^{\prime}(x) \varrho_{n}(x)}{x-z} \mathrm{~d} x=-\frac{n-1}{n} m_{n}^{2}(z)-\frac{1}{n} \int \frac{\varrho_{n}(x)}{(x-z)^{2}} \mathrm{~d} x-\frac{n-1}{n} \int \frac{G_{n}(x, y)}{(x-z)(y-z)} \mathrm{d} x \mathrm{~d} y .
$$

This identity follows from expressing $\varrho_{n}$ by an integral over $n-1$ variables of the equilibrium measure and then integrating by parts (see also (2.81) of [26]). Hence, by using (8.6), we have

$$
m_{n}^{2}(z)+\int \frac{V^{\prime}(x) \varrho_{n}(x)}{x-z} \mathrm{~d} x=-\frac{1}{2 n^{2}} \int K_{n}^{2}(x, y)\left(\frac{1}{x-z}-\frac{1}{y-z}\right)^{2} \mathrm{~d} x \mathrm{~d} y .
$$

The last integral can be bounded by

$$
\begin{aligned}
\left|\int K_{n}^{2}(x, y)\left(\frac{1}{x-z}-\frac{1}{y-z}\right)^{2} \mathrm{~d} x \mathrm{~d} y\right| & \leq\left|\int K_{n}^{2}(x, y) \frac{(x-y)^{2}}{(x-z)^{2}(y-z)^{2}} \mathrm{~d} x \mathrm{~d} y\right| \\
& \leq \eta^{-4} \int K_{n}^{2}(x, y)(x-y)^{2} \mathrm{~d} x \mathrm{~d} y \leq C \eta^{-4}
\end{aligned}
$$

where, to estimate the last integral, we have used the Christoffel-Darboux formula

$$
K_{n}(x, y)=J_{n-1} \frac{\psi_{n}(x) \psi_{n-1}(y)-\psi_{n}(y) \psi_{n-1}(x)}{x-y}, \quad J_{n-1}=\int_{-1}^{1} x \psi_{n-1}(x) \psi_{n}(x) \mathrm{d} x .
$$

We have thus proved that

$$
m_{n}^{2}(z)+\int \frac{V^{\prime}(x) \varrho_{n}(x)}{x-z} \mathrm{~d} x=O\left(n^{-2} \eta^{-4}\right), \quad z=u+i \eta
$$


We define a new measure $\mu_{\mathrm{y}}^{-}$on $[-1,1]^{n-1}$ as

$$
\mu_{\widetilde{\mathbf{y}}}^{-}\left(\mathrm{d} x_{1}, \ldots, \mathrm{d} x_{n-1}\right)=\frac{1}{Z_{\tilde{\mathbf{y}}, n}^{-}} \exp \left[-n \sum_{i=1}^{n-1} V\left(x_{i}\right)+2 \sum_{1 \leq i<j<n-1} \log \left|x_{i}-x_{j}\right|\right]
$$

where we already omitted the tildes and recall that $V(x)=U_{\mathbf{y}}(x)$. Note that this measure differs from (8.1) written in $n-1$ variables in that we kept the prefactor $n$ in front of the potential. Define

$$
\varrho_{n}^{-}(x)=\frac{n-1}{n} \int \mu_{\mathrm{y}}\left(x, \mathrm{~d} x_{2}, \mathrm{~d} x_{3}, \ldots \mathrm{d} x_{n-1}\right)
$$

and note that

$$
\varrho_{n}^{-}(x)=\frac{1}{n} \sum_{j=0}^{n-2} \psi_{j}^{2}(x)
$$

where $\psi_{j}$ 's are defined in (8.4). This latter formula follows from the recursive relation of the correlation functions for GUE-like ensembles, therefore

$$
\psi_{n-1}^{2}(x)=n\left(\varrho_{n}(x)-\varrho_{n}^{-}(x)\right)
$$

Let

$$
m_{n}^{-}(z)=\int_{-1}^{1} \frac{\varrho_{n}^{-}(x)}{x-z} \mathrm{~d} x
$$

be the Stieltjes transform of $\varrho_{n}^{-}$; then we have the analogue of 9.6

$$
\left[m_{n}^{-}(z)\right]^{2}+\int \frac{V^{\prime}(x) \varrho_{n}^{-}(x)}{x-z} \mathrm{~d} x=O\left(n^{-2} \eta^{-4}\right) .
$$

Subtracting this from $(9.6)$, we have

$$
n\left(m_{n}^{2}(z)-\left[m_{n}^{-}(z)\right]^{2}\right)=-\int \frac{V^{\prime}(x) \psi_{n-1}^{2}(x)}{x-z} \mathrm{~d} x+O\left(n^{-1} \eta^{-4}\right) .
$$

Assume that $u=\operatorname{Re} z$ satisfies $\left|u-x_{0}\right| \leq n^{-1 / 4}$. By adding $n\left(m_{n}(z)-m_{n}^{-}(z)\right) V^{\prime}(u)$ to the both sides of (9.7), we obtain

$$
n\left(m_{n}(z)-m_{n}^{-}(z)\right)\left(m_{n}(z)+m_{n}^{-}(z)+V^{\prime}(u)\right)=-\int \frac{\left(V^{\prime}(x)-V^{\prime}(u)\right) \psi_{n-1}^{2}(x)}{x-z} \mathrm{~d} x+O\left(n^{-1} \eta^{-4}\right) .
$$

We divide the integral into $\left|x-x_{0}\right| \leq v / 2$ and $\left|x-x_{0}\right| \geq v / 2$. In the first integration regime, since $\left|x_{0}\right| \leq 1-v$, we have

$$
\begin{aligned}
& \int_{\left|x-x_{0}\right| \leq v / 2}\left|\frac{V^{\prime}(x)-V^{\prime}(u)}{x-z}\right| \psi_{n-1}^{2}(x) \mathrm{d} x \\
& \quad \leq \sup _{\left|x-x_{0}\right| \leq v / 2} \frac{1}{n} \sum_{k} \frac{1}{|x-u|}\left|\frac{1}{y_{k}-x}-\frac{1}{y_{k}-u}\right| \int_{\left|x-x_{0}\right| \leq v / 2} \psi_{n-1}^{2}(x) \mathrm{d} x .
\end{aligned}
$$


Since $|x| \leq 1-v / 2,|u| \leq 1-v / 2$, we have $\left|y_{k}-u\right| \geq 2 v^{-1}$ for any $k$. Thus, by (8.13), the prefactor in (9.8) is bounded, uniformly in $|x| \leq 1-v / 2$, by

$$
\begin{gathered}
\frac{1}{n} \sum_{k} \frac{1}{|x-u|}\left|\frac{1}{y_{k}-x}-\frac{1}{y_{k}-u}\right| \leq \frac{1}{n} \sum_{k} \frac{1}{\left|y_{k}-x\right|\left|y_{k}-u\right|} \leq \frac{2}{v n} \sum_{k} \frac{1}{\left|y_{k}-x\right|} \\
\leq \frac{C}{n}\left[\frac{1}{|1-x|}+\frac{1}{|1+x|}+\sum_{k \neq \pm 1} \frac{1}{\left|y_{k}-y_{1}\right|}+\sum_{k \neq \pm 1} \frac{1}{\left|y_{k}-y_{-1}\right|}\right] \leq C n^{3 \gamma},
\end{gathered}
$$

where the constant $C$ depends on $v$ and we recall that $y_{-1}=-1, y_{1}=1$ in the rescaled variables. In the second integration regime we use $|x-u| \geq\left|x-x_{0}\right|-\left|x_{0}-u\right| \geq v / 4$ and obtain

$$
\begin{aligned}
\int_{\left|x-x_{0}\right| \geq v / 2} & \left|\frac{V^{\prime}(x)-V^{\prime}(u)}{x-z}\right| \psi_{n-1}^{2}(x) \mathrm{d} x \\
& \leq \frac{C}{n} \int_{\left|x-x_{0}\right| \geq v / 2} \sum_{k}\left|\frac{1}{y_{k}-x}-\frac{1}{y_{k}-u}\right| \psi_{n-1}^{2}(x) \mathrm{d} x \\
& \leq \frac{C}{n} \int \sum_{k}\left|\frac{1}{y_{k}-x}\right| \psi_{n-1}^{2}(x) \mathrm{d} x+\frac{C}{n} \sum_{k} \frac{1}{\left|y_{k}-u\right|} \leq C n^{3 \gamma}
\end{aligned}
$$

where we have used (8.15) and Hölder inequality to estimate the first term in the last line and using (8.13) for the second term.

We have thus proved that

$$
n\left|\left(m_{n}(z)-m_{n}^{-}(z)\right)\left(m_{n}(z)+m_{n}^{-}(z)+V^{\prime}(u)\right)\right| \leq n^{3 \gamma}+C n^{-1} \eta^{-4} .
$$

Hence

$$
n\left|m_{n}(z)-m_{n}^{-}(z)\right| \leq \frac{n^{3 \gamma}+C n^{-1} \eta^{-4}}{\operatorname{Im} m_{n}(z)}
$$

using that $\operatorname{Im} m_{n}^{-}(z)>0$. Since $\varrho_{n}(x) \geq \delta_{0}>0$ by assumption, $\operatorname{Im}_{n}(z)$ is bounded from below. Thus, choosing $\eta=n^{-1 / 4}$, we obtain

$$
\left|\int \frac{\psi_{n-1}^{2}(x)}{x-z} \mathrm{~d} x\right| \leq C n^{3 \gamma}
$$

with $C$ depending on $v$ and $\delta_{0}$. Taking imaginary part, we have

$$
\int \frac{\eta}{(x-u)^{2}+\eta^{2}} \psi_{n-1}^{2}(x) \mathrm{d} x \leq C n^{3 \gamma} .
$$

for any $u$ with $\left|u-x_{0}\right| \leq \eta=n^{-1 / 4}$. Integrating over $\left|u-x_{0}\right| \leq \eta$ and using

$$
\int_{\left|u-x_{0}\right| \leq \eta} \frac{\eta}{(x-u)^{2}+\eta^{2}} \geq c \cdot \mathbf{1}\left(\left|x-x_{0}\right| \leq \eta\right)
$$

with some positive constant $c$, we have proved (9.1) for $\psi=\psi_{n-1}$. The case $\psi=\psi_{n-2}$ can be done in a similar way. This completes the proof of Lemma 9.1 . 
Corollary 9.2. Suppose that the y-configuration satisfies (8.13), (8.14) and the density satisfies $\varrho_{n}(x) \geq \delta_{0}$ for all $|x| \leq 1-\kappa$ for some $\delta_{0}, \kappa>0$. Let $\psi=\psi_{j}$ with $j=n-2, n-1, n$ be an orthogonal function. Then

$$
\sup _{|x| \leq 1-\kappa}|\psi(x)|^{2} \leq C n^{1-\frac{1}{8}+11 \gamma}
$$

with a constant $C$ depending on $\kappa$ and $\delta_{0}$.

Proof. For the case $j=n-2, n-1$, the estimate (9.10), even with a better exponent, follows from the argument leading to (8.11) from the two assumptions (8.9) and (8.10) with $\delta=1 / 4, \bar{\varepsilon}=6 \gamma$ and $\sigma=3 \gamma$ :

$$
\sup _{|x| \leq 1-\kappa}\left|\psi_{j}(x)\right|^{2} \leq C n^{1-\frac{1}{8}+\frac{9}{2} \gamma} \quad j=n-2, n-1 .
$$

The estimate (8.9) was proven in Lemma 8.1, the estimate (8.10) follows from Lemma 9.1.

The proof of (9.10) for $\psi=\psi_{n}$ requires a different argument. Let $a_{j}$ be the leading coefficient of the (normalized) $j$-th orthogonal polynomial, i.e. $p_{j}(x)=a_{j} x^{j}+\ldots$ Observe that $p_{n}^{\prime}(x)=$ $n a_{n} x^{n-1}+\ldots=n\left(a_{n} / a_{n-1}\right) p_{n-1}(x)+\ldots$, where dots mean a polinomial of degree less than $n-1$. Thus

$$
\begin{aligned}
\frac{n a_{n}}{a_{n-1}} & =\int p_{n}^{\prime}(x) p_{n-1}(x) e^{-n V(x)} \mathrm{d} x \\
& =\int p_{n}(x) p_{n-1}^{\prime}(x) e^{-n V(x)} \mathrm{d} x+\int p_{n}(x) p_{n-1}(x) n V^{\prime}(x) e^{-n V(x)} \mathrm{d} x .
\end{aligned}
$$

The first integral on the right hand side vanishes. By the Schwarz inequality, we have

$$
\begin{aligned}
\frac{n\left|a_{n}\right|}{\left|a_{n-1}\right|} & \leq \int\left|p_{n}(x) p_{n-1}(x) n V^{\prime}(x)\right| e^{-n V(x)} \mathrm{d} x \\
& \leq\left[\int p_{n}^{2}(x) e^{-n V(x)} \mathrm{d} x\right]^{1 / 2}\left[\int\left|p_{n-1}(x) n V^{\prime}(x)\right|^{2} e^{-n V(x)} \mathrm{d} x\right]^{1 / 2} \leq C n^{1+3 \gamma},
\end{aligned}
$$

where the second integral was estimated in 8.15).

Recall the standard three-term recursion relation for orthogonal polynomials

$$
x p_{n-1}=a p_{n}+b p_{n-1}+c p_{n-2}
$$

with some real numbers $a, b, c$ depending on $n$. By comparing the leading coefficients, we have $a_{n-1}=a a_{n}$ and by orthonormality, we get

$$
a^{2}+b^{2}+c^{2}=\int_{-1}^{1} x^{2} p_{n-1}^{2}(x) e^{-n V(x)} \mathrm{d} x \leq 1 .
$$

In particular

$$
\frac{1}{|a|}=\left|\frac{a_{n}}{a_{n-1}}\right| \leq C n^{3 \gamma}
$$

from 9.12 . Hence, from 9.13,

$$
\left|p_{n}(x)\right| \leq\left|a^{-1}\left[(x-b) p_{n-1}(x)-c p_{n-2}(x)\right]\right| \leq C n^{3 \gamma}\left[\left|p_{n-1}(x)\right|+\left|p_{n-2}(x)\right|\right] .
$$

Using the bound (9.11), we obtain (9.10) for $\psi=\psi_{n}$ as well. 


\section{Regularity of Density}

Lemma 10.1. Let $L \in \mathscr{G}, \mathbf{y} \in \mathscr{Y}_{L}$. Suppose that the external y-configuration satisfies (8.13) and (8.14) and assume that $\gamma<\frac{1}{150}$. Then for any $\kappa>0$ we have

$$
\sup _{|x| \leq 1-\kappa}\left|\varrho_{n}^{\prime}(x)\right| \leq C n^{3 \gamma}\left(\psi_{n-1}^{2}(x)+\psi_{n}^{2}(x)+1\right) \leq C n^{1-\frac{1}{8}+14 \gamma}
$$

and

$$
\sup _{|x| \leq 1-2 \kappa}\left|\varrho_{n}(x)-\varrho_{s c}(\bar{y})\right| \leq C n^{-\gamma / 12}
$$

where the constant $C$ depends on $\kappa$.

Proof. The derivative of the density can be computed explicitly (see, e.g., (3.63) of [26]) as

$$
\varrho_{n}^{\prime}(x)=\int_{-1}^{1}\left[V^{\prime}(z)-V^{\prime}(x)\right] K_{n}^{2}(x, z) \mathrm{d} z
$$

In our case

$$
V^{\prime}(z)-V^{\prime}(x)=\frac{1}{n} \sum_{k}\left[\frac{1}{x-y_{k}}-\frac{1}{z-y_{k}}\right]
$$

and

$$
\frac{1}{x-y_{k}}-\frac{1}{z-y_{k}}=-\frac{x-z}{\left(x-y_{k}\right)^{2}}-\frac{(x-z)^{2}}{\left(z-y_{k}\right)\left(x-y_{k}\right)^{2}} .
$$

From the Christoffel-Darboux formula, we have

$$
\left|\int_{-1}^{1}(x-z)^{\alpha} K_{n}^{2}(x, z) \mathrm{d} z\right| \leq C\left(\psi_{n-1}^{2}(x)+\psi_{n}^{2}(x)\right), \quad \alpha=1,2 .
$$

Since $|x| \leq 1-\kappa$, we can estimate the contribution to (10.3) from the first term in (10.4) by

$$
\sum_{k} \frac{1}{n\left(x-y_{k}\right)^{2}}\left|\int_{-1}^{1}(x-z) K_{n}^{2}(x, z) \mathrm{d} z\right| \leq C n^{3 \gamma}\left(\psi_{n-1}^{2}(x)+\psi_{n}^{2}(x)\right),
$$

where we have used (8.13) to bound the factor in front of the integral

$$
\sup _{|x| \leq 1-\kappa} \sum_{k} \frac{1}{n\left(x-y_{k}\right)^{2}} \leq \sum_{k \neq \pm 1} \frac{1}{n \kappa\left|y_{-1}-y_{k}\right|}+\sum_{k \neq \pm 1} \frac{1}{n \kappa\left|y_{1}-y_{k}\right|}+\frac{C}{\kappa^{2} n} \leq C n^{3 \gamma} .
$$

The contribution from the second term in (10.4) is bounded by

$$
\begin{aligned}
\mid \frac{1}{n} \sum_{k} \frac{C}{\left(x-y_{k}\right)^{2}} & \int_{-1}^{1} \frac{(x-z)^{2}}{\left(z-y_{k}\right)} K_{n}^{2}(x, z) \mathrm{d} z \mid \\
& \leq C n^{3 \gamma} \kappa^{-2} \int_{-1}^{1} \frac{1}{n} \sum_{k} \frac{1}{\left|z-y_{k}\right|}\left[\psi_{n}(x) \psi_{n-1}(z)-\psi_{n}(z) \psi_{n-1}(x)\right]^{2} \mathrm{~d} z \\
& \leq C n^{3 \gamma} \sum_{j=0,1} \psi_{n-j}^{2}(x) \int_{-1}^{1} \frac{1}{n} \sum_{k} \frac{1}{\left|z-y_{k}\right|} \psi_{n+j-1}^{2}(z) \mathrm{d} z .
\end{aligned}
$$


The integral is estimated as

$$
\begin{aligned}
\int_{-1}^{1} \frac{1}{n} \sum_{k} \frac{1}{\left|z-y_{k}\right|} \psi_{n+j-1}^{2}(z) \mathrm{d} z \leq & \int_{-1}^{1} \frac{1}{n} \sum_{k \neq \pm 1}\left[\frac{1}{\left|y_{-1}-y_{k}\right|}+\frac{1}{\left|y_{1}-y_{k}\right|}\right] \psi_{n+j-1}^{2}(z) \mathrm{d} z \\
& +\int_{-1}^{1} \frac{1}{n} \sum_{k \pm 1}\left[\frac{1}{|1-z|}+\frac{1}{|1+z|}\right] \psi_{n+j-1}^{2}(z) \mathrm{d} z .
\end{aligned}
$$

The first term on the right hand side is bounded by $\mathrm{Cn}^{3 \gamma}$ using (8.13). In the second term, we split the integration into two regimes: $|z| \leq 1-n^{-A}$ and $1-n^{-A} \leq|z| \leq 1$ with some $A \geq 60 B$. In the first regime, we use the bound (9.10) to obtain $C A n^{-1 / 8+11 \gamma} \log n \leq C$ if $\gamma<\frac{1}{88}$. In the second regime we use the bound (8.23). This proves (10.1).

For the proof of (10.2) we use the derivative estimate and the fact that the density is close to the semicircle law on scale $n^{-1+\gamma}$ as given in (6.34). For any $x, y \in[-1+2 \kappa, 1-2 \kappa]$ we have

$$
\varrho(x)=\varrho(y)+\int_{x}^{y} \varrho^{\prime}(u) \mathrm{d} u
$$

Taking the average on the interval $I=\left[x-\frac{1}{2} n^{-1+\gamma}, x+\frac{1}{2} n^{-1+\gamma}\right]$, we get

$$
\left|\varrho(x)-n^{1-\gamma} \int_{I} \varrho(y) \mathrm{d} y\right| \leq n^{-1+\gamma}\left\|\varrho^{\prime}\right\|_{\infty} \leq C n^{-1 / 8+15 \gamma}
$$

Using (6.34), we have

$$
n^{1-\gamma} \int_{I} \varrho(y) \mathrm{d} y=\mathbb{E}_{\mu_{\mathrm{y}}} \frac{\mathscr{N}\left(I^{*}\right)}{N\left|I^{*}\right|}=\varrho_{s c}\left(T^{-1}(x)\right)+O\left(n^{-\gamma / 12}\right)=\varrho_{s c}(\bar{y})+O\left(n^{-\gamma / 12}\right)
$$

with $I^{*}:=T^{-1}(I)$, where we also used that

$$
\left|\varrho_{s c}\left(T^{-1}(x)\right)-\varrho_{s c}(\bar{y})\right| \leq\left|I_{\mathbf{y}}\right| \sup _{|x| \leq 2-\kappa}\left|\varrho^{\prime}(x)\right| \leq C n N^{-1} .
$$

Combining these inequalities, we arrive at $(10.2)$ and this completes the proof of Lemma 10.1

\section{Proof of the Main Theorem 2.1}

Let $V(x)=U_{\widetilde{\mathbf{y}}}(x)$ be the external potential on $I=[-1,1]$ given by 8.2 after rescaling. Notice that $V$ is continuous on $(-1,1)$ and $\lim _{|x| \rightarrow 1} V(x)=\infty$. Let $v(\mathrm{~d} x)$ be the equilibrium measure, defined as the unique solution to the variational problem

$$
\inf _{v \in \mathscr{M}^{1}}\left\{\int_{-1}^{1} \int_{-1}^{1} \log |s-t|^{-1} v(\mathrm{~d} s) v(\mathrm{~d} t)+\int_{-1}^{1} V(s) v(\mathrm{~d} s)\right\}
$$

where $\mathscr{M}^{1}$ is the space of probability measures on $[-1,1]$. For general properties of the equilibrium measure, see, e.g. Chapter 2 of [24] (and references therein) that specifically discusses the case 
of compact interval $I$ and continuous potential going to infinity at the endpoints. We point out however, that we follow the convention of [9] and [26] in what we call external potential; the potential in [24] and [23], denoted by $q(x)$ and $Q(x)$, respectively, differs by a factor of two from our convention: $q(x)=Q(x)=\frac{1}{2} V(x)$.

The equilibrium measure $v$ with support $S(v)$ satisfies the Euler-Lagrange equations

$$
\begin{array}{cc}
\int \log |s-t|^{-1} v(\mathrm{~d} s)+\frac{1}{2} V(t)=C & t \in S(v) \\
\int \log |s-t|^{-1} v(\mathrm{~d} s)+\frac{1}{2} V(t) \geq C & t \in I \backslash S(v)
\end{array}
$$

and $S(v) \subset(-1,1)$ (Theorem 2.1 of [24]). Moreover, since $V$ is convex in $(-1,1)$ such that $\lim _{|x| \rightarrow 1} V(x)=\infty$, the support $S(v)$ is an interval, $S(v)=[a, b]$, whose endpoints satisfy $-1<a<b<1$ and they are uniquely determined by the equations

$$
\int_{a}^{b} \frac{V^{\prime}(s) \mathrm{d} s}{\sqrt{(s-a)(b-s)}}=0, \quad \frac{1}{2 \pi} \int_{a}^{b} \frac{V^{\prime}(s) s \mathrm{~d} s}{\sqrt{(s-a)(b-s)}}=1 .
$$

according to Theorem 2.4 [24] (after adjusting a factor of 2).

In our case, the potential $V$ and thus the equilibrium measure $v$ depend on $n$ and the external configuration $\mathbf{y}$ in a non-trivial way. The main result of the recent work of Levin and Lubinsky [23] proves the universal sine-kernel behavior for the correlation function of the orthogonal polynomials with respect to a general $n$-dependent potential. This result fits exactly our situation, after the conditions of [23] are verified.

We recall the main result of [23] in a special form we will need.

Theorem 11.1. For each $n \geq 1$, consider a positive Borel measure $\mu_{n}$ on the real line whose $2 n+1$ moment is finite. Let $I=[-1,1]$ and assume that each $\mu_{n}$ is absolutely continuous on $I$ and they can be written as

$$
\mu_{n}(\mathrm{~d} x)=W_{n}^{2 n}(x) \mathrm{d} x
$$

where the non-negative functions $W_{n}$ are continuous on $I$. We define the potential $Q_{n}=-\log W_{n}: I \rightarrow$ $(-\infty,+\infty]$ and let $v_{n}$ be the solution of the variational problem (11.1) with $V=V_{n}=2 Q_{n}$. Let $J$ be a compact subinterval of $(-1,1)$. Assume the following conditions

(a) The equilibrium measure is absolutely continuous with $v_{n}(\mathrm{~d} x)=g_{n}(x) \mathrm{d} x$, where $g_{n}$ is positive and uniformly bounded in some open interval containing $J$;

(b) The family $\left\{Q_{n}^{\prime}\right\}_{n=1,2, \ldots}$ is equicontinuous and uniformly bounded in some open interval containing $J$;

(c) The density $\varrho_{n}(x)$ of the first $n$ orthogonal polynomials with respect to $\mu_{n}$ on I (defined in (8.7)) satisfies $C^{-1} \leq \varrho_{n}(x) \leq C$ in some open interval containing $J$;

(d) The following limit holds uniformly for $E \in J$ and $a$ in any fixed compact subset of $\mathbb{R}$ :

$$
\lim _{n \rightarrow \infty} \frac{\varrho_{n}(E)}{\varrho_{n}\left(E+\frac{x}{n}\right)}=1 .
$$


Then for the $n$-th reproducing kernel of the measure $\mu_{n}$ on I (defined in (8.5)) we have

$$
\lim _{n \rightarrow \infty} \frac{1}{n \varrho_{n}(E)} K_{n}\left(E+\frac{a}{n \varrho_{n}(E)}, E+\frac{b}{n \varrho_{n}(E)}\right)=\frac{\sin \pi(a-b)}{\pi(a-b)}
$$

uniformly for $E \in J$ and for $a, b$ in compact subsets of $\mathbb{R}$.

First we verify the conditions of this theorem for our case. We consider the sequence of measures $\mu_{n}$ on $\mathbb{R}$ that vanish outside of $I=[-1,1]$ and that are given by $\mu_{n}(\mathrm{~d} x)=e^{-n U_{\mathrm{y}}(x)} \mathrm{d} x$ on $I$, where $\mathbf{y} \in \mathscr{Y}_{L}$ is a sequence of good external configurations after rescaling for some $L \in \mathscr{G}$. Recall that the concept of good external configurations depends on $N$, i.e. $\mathscr{G}=\mathscr{G}_{N}$ and we recall the relation (4.15) between $n$ and $N$. We set $J=[-1+\sigma, 1-\sigma]$ for some $\sigma>0$. The measure $\mu_{n}$ is clearly absolutely continuous (actually it has a polynomial density), and since it is compactly supported, all moments are finite. Conditions (a) and (b) will be verified separately in Appendix F. Conditions (c) and (d) follow directly from (10.2) in Lemma 10.1 .

Now we start the proof of the main Theorem 2.1. Throughout this proof, $\mathbb{E}$ is the expectation for the Wigner ensemble with a small Gaussian component, i.e. $\mathbb{E}=\mathbb{E}_{f_{t}}$ with the earlier notation. All constants in this proof may depend on $\kappa$. We will use the results obtained in Sections 4410 , In these sections, various small exponents $\alpha, \beta, \gamma, \varepsilon$, and various large exponents $A, B$ need to be specified. The exponent $\beta$ is given in the theorem and it can be an arbitrary positive constant. The other exponents are determined in terms of $\beta$ subject to the following requirements: $\beta \geq 10 \varepsilon+\alpha$ (6.27), $\beta \geq(4 A+8) \varepsilon+\alpha$ (Lemma 6.1), $B \varepsilon<1 / 2$ (Section 7), $B \geq 20$ (Lemma 7.1) and $A \geq 60 B$ (Lemma 8.1). Finally, $\gamma \leq \frac{1}{10}$ can be an arbitrary positive number, independent of $\beta$. Obviously, these conditions can be simultaneously satisfied for any $\beta>0$ if $\alpha, \gamma, \varepsilon$ are chosen sufficiently small and $A, B$ sufficiently large. All constants in the proof depend on this choice.

Let $O(a, b)$ be a bounded function and $\delta<\kappa / 2$. In (2.9) we have to compute the limit of

$$
\begin{aligned}
\frac{1}{2 \delta} \int_{E_{0}-\delta}^{E_{0}+\delta} \mathrm{d} E & \int \mathrm{d} a \mathrm{~d} b \varrho_{s c}(E)^{-2} p_{N}^{(2)}\left(E+\frac{a}{N \varrho_{s c}(E)}, E+\frac{b}{N \varrho_{s c}(E)}\right) O(a, b) \\
& =\frac{N^{2}}{2 \delta} \int_{E_{0}-\delta}^{E_{0}+\delta} \mathrm{d} E \int \mathrm{d} u \mathrm{~d} v p_{N}^{(2)}(u, v) O\left((u-E) N \varrho_{s c}(E),(v-E) N \varrho_{s c}(E)\right) \\
& =\frac{N}{N-1} \frac{1}{2 \delta} \int_{E_{0}-\delta}^{E_{0}+\delta} \mathrm{d} E \mathbb{E} \sum_{j \neq k}^{N} O\left(\left(\lambda_{j}-E\right) N \varrho_{s c}(E),\left(\lambda_{k}-E\right) N \varrho_{s c}(E)\right), \\
& =: \frac{N}{N-1} T(N, \delta),
\end{aligned}
$$

where we have changed variables. Using the form of $O$ given in $(2.8)$, we have

$$
T(N, \delta)=\mathbb{E} \sum_{j \neq k}^{N} \frac{1}{2 \delta} \int_{E_{0}-\delta}^{E_{0}+\delta} \mathrm{d} E g\left(\left(\lambda_{j}-\lambda_{k}\right) N \varrho_{s c}(E)\right) h\left(\left(\frac{\lambda_{j}+\lambda_{k}}{2}-E\right) N \varrho_{s c}(E)\right) .
$$

We first show that

$$
\sup _{\delta \leq \kappa / 2} \sup _{N \in \mathbb{N}} T(N, \delta) \leq C
$$


with a constant depending on $\kappa$. To see this, let $R$ be a large number so that $g(x)=h(x)=0$ for $|x| \geq R$, then

$$
\begin{aligned}
T(N, \delta) & \leq C \mathbb{E} \sum_{j \neq k}^{N} \frac{1}{2 \delta} \int_{E_{0}-\delta}^{E_{0}+\delta} \mathrm{d} E \prod_{\ell=j, k} \mathbf{1}\left[\left|\lambda_{\ell}-E\right| \leq C R / N\right] \\
& \leq C \frac{1}{2 \delta} \int_{E_{0}-\delta}^{E_{0}+\delta} \mathrm{d} E \mathbb{E} \mathscr{N}^{2}[E-C R / N, E+C R / N] \leq C,
\end{aligned}
$$

where we have used that $\inf \left\{\varrho_{s c}(E):\left|E-E_{0}\right| \leq \delta\right\} \geq c>0$ and that

$$
\mathbb{E} \mathscr{N}_{I}^{k} \leq C_{k}(N|I|)^{k}
$$

for any interval $I$ of length $|I| \geq 1 / N$. The bound (11.8) follows from Eq. (3.11) in [15] after cutting the interval $I$ into subintervals of size $1 /(2 N)$.

The estimate (11.6) and similar ideas allow us to perform many cutoffs and approximations. For example, we can replace $\varrho_{s c}(E)$ in $g$ and $h$ by $\varrho:=\varrho_{s c}\left(E_{0}\right)$ in the definition of $T(N, \delta)$, see (11.5), at the expense of an error that vanishes in the limit $\delta \rightarrow 0$. We shall give a proof in case we perform the change for, say, $g$ :

$$
\begin{gathered}
\mathbb{E} \sum_{j \neq k}^{N} \frac{1}{2 \delta} \int_{E_{0}-\delta}^{E_{0}+\delta} \mathrm{d} E\left|g\left(\left(\lambda_{j}-\lambda_{k}\right) N \varrho_{s c}(E)\right)-g\left(\left(\lambda_{j}-\lambda_{k}\right) N \varrho_{s c}\left(E_{0}\right)\right)\right| h\left(\left(\frac{\lambda_{j}+\lambda_{k}}{2}-E\right) N \varrho_{s c}(E)\right) \\
\leq C \delta \mathbb{E} \sum_{j \neq k}^{N} \frac{1}{2 \delta} \int_{E_{0}-\delta}^{E_{0}+\delta} \mathrm{d} E \prod_{\ell=j, k} \mathbf{1}\left[\left|\lambda_{\ell}-E\right| \leq C R / N\right] \leq C \delta,
\end{gathered}
$$

where we used that $\varrho_{s c}^{\prime}(E)$ is uniformly bounded on $\left[E_{0}-\delta, E_{0}+\delta\right] \subset[-2+\kappa / 2,2-\kappa / 2]$. We will not repeat this type of simple argument in this proof.

After this replacement, we can perform the $\mathrm{d} E$ integration using that $\int h=1$ :

$$
\begin{aligned}
T(N, \delta) & =\mathbb{E} \sum_{j \neq k}^{N} g\left(\left(\lambda_{j}-\lambda_{k}\right) N \varrho\right) \frac{1}{2 \delta} \int_{E_{0}-\delta}^{E_{0}+\delta} \mathrm{d} E h\left(\left(\frac{\lambda_{j}+\lambda_{k}}{2}-E\right) N \varrho\right)+O(\delta) \\
& =\frac{1}{2 N \varrho \delta} \mathbb{E} \sum_{j \neq k}^{N} g\left(\left(\lambda_{j}-\lambda_{k}\right) N \varrho\right) \prod_{\ell=j, k} \mathbf{1}\left(\left|\lambda_{\ell}-E_{0}\right| \leq \delta\right)+O(\delta)+O\left(\delta^{-1} N^{-1}\right),
\end{aligned}
$$

where the last error comes from the contribution of eigenvalues within $C R / N$ distance to $E_{0} \pm \delta$. With the notation

$$
T^{*}(N, \delta):=\frac{1}{2 N \varrho \delta} \mathbb{E} \sum_{j \neq k}^{N} g\left(\left(\lambda_{j}-\lambda_{k}\right) N \varrho\right) \prod_{\ell=j, k} \mathbf{1}\left(\left|\lambda_{\ell}-E_{0}\right| \leq \delta\right),
$$

and using (11.4), we thus need to prove that

$$
\lim _{\delta \rightarrow 0} \lim _{N \rightarrow \infty} T^{*}(N, \delta)=\int g(a-b)\left[1-\left(\frac{\sin \pi(a-b)}{\pi(a-b)}\right)^{2}\right] \mathrm{d} a \mathrm{~d} b .
$$


Recall the definition of $\mathfrak{N}_{s c}(E)$ from (4.12) and its inverse function $\mathfrak{N}_{s c}^{-1}(E)$. Note that

$$
\left(\mathfrak{N}_{s c}^{-1}\right)^{\prime}(E) \leq C \kappa^{-1 / 2} \text { if } \quad-2+\kappa \leq E \leq 2-\kappa .
$$

We define

$$
\chi_{N, E_{0}, \delta}(j):=\mathbf{1}\left(M_{-} \leq j \leq M_{+}\right), \quad M_{ \pm}=N \cdot \mathfrak{N}_{s c}\left(E_{0} \pm \delta\right)
$$

and write

$$
\mathbf{1}\left(\left|\lambda_{j}-E_{0}\right| \leq \delta\right)=\chi_{N, E_{0}, \delta}(j)+U_{j}
$$

where $U_{j}$ is the error term, defined as the difference of $\mathbf{1}\left(\left|\lambda_{j}-E_{0}\right| \leq \delta\right)$ and $\chi_{N, E_{0}, \delta}(j)$. We thus have

$$
\begin{aligned}
T^{*}(N, \delta)= & \frac{1}{2 N \varrho \delta} \mathbb{E} \sum_{j \neq k}^{N} g\left(\left(\lambda_{j}-\lambda_{k}\right) N \varrho\right) \chi_{N, E_{0}, \delta}(j) \mathbf{1}\left(\left|\lambda_{k}-E_{0}\right| \leq \delta\right) \\
& +\frac{1}{2 N \varrho \delta} \mathbb{E} \sum_{j \neq k}^{N} g\left(\left(\lambda_{j}-\lambda_{k}\right) N \varrho\right) U_{j} \mathbf{1}\left(\left|\lambda_{k}-E_{0}\right| \leq \delta\right) .
\end{aligned}
$$

The last term is bounded by

$$
\left(\mathbb{E} \frac{1}{2 N \varrho \delta} \sum_{j}\left[\sum_{k: k \neq j}^{N} \mathbf{1}\left(\left|\lambda_{k}-E_{0}\right| \leq \delta\right) g\left(\left(\lambda_{j}-\lambda_{k}\right) N \varrho\right)\right]^{2} \mathbb{E}\left[\frac{1}{2 N \varrho \delta} \sum_{j} U_{j}^{2}\right]\right)^{1 / 2} .
$$

The first expectation is bounded by

$$
\frac{1}{2 N \varrho \delta} \mathbb{E} \sum_{k, k^{\prime}, j} \mathbf{1}\left(\left|\lambda_{k}-E_{0}\right| \leq \delta\right) \mathbf{1}\left(\left|\lambda_{k^{\prime}}-E_{0}\right| \leq \delta\right) \mathbf{1}\left(\left|\lambda_{j}-\lambda_{k}\right| \leq C / N\right) \mathbf{1}\left(\left|\lambda_{j}-\lambda_{k^{\prime}}\right| \leq C / N\right)
$$

Splitting the interval $\left[E_{0}-\delta-C / N, E_{0}+\delta+C / N\right]$ into overlapping subintervals $I_{\ell}$ of length $4 C / N$ with an overlap at least $2 C / N$, we get that this last expectation is bounded by

$$
\frac{1}{2 N \varrho \delta} \sum_{\ell} \mathbb{E} \mathscr{N}_{I_{\ell}}^{3} \leq C
$$

where we used the moment bound (11.8) with $k=3$ and the fact that the number of subintervals is $C N \delta$.

Since $\mathfrak{N}_{s c}$ is monotonic, the second expectation in 11.13 is bounded by

$$
\frac{1}{2 N \varrho \delta} \sum_{j} \mathbb{E}\left[\mathbf{1}\left(\left|\lambda_{j}-E_{0}\right| \leq \delta\right)-\mathbf{1}\left(\left|\mathfrak{N}_{s c}^{-1}(j / N)-E_{0}\right| \leq \delta\right)\right]^{2} .
$$

On the set $\Omega^{c}$ we estimate the difference of the two characteristic functions by 2 , and we get from (4.19) that the contribution is subexponentially small in $n$. On the set $\Omega$ we can use (4.21) and we see that the difference of the two characteristic functions can be nonzero only if

$$
\delta-C n^{-\gamma / 6} \leq\left|\mathfrak{N}_{s c}^{-1}(j / N)-E_{0}\right| \leq \delta+C n^{-\gamma / 6}
$$


i.e. the number of $j$ 's this can happen is bounded by $C N n^{-\gamma / 6}$. Recalling (4.15), we get

$$
\lim _{N \rightarrow \infty} \mathbb{E}\left[\frac{1}{2 N \varrho \delta} \sum_{j} U_{j}^{2}\right]=0
$$

therefore the second term in (11.12) vanishes in the $N \rightarrow \infty$ limit.

This shows that we can replace $\mathbf{1}\left(\left|\lambda_{j}-E_{0}\right| \leq \delta\right)$ by $\chi_{N, E_{0}, \delta}(j)$ in the definition of $T^{*}$ a with negligible error and we can do similarly for $k$ instead of $j$. Therefore, we need to prove that

$$
\lim _{\delta \rightarrow 0 N \rightarrow \infty} \frac{1}{2 N \varrho \delta} \mathbb{E} \sum_{\substack{M-\leq j, k \leq M_{+}, j \neq k}} g\left(\left(\lambda_{j}-\lambda_{k}\right) N \varrho\right)=\int g(a-b)\left[1-\left(\frac{\sin \pi(a-b)}{\pi(a-b)}\right)^{2}\right] \mathrm{d} a \mathrm{~d} b .
$$

and without loss of generality, we can assume that $g \geq 0$.

We define

$$
X_{L}:=n^{-1} \sum_{\substack{L \leq j, k \leq L+n \\ j \neq k}} g\left(\left(\lambda_{j}-\lambda_{k}\right) N \varrho\right)
$$

and let

$$
Q_{L}:=\mathbb{E} X_{L}
$$

We claim that

$$
\frac{1}{2 N \varrho \delta} \mathbb{E} \sum_{\substack{M_{-} \leq j, k \leq M_{+}, j \neq k}} g\left(\left(\lambda_{j}-\lambda_{k}\right) N \varrho\right)=\frac{1+O\left(n^{\gamma-1}\right)}{2 N \varrho \delta} \sum_{M_{-} \leq L \leq M_{+}} Q_{L}+O\left(N^{2} e^{-c n^{\gamma / 6}}\right) .
$$

To see this, we consider the expectation value separately on $\Omega$ and $\Omega^{c}$. Since the double sum contains at most $N^{2}$ terms and $\mathbb{P}\left(\Omega^{c}\right)$ is subexponentially small (4.19), it is sufficient to check (11.14) when the expectations are restricted to the set $\Omega$. On the set $\Omega$ we have

$$
\left(1-C n^{\gamma-1}\right) \sum_{M_{-} \leq L \leq M_{+}} X_{L} \leq \sum_{\substack{M_{-} \leq j, k \leq M_{+} \\ j \neq k}} g\left(\left(\lambda_{j}-\lambda_{k}\right) N \varrho\right) \leq\left(1+C n^{\gamma+1}\right) \sum_{M_{-} \leq L \leq M_{+}} X_{L},
$$

where $C$ depends on $\|g\|_{\infty}$. This follows from the fact that, by the support of $g$, only those $(j, k)$ index pairs give nonzero contribution for which $\left|\lambda_{j}-\lambda_{k}\right| \leq C / N$, and thus $|j-k| \leq C n^{\gamma}$ by (4.22). Therefore the sum $\sum_{L} X_{L}$ contains each pair $(j, k)$ at least $\left[n-C n^{\gamma}\right]$-times and at most $\left[n+C n^{\gamma}\right]$ times. Taking the expectation of (11.15) on $\Omega$, we obtain (11.14).

Since $Q_{L}$ is bounded by using (11.8), and

$$
\lim _{\delta \rightarrow 0} \lim _{N \rightarrow \infty} \frac{1}{2 N \varrho \delta} \sum_{M_{-} \leq L \leq M_{+}} 1=1,
$$

we only have to estimate $Q_{L}$ for a typical $L$. Additionally to $L \in\left\{M_{-}, M_{-}+1, \ldots, M_{+}\right\}$, we can thus assume that $L \in \mathscr{G}$, since the relative proportion of good indices approaches one within any index set with cardinality proportional with $N$ and which is away from the boundary (see (6.29)). More precisely, we fix two sequences $L_{-}(N)$ and $L_{+}(N)$ such that $L_{ \pm}(N) \in \mathscr{G}=\mathscr{G}_{N}$

$$
Q_{L_{-}(N)}=\min \left\{Q_{L}, L \in \mathscr{G}_{N}\right\}, \quad Q_{L_{+}(N)}=\max \left\{Q_{L}, L \in \mathscr{G}_{N}\right\}
$$


then it follows from (11.16) that

$$
\left(1-\varepsilon_{N, \delta}\right) Q_{L_{-}(N)} \leq \frac{1}{2 N \varrho \delta} \sum_{M_{-} \leq L \leq M_{+}} Q_{L} \leq\left(1+\varepsilon_{N, \delta}\right) Q_{L_{+}(N)}
$$

where $\lim _{\delta \rightarrow 0} \lim _{N \rightarrow \infty} \varepsilon_{N, \delta}=0$. We thus have to show that $Q_{L_{ \pm}(N)}$ converges to the sine kernel. We will actually prove that $Q_{L}$ converges to the sine-kernel for any sequence $L=L(N) \in \mathscr{G}=\mathscr{G}_{N}$. The dependence on $N$ will be omitted from the notation.

For $L \in \mathscr{G}$, we can compute the expectation as

$$
Q_{L}=\mathbb{E}_{f_{t}} \mathbb{E}_{f_{\mathbf{y}}} X_{L}=\mathbb{E} \mathbb{E}_{f_{\mathbf{y}}} X_{L}
$$

according to the convention that $\mathbb{E}=\mathbb{E}_{f_{t}}$. Recall that definition of the sets $\Omega_{1}=\Omega_{1}(L), \Omega_{2}=\Omega_{2}(L)$ and $\Omega_{3}(L)$ from (6.7), (6.15) and (6.20). Setting $\widetilde{\Omega}:=\Omega_{1} \cap \Omega_{2} \cap \Omega_{3}$, we see that the probability of its complement is $\mathbb{P}\left(\widetilde{\Omega}^{c}\right) \leq C n^{-2}$ (see (6.8), (6.16) and (6.21)). Since $X_{L} \leq C n$, we only have to consider external configurations such that $\mathbf{y} \in \widetilde{\Omega}$. Thus

$$
\begin{aligned}
Q_{L} & =\mathbb{E} \mathbf{1}(\mathbf{y} \in \widetilde{\Omega}) \mathbb{E}_{f_{\mathbf{y}}} X_{L}+O\left(n^{-1}\right) \\
& =\mathbb{E} \mathbf{1}(\mathbf{y} \in \widetilde{\Omega})\left[\mathbb{E}_{\mu_{\mathbf{y}}} X_{L}+\int\left(f_{\mathbf{y}}-1\right) X_{L} \mathrm{~d} \mu_{\mathbf{y}}\right]+O\left(n^{-1}\right) .
\end{aligned}
$$

The second term in the square bracket will be an error term since it is bounded by

$$
\mathbb{E} \mathbf{1}(\mathbf{y} \in \widetilde{\Omega}) \int\left|f_{\mathbf{y}}-1\right|\left|X_{L}\right| \mathrm{d} \mu_{\mathbf{y}} \leq C n \mathbb{E} \mathbf{1}(\mathbf{y} \in \widetilde{\Omega}) \int\left|f_{\mathbf{y}}-1\right| \mathrm{d} \mu_{\mathbf{y}}
$$

Since $\mathbf{y} \in \widetilde{\Omega}$ and $L \in \mathscr{G}$, we have

$$
\int\left|f_{\mathbf{y}}-1\right| \mathrm{d} \mu_{\mathbf{y}} \leq n^{-2}
$$

from (6.26) and (6.27) and we thus obtain

$$
Q_{L}=\mathbb{E} \mathbf{1}(\mathbf{y} \in \widetilde{\Omega}) \mathbb{E}_{\mu_{\mathrm{y}}} X_{L}+O\left(n^{-1}\right)
$$

For the main term, by using (7.6) and assuming that $B$ is large enough, we can also replace the measure $\mu_{\mathbf{y}}$ by its cutoff version $\mu_{\mathbf{y}}^{(1)}$ with a negligible error. Let $\varrho_{\mathbf{y}}=p_{\mathbf{y}}^{(1)}:=p_{\mu_{\mathbf{y}}^{(1)}}^{(1)}$ denote the density and $p_{\mathbf{y}}^{(2)}:=p_{\mu_{\mathbf{y}}^{(1)}}^{(2)}$ denote the two point marginal of this measure. Thus we have

$$
Q_{L}=(n-1) \mathbb{E} \mathbf{1}(\mathbf{y} \in \widetilde{\Omega}) \int_{y_{-1}}^{y_{1}} \mathrm{~d} \alpha \int_{y_{-1}}^{y_{1}} \mathrm{~d} \beta p_{\mathbf{y}}^{(2)}(\alpha, \beta) g((\alpha-\beta) N \varrho)+O\left(n^{-1}\right) .
$$

Since $\mu_{\mathbf{y}}^{(1)}$ is an equilibrium measure, its correlation functions can be obtained as determinants of the appropriate $K$ kernels, see 8.8 . In particular

$$
0 \leq p_{\mathbf{y}}^{(2)}(u, v)=\frac{n-1}{n} p_{\mathbf{y}}^{(1)}(u) p_{\mathbf{y}}^{(1)}(v)-\frac{1}{n(n-1)} K^{2}(u, v) \leq \varrho_{\mathbf{y}}(u) \varrho_{\mathbf{y}}(v)
$$


holds for the marginals of the measure $\mu_{\mathbf{y}}^{(1)}$. The lower bound on $p^{(2)}$ follows from the fact that $K$ is the kernel of a positive operator, i.e. $|K(u, v)|^{2} \leq K(u, u) K(v, v)$.

Let $0<\kappa \leq 1 / 10$. We now show that, up to an error of order $\kappa$, the $\mathrm{d} \alpha$ integration in (11.18) can be restricted from $I_{\mathbf{y}}=\left[y_{-1}, y_{1}\right]$ onto

$$
I_{\mathbf{y}}^{*}=\left[y_{-}^{*}, y_{+}^{*}\right]:=\left[\bar{y}-\frac{1-2 \kappa}{2}\left|I_{\mathbf{y}}\right|, \bar{y}+\frac{1-2 \kappa}{2}\left|I_{\mathbf{y}}\right|\right], \quad \bar{y}=\frac{y_{-1}+y_{1}}{2},
$$

i.e. onto an interval in the middle of $I_{\mathrm{y}}$ with length $(1-4 \kappa)\left|I_{\mathrm{y}}\right|$. Similarly, the $\mathrm{d} \beta$ integration will be restricted to

$$
I_{\mathbf{y}}^{* *}=\left[y_{-}^{* *}, y_{+}^{* *}\right]:=\left[\bar{y}-\frac{1-\kappa}{2}\left|I_{\mathrm{y}}\right|, \bar{y}+\frac{1-\kappa}{2}\left|I_{\mathbf{y}}\right|\right], \quad \bar{y}=\frac{y_{-1}+y_{1}}{2},
$$

i.e. onto an interval in the middle of $I_{\mathrm{y}}$ with length $(1-2 \kappa)\left|I_{\mathrm{y}}\right|$. We show how to restrict the $\mathrm{d} \alpha$ integration, the other one is analogous.

The difference between the full $\mathrm{d} \alpha$ integral and the restricted one is given by

$$
C n \mathbb{E} \mathbf{1}(\mathbf{y} \in \widetilde{\Omega}) \int_{I_{\mathbf{y}} \backslash I_{\mathbf{y}}^{*}} \mathrm{~d} \alpha \int_{y_{-1}}^{y_{1}} \mathrm{~d} \beta p_{\mathbf{y}}^{(2)}(\alpha, \beta) g((\alpha-\beta) N \varrho) .
$$

To do this estimate, we go back from the equilibrium measure $\mu_{\mathbf{y}}^{(1)}$ to $f_{\mathbf{y}}$ and we also remove the constraint $\widetilde{\Omega}$. As above, all these changes result in negligible errors. Moreover, we can insert $\Omega$ at the expense of a negligible error since $\mathbb{P}(\Omega)$ is subexponentially small. Thus $(11.20)$ can be estimated by

$$
\frac{C}{n} \mathbb{E} \mathbf{1}_{\Omega} \sum_{\substack{L \leq j, k \leq L+n \\ j \neq k}} g\left(\left(\lambda_{j}-\lambda_{k}\right) N \varrho\right)\left[\mathbf{1}\left(\lambda_{j}-\lambda_{L} \leq 2 \kappa\left(\lambda_{L+n}-\lambda_{L}\right)\right)+\mathbf{1}\left(\lambda_{j}-\lambda_{L} \geq(1-2 \kappa)\left(\lambda_{L+n}-\lambda_{L}\right)\right)\right]
$$

up to negligible errors. On the set $\Omega$ we know from (4.22) that

$$
N \varrho\left(\lambda_{L+n}-\lambda_{L}\right)=n+O\left(n^{4 / 5}\right), \quad N \varrho\left(\lambda_{j}-\lambda_{L}\right)=(j-L)+O\left(n^{4 / 5}\right)
$$

assuming that $\gamma \leq 1 / 20$. Thus the first term in the square bracket of $(11.20)$ can be estimated by

$$
C n^{-1} \mathbb{E} \mathbf{1}_{\Omega} \sum_{L \leq k \leq L+n} \sum_{j \neq k} \mathbf{1}\left(L \leq j \leq L+2 \kappa n+C n^{4 / 5}\right) g\left(\left(\lambda_{j}-\lambda_{k}\right) N \varrho\right) \leq C \kappa
$$

taking into account (11.8) as before. Similar estimate holds for the second term in (11.21). Thus, restricting the $\mathrm{d} \alpha$-integration to $I_{\mathbf{y}}^{*}$ results in an error of order $O(\kappa)$.

Doing the same restriction for the $\mathrm{d} \beta$ integral, we can from now on assume that both integrations in (11.18) are restricted to $I_{\mathrm{y}}^{*}$, i.e. it is separated away from the boundary. In particular, from (10.2) and after rescaling, we know that $\varrho_{\mathrm{y}}(\alpha)$ and $\varrho_{\mathrm{y}}(\beta)$ are essentially constant and equal to $\left|I_{\mathrm{y}}\right|^{-1}\left(1+O\left(n^{-\gamma / 12}\right)\right.$. Moreover, on the set $\widetilde{\Omega}$, we know from 6.13 ) that $\left|I_{\mathrm{y}}\right|^{-1}=\frac{N \varrho}{n}\left(1+O\left(n^{\gamma-1 / 4}\right)\right)$, i.e.

$$
\varrho_{\mathbf{y}}(\beta)=\frac{N \varrho}{n}\left(1+O\left(n^{-\gamma / 12}\right)\right), \quad \text { for any } \beta \in I_{\mathbf{y}}^{* *}
$$


Since $I_{\mathrm{y}}^{*} \subset I_{\mathbf{y}}^{* *}$, the same formula holds for $\varrho_{\mathbf{y}}(\alpha)$ for all $\alpha \in I_{\mathbf{y}}^{*}$.

We now compute the restricted integrals in (11.18). Changing variables from $\beta$ to $b$ with $\beta=$ $\alpha+b\left(n \varrho_{\mathbf{y}}(\alpha)\right)^{-1}$, we have

$$
\begin{aligned}
Q_{L}= & \mathbb{E} \mathbf{1}(\mathbf{y} \in \widetilde{\Omega}) \int_{y_{-}^{*}}^{y_{+}^{*}} \mathrm{~d} \alpha \frac{n-1}{n \varrho_{\mathbf{y}}(\alpha)} \int_{\left(y_{-}^{* *}-\alpha\right) \varrho_{\mathbf{y}}(\alpha)}^{\left(y_{+}^{* *}-\alpha\right) \varrho_{\mathbf{y}}(\alpha)} \mathrm{d} b p_{\mathbf{y}}^{(2)}\left(\alpha, \alpha+\frac{b}{n \varrho_{\mathbf{y}}(\alpha)}\right) g\left(\frac{-N \varrho b}{n \varrho_{\mathbf{y}}(\alpha)}\right) \\
& +O\left(n^{-1}\right)+O(\kappa) .
\end{aligned}
$$

Since $g$ is smooth and has compact support, we have

$$
g\left(\frac{-N \varrho b}{n \varrho_{\mathbf{y}}(\alpha)}\right)=g(-b)+\xi, \quad|\xi| \leq C\left|\frac{N \varrho}{n \varrho_{\mathbf{y}}(\alpha)}-1\right| \leq C n^{-\gamma / 12}
$$

from (11.23). Therefore, when we insert (11.25) into (11.24) and use (11.19), the error term involving $\xi$ is bounded by

$$
\begin{aligned}
C \mathbb{E} \mathbf{1}(\mathbf{y} \in & \widetilde{\Omega}) \int_{I_{\mathbf{y}}^{*}} \mathrm{~d} \alpha \frac{1}{\varrho_{\mathbf{y}}(\alpha)} \int_{\left(y_{-}^{* *}-\alpha\right) n \varrho_{\mathbf{y}}(\alpha)}^{\left(y_{+}^{* *}-\alpha\right) \varrho_{\mathbf{y}}(\alpha)} \mathrm{d} b p_{\mathbf{y}}^{(2)}\left(\alpha, \alpha+\frac{b}{n \varrho_{\mathbf{y}}(\alpha)}\right)\left|\frac{N \varrho}{n \varrho_{\mathbf{y}}(\alpha)}-1\right| \\
& \leq C n^{-\gamma / 12} \mathbb{E} \mathbf{1}(\mathbf{y} \in \widetilde{\Omega}) \int_{I_{\mathbf{y}}^{*}} \mathrm{~d} \alpha \int_{\left(y_{-}^{* *}-\alpha\right) n \varrho_{\mathbf{y}}(\alpha)}^{\left(y_{+}^{* *}-\alpha\right) n \varrho_{\mathbf{y}}(\alpha)} \mathrm{d} b \varrho_{\mathbf{y}}\left(\alpha+\frac{b}{n \varrho_{\mathbf{y}}(\alpha)}\right) \\
& \leq C n^{-\gamma / 12} \mathbb{E} \mathbf{1}(\mathbf{y} \in \widetilde{\Omega}) \int_{I_{\mathbf{y}}^{*}} \varrho_{\mathbf{y}}(\alpha) \mathrm{d} \alpha \int_{I_{\mathbf{y}}^{* *}} \varrho_{\mathbf{y}}(\beta) \mathrm{d} \beta \\
& \leq C n^{-\gamma / 12}
\end{aligned}
$$

using that, by definition,

$$
\int_{I_{\mathrm{y}}^{*}} \varrho_{\mathrm{y}}(\alpha) \mathrm{d} \alpha \leq \int_{I_{\mathrm{y}}} \varrho_{\mathrm{y}}(\alpha) \mathrm{d} \alpha=1
$$

and similar bound holds for the $\beta$-integral.

Thus we can replace the variable of $g$ in $(11.24)$ by $-b$ with negligible errors. Now Theorem 11.1 states that

$$
\frac{1}{\varrho_{\mathbf{y}}(\alpha)^{2}} p_{\mathbf{y}}^{(2)}\left(\alpha, \alpha+\frac{b}{n \varrho_{\mathbf{y}}(\alpha)}\right) \rightarrow\left[1-\left(\frac{\sin \pi b}{\pi b}\right)^{2}\right]
$$

Clearly, as $n \rightarrow \infty$,

$$
\left(y_{ \pm}^{* *}-\alpha\right) n \varrho_{\mathbf{y}}(\alpha) \rightarrow \pm \infty
$$

for all $\alpha \in I_{\mathrm{y}}^{*}$, i.e. the integration limits can be extended to infinity, noting that $g$ is compactly supported. Finally, from (11.23) we have

$$
\int_{I_{\mathrm{y}}^{*}} \varrho_{\mathrm{y}}(\alpha) \mathrm{d} \alpha \geq 1-O(\kappa)-O\left(n^{\gamma-1}\right)
$$

Combining all these estimates with Theorem 11.1, we obtain

$$
Q_{L}=\int_{-\infty}^{\infty} \mathrm{d} b\left[1-\left(\frac{\sin \pi b}{\pi b}\right)^{2}\right] g(-b)+O\left(n^{-\gamma / 12}\right)+O(\kappa)+o(1),
$$


where the last term error term is from Theorem 11.1 that goes to zero as $N \rightarrow \infty$. Taking the $N \rightarrow \infty, \delta \rightarrow 0$ and $\kappa \rightarrow 0$ limits in this order, we arrive at the proof of Theorem 2.1 .

\section{A Proof of Theorem 4.1}

We start with the proof of (4.4) and (4.5). From Theorem 4.6 of [15], we have

$$
\mathbb{P}(|m(x+i y)| \geq K) \leq C e^{-c \sqrt{K N|y|}}
$$

for all $K>0$ sufficiently large, and $|y| \geq(\log N)^{4} / N$. Since moreover $|m(x+i y)| \leq|y|^{-1}$ with probability one, we obtain, under the assumption $N|y| \geq(\log N)^{4}$,

$$
\mathbb{E}|m(x+i y)|^{q} \leq K^{q}+C|y|^{-q} e^{-c \sqrt{K N|y|}} \leq C_{q}
$$

uniformly in $N, x$. The bound (4.5) follows because $\omega_{y}(x)=\pi^{-1} \operatorname{Im} m(x+i y)$.

To prove the results about the closeness of $m(z)$ or $\mathbb{E} m(z)$ to $m_{s c}(z)$, we first recall the key identity about the trace of a resolvent in terms of resolvents of minors (see, e.g., (4.5) of [13]):

$$
m(z)=\frac{1}{N} \operatorname{Tr} \frac{1}{H-z}=\frac{1}{N} \sum_{k=1}^{N} \frac{1}{-m(z)-z+\delta_{k}(z)}
$$

with

$$
\delta_{k}(z)=h_{k k}+m(z)-\left(1-\frac{1}{N}\right) m^{(k)}(z)-X^{(k)}(z)
$$

and

$$
m^{(k)}(z)=\frac{1}{N-1} \operatorname{Tr} \frac{1}{B^{(k)}-z}, \quad X^{(k)}(z)=\frac{1}{N} \sum_{\alpha} \frac{\xi_{\alpha}^{(k)}-1}{\lambda_{\alpha}^{(k)}-z}, \quad \xi_{\alpha}^{(k)}=N\left|\mathbf{a}^{(k)} \cdot \mathbf{v}_{\alpha}^{(k)}\right|^{2} .
$$

Here $B^{(k)}$ is the $(k k)$-minor of $H$ (the $(N-1) \times(N-1)$ matrix obtained by removing the $k$-th row and the $k$-th column from $H), \lambda_{\alpha}^{(k)}, \mathbf{v}_{\alpha}^{(k)}$ are the eigenvalues and the eigenvectors of $B^{(k)}$, and $\mathbf{a}^{(k)}=$ $\left(h_{k 1}, \ldots, h_{k, k-1}, h_{k, k+1}, \ldots h_{k N}\right)$. Throughout the proof we let $x, y$ denote the real and imaginary parts of $z=x+i y$. Moreover, we will restrict our attention to $y>0$. The case $y<0$ can be handled similarly.

Step 1. Lower bound on $|m(z)+z|$. There exist constants $C, c>0$ such that

$$
\mathbb{P}(|m(x+i y)+(x+i y)| \leq c) \leq e^{-C \sqrt{N y}}
$$

for all $x \in \mathbb{R}, y \geq(\log N)^{4} / N$, and for all $N$ large enough (depending only on the choice of $C, c$ ). 
To show (A.3), we use a continuity argument. We claim that there exist positive constants $C_{1}, C_{2}, C_{3}, c>0$ such that the following four conditions are satisfied:

$$
\begin{aligned}
& \inf _{z \in \mathbb{C} \backslash[-2,2]}\left|z+m_{\mathrm{sc}}(z)\right| \geq 2 c \\
& \mathbb{P}\left(|m(x+i y)| \geq \frac{1}{2 c}\right) \leq \frac{e^{-C_{1} \sqrt{N y}}}{3} \quad \text { for all } x \in \mathbb{R}, y \geq(\log N)^{4} / N \\
& \mathbb{P}\left(\sup _{1 \leq k \leq N}\left|\delta_{k}(x+i y)\right| \geq \frac{c}{16}\right) \leq \frac{e^{-C_{2} \sqrt{N y}}}{3} \quad \text { for all } x \in \mathbb{R}, y \geq(\log N)^{4} / N \\
& \mathbb{P}\left(\left|m(x+i y)-m_{\mathrm{sc}}(x+i y)\right| \geq c\right) \leq \frac{e^{-C_{3} \sqrt{N y}}}{3} \quad \text { for all }|x| \leq 1, y \geq(\log N)^{4} / N
\end{aligned}
$$

The first condition can be checked explicitly from (4.3). The second condition follows from the upper bound in Theorem 4.6 of [15]. The third condition can be satisfied because of Lemma 4.2 in [15], combined with the fact that $\mathbb{P}\left(\max _{k}\left|h_{k k}\right| \leq(c / 48)\right) \leq e^{-C N}$ and with the observation that

$$
\left|m(z)-\left(1-\frac{1}{N}\right) m^{(k)}(z)\right| \leq \frac{C}{N y}
$$

with probability one (see, for example (2.7) in [14]). Finally, the last condition can be verified by Theorem 4.1 of [15]. Note that the last three conditions only need to hold for all $N>N_{0}\left(c, C_{1}, C_{2}, C_{3}\right)$ large enough. Fix $C=\min \left(C_{1}, C_{2}, C_{3}\right)$.

For $|x| \leq 1, y \geq(\log N)^{4} / N$ we have (using the first and the last equation in (A.4))

$$
\mathbb{P}(|m(x+i y)+(x+i y)| \leq c) \leq \mathbb{P}\left(\left|m(x+i y)-m_{\mathrm{sc}}(x+i y)\right| \geq c\right) \leq e^{-c \sqrt{N y}} .
$$

Hence (A.3) holds true (with the defined constants $c, C$ ) for every $|x| \leq 1, y \geq(\log N)^{4} / N$. Suppose now that $(A .3)$ holds for a given $z=x+i y \in \mathbb{C}$. Then we claim that (A.3) holds true for all

$$
z^{\prime}=x^{\prime}+i y^{\prime} \in B_{z}=\left\{z^{\prime} \in \mathbb{C}:\left|z-z^{\prime}\right| \leq D N^{-2}, \quad \operatorname{Im} z^{\prime} \geq(\log N)^{4} / N\right\}
$$

for a constant $D$ depending only on $c$, and for all $N>N_{0}$; this implies immediately that (A.3) holds true for all $x \in \mathbb{R}, y \geq(\log N)^{4} / N$ and $N>N_{0}$.

To prove (A.3) for $z^{\prime} \in B_{z}$, notice that $\left|m^{\prime}(z)\right| \leq N^{2}$ for all $z \in \mathbb{C}$ with $\operatorname{Im} z \geq(\log N)^{4} / N$ with probability one. Therefore, using (A.3) for $z$, we find that

$$
\mathbb{P}\left(\left|m\left(z^{\prime}\right)+z^{\prime}\right| \leq \frac{c}{2}\right) \leq e^{-C \sqrt{N y^{\prime}}}
$$

for all $z^{\prime} \in B_{z}$. Expanding (A.1), we obtain that

$$
m\left(z^{\prime}\right)+\frac{1}{m\left(z^{\prime}\right)+z^{\prime}}=-\frac{1}{N} \sum_{k=1}^{N} \frac{1}{m\left(z^{\prime}\right)+z^{\prime}} \delta_{k}\left(z^{\prime}\right) \frac{1}{m\left(z^{\prime}\right)+z^{\prime}-\delta_{k}\left(z^{\prime}\right)} .
$$


Therefore,

$$
\begin{aligned}
\mathbb{P}\left(\frac{1}{\left|m\left(z^{\prime}\right)+z^{\prime}\right|} \geq \frac{1}{c}\right) & \leq \mathbb{P}\left(\left|m\left(z^{\prime}\right)\right| \geq \frac{1}{2 c}\right)+\mathbb{P}\left(\frac{1}{N} \sum_{k=1}^{N} \frac{\left|\delta_{k}\left(z^{\prime}\right)\right|}{\left|m\left(z^{\prime}\right)+z^{\prime}\right|\left|m\left(z^{\prime}\right)+z^{\prime}-\delta_{k}\left(z^{\prime}\right)\right|} \geq \frac{1}{2 c}\right) \\
& \leq \mathbb{P}\left(\left|m\left(z^{\prime}\right)\right| \geq \frac{1}{2 c}\right)+\mathbb{P}\left(\left|m\left(z^{\prime}\right)+z^{\prime}\right| \leq \frac{c}{2}\right)+\mathbb{P}\left(\sup _{1 \leq k \leq N}\left|\delta_{k}\left(z^{\prime}\right)\right| \geq \frac{c}{16}\right) \\
& \leq e^{-C \sqrt{N y}}
\end{aligned}
$$

where we used (A.4) and (A.6). This implies A.3) for $z^{\prime} \in B_{z}$, and completes the proof of Step 1.

Step 2. Convergence to the semicircle in probability. Suppose that $|x| \leq K,(\log N)^{4} / N \leq y \leq 1$. Then there exist constants $c, C, \delta_{0}$, only depending on $K$, such that

$$
\mathbb{P}\left(\left|m(x+i y)-m_{\mathrm{sc}}(x+i y)\right| \geq \delta\right) \leq C e^{-c \delta \sqrt{N y|2-| x||}}
$$

for all $\delta<\delta_{0}$, and all $N \geq 2$.

To show (A.7), we first observe that, by increasing the constant $C$, we can assume $N$ to be sufficiently large. Then we expand (A.1) into

$$
m(z)+\frac{1}{m(z)+z}=-\frac{1}{N} \sum_{k=1}^{N} \frac{1}{m(z)+z} \delta_{k}(z) \frac{1}{m(z)+z-\delta_{k}(z)} .
$$

We define the complex random variable

$$
Y(z)=\frac{1}{N} \sum_{k=1}^{N} \frac{1}{m(z)+z} \delta_{k}(z) \frac{1}{m(z)+z-\delta_{k}(z)} .
$$

From (A.3) and since, by Theorem 4.2 of [15],

$$
\mathbb{P}\left(\sup _{1 \leq k \leq N}\left|\delta_{k}(z)\right| \geq \delta\right) \leq C e^{-c \min \left(\delta \sqrt{N y}, \delta^{2} N y\right)}
$$

for all $y \geq(\log N)^{4} / N$ and $\delta>0$, we find

$$
\mathbb{P}(|Y(z)| \geq \delta) \leq \mathbb{P}(|m(z)+z| \leq c)+\mathbb{P}\left(\sup _{k \leq N}\left|\delta_{k}(z)\right| \geq \min \left(\frac{c^{2} \delta}{2}, \frac{c}{2}\right)\right) \leq C e^{-c \delta \sqrt{N y}}
$$

for $\delta$ small enough, $y \geq(\log N)^{4} / N$, and $N$ large enough (independently of $\delta$ ).

To prove (A.7) for $|x|<2$, we use that, from (6.14) in [15],

$$
\left|m+\frac{1}{m+z}\right| \leq \delta \Rightarrow\left|m-m_{s c}\right| \leq \frac{C \delta}{(2-|x|)^{1 / 2}}
$$

for all $z=x+i y$ with $|x|<2$ and $0<y<1$. This implies, using (A.10), that

$$
\mathbb{P}\left(\left|m(z)-m_{s c}(z)\right| \geq \delta\right) \leq \mathbb{P}\left(|Y(z)| \geq c \delta(2-|x|)^{1 / 2}\right) \leq C e^{-c \delta \sqrt{N y(2-|x|)}}
$$


for all $\delta$ small enough, $N$ large enough, $|x| \leq 2,(\log N)^{4} / N \leq y \leq 1$.

It remains to show A.7) for $2 \leq|x| \leq K$. To this end, for $(\log N)^{4} / N \leq y \leq 1$ and $2 \leq|x| \leq K$, we consider the event

$$
\Omega^{*}=\left\{|m(z)+z| \geq c, \sup _{1 \leq k \leq N}\left|\delta_{k}(z)\right| \leq \delta,|Y(z)| \leq \delta \sqrt{|2-| x||}\right\}, \quad z=x+i y .
$$

From (A.3), A.9) and A.10), we have $\mathbb{P}\left(\left[\Omega^{*}\right]^{c}\right) \leq e^{-c \delta \sqrt{N y|2-| x||}}$ for all $\delta$ small and $N$ large enough. Solving (A.8) for $m$ on the set $\Omega^{*}$, we get

$$
m(z)=-\frac{z}{2}-\frac{Y(z)}{2}+\sqrt{\frac{z^{2}}{4}-1-\frac{z Y(z)}{2}+\frac{Y(z)^{2}}{4}} .
$$

Since $m(z)$ is the Stieltjes transform of an empirical measure with finite support, it is analytic away from a compact subset of the real axis. Similarly, on the set $\Omega^{*}, Y(z)$ is bounded and analytic away from a compact subset of the real axis. The square root in the above formula is therefore uniquely defined as the branch analytic on $\mathbb{C} \backslash(-\infty, 0]$, characterized by the property that the real part of the square root is non-negative. Hence, on $\Omega^{*}$,

$$
\begin{aligned}
m(z)-m_{s c}(z) & =-\frac{Y(z)}{2}+\sqrt{\frac{z^{2}}{4}-1-\frac{z Y(z)}{2}+\frac{Y(z)^{2}}{4}}-\sqrt{\frac{z^{2}}{4}-1} \\
& =-\frac{Y(z)}{2}-\frac{1}{4} \frac{2 z Y(z)-Y(z)^{2}}{\sqrt{\frac{z^{2}}{4}-1-\frac{z Y(z)}{2}+\frac{Y(z)^{2}}{4}}+\sqrt{\frac{z^{2}}{4}-1}}
\end{aligned}
$$

using the explicit formula (2.7) for $m_{s c}(z)$, and therefore

$$
\left|m(z)-m_{s c}(z)\right| \leq \frac{|Y(z)|}{2}+\frac{2|z||Y(z)|+|Y(z)|^{2}}{4 \operatorname{Re} \sqrt{\frac{z^{2}}{4}-1}} \leq C \frac{|Y(z)|+|Y(z)|^{2}}{\sqrt{|2-| x||}}
$$

using the fact that

$$
\operatorname{Re} \sqrt{\frac{z^{2}}{4}-1} \geq C|2-| x|| \quad \text { for all } 2 \leq|x| \leq K,|y| \leq 1 .
$$

From (A.11), we obtain that

$$
\mathbb{P}\left(\left|m(z)-m_{s c}(z)\right| \geq \delta\right) \leq \mathbb{P}\left(|Y(z)|+|Y(z)|^{2} \geq c \delta \sqrt{|2-| x||}\right) \leq e^{-c \delta \sqrt{N y|2-| x||}}
$$

for all $\delta$ small enough, $2 \leq|x| \leq K,(\log N)^{4} / N \leq y \leq 1$, and $N$ large enough.

Step 3. Fluctuations of $m(z)$. Suppose that $|x| \leq K,(\log N)^{4} / N \leq y \leq 1$ and $N y|2-| x|| \geq(\log N)^{4}$. Then there exist constants $C, c>0$ such that

$$
\mathbb{P}(|m(z)-\mathbb{E} m(z)| \geq \delta) \leq C e^{-c \delta \sqrt{N y|2-| x||}}
$$

for all $0<\delta \leq \delta_{0}$, with $\delta_{0}$ small enough and all $N$ large enough. 
To show (A.12), we observe first that

$$
\left|\mathbb{E} m(z)-m_{s c}(z)\right| \leq \mathbb{E}\left|m(z)-m_{s c}(z)\right| \leq \int_{0}^{1 / y} \mathrm{~d} t \mathbb{P}\left(\left|m(z)-m_{s c}(z)\right| \geq t\right),
$$

where we used that $|m(z)| \leq y^{-1}$. Using (A.7), we obtain

$$
\left|\mathbb{E} m(z)-m_{s c}(z)\right| \leq \frac{C}{\sqrt{N y|2-| x||}}+\frac{1}{y} e^{-c \sqrt{N y|2-| x||}} \leq \frac{2 C}{\sqrt{N y|2-| x||}}
$$

for $N$ large enough. For $\delta \sqrt{N y|2-| x||} \geq 4 C$ we thus obtain

$$
\mathbb{P}(|m(z)-\mathbb{E} m(z)| \geq \delta) \leq \mathbb{P}\left(\left|m(z)-m_{s c}(z)\right| \geq \frac{\delta}{2}\right) \leq C e^{-c \delta \sqrt{N y|2-| x||}}
$$

where we used (A.7) again. For $\delta \sqrt{N y|2-| x||} \leq 4 C$ the bound (A.12) is trivial.

As a consequence of (A.12), we immediately obtain (4.7). If $N y|2-| x|| \leq(\log N)^{4}$, we directly use (4.4). Otherwise, we use

$$
\mathbb{E}|m(z)-\mathbb{E} m(z)|^{q} \leq C_{q} \int_{0}^{\delta_{0}} t^{p-1} \mathbb{P}(|m(z)-\mathbb{E} m(z)| \geq t) \mathrm{d} t+C y^{-q} e^{-c \delta_{0} \sqrt{N y|2-| x||}}
$$

from (A.12), and we obtain the first term on the r.h.s. of (4.7).

Step 4. Convergence to the semicircle in expectation. Assume that $|x| \leq K,(\log N)^{4} / N \leq y \leq 1$ and $N y|2-| x|| \geq(\log N)^{4}$. Then

$$
\left|\mathbb{E} m(z)-m_{s c}(z)\right| \leq \frac{C}{N y|2-| x||^{3 / 2}}
$$

for a universal constant $C$. Note that this bound gains an additional $(N \eta)^{-1 / 2}$ factor on the precision of the estimates compared with Step 2 and Step 3, but the negative power of $|2-| x||$ has increased.

To prove (A.14), with $c_{0}:=\inf _{z}\left|m_{s c}(z)+z\right|>0$, we have

$$
|\mathbb{E} m(z)+z| \geq\left|m_{s c}(z)+z\right|-\left|\mathbb{E} m(z)-m_{s c}(z)\right| \geq c_{0}-\frac{C}{\sqrt{N y|2-| x||}} \geq \frac{c_{0}}{2}
$$

for $N$ large enough (here we used (A.13)). Expanding the denominator in the r.h.s. of (A.1) around $\mathbb{E} m(z)+z$, we find

$$
\begin{aligned}
m(z)= & -\frac{1}{\mathbb{E} m(z)+z}-\frac{1}{N} \sum_{k=1}^{N} \frac{1}{(\mathbb{E} m(z)+z)^{2}}\left(m(z)-\mathbb{E} m(z)+\delta_{k}(z)\right) \\
& +\frac{1}{N} \sum_{k=1}^{N} \frac{1}{(\mathbb{E} m(z)+z)^{2}}\left(m(z)-\mathbb{E} m(z)+\delta_{k}(z)\right)^{2} \frac{1}{m(z)+z-\delta_{k}(z)} .
\end{aligned}
$$


Taking expectation, we find

$$
\begin{aligned}
\mathbb{E} m(z)+\frac{1}{\mathbb{E} m(z)+z}= & -\frac{1}{(\mathbb{E} m(z)+z)^{2}} \mathbb{E} \delta_{1}(z) \\
& +\frac{1}{(\mathbb{E} m(z)+z)^{2}} \mathbb{E}\left[\left(m(z)-\mathbb{E} m(z)+\delta_{1}(z)\right)^{2} \frac{1}{m(z)+z-\delta_{1}(z)}\right] .
\end{aligned}
$$

With a Schwarz inequality, we get

$$
\begin{aligned}
\left|\mathbb{E} m(z)+\frac{1}{\mathbb{E} m(z)+z}\right| \leq & \frac{1}{|\mathbb{E} m(z)+z|^{2}}\left|\mathbb{E} \delta_{1}(z)\right| \\
& +2 \frac{\left(\mathbb{E}|m(z)-\mathbb{E} m(z)|^{4}+\mathbb{E}\left|\delta_{1}(z)\right|^{4}\right)^{1 / 2}}{|\mathbb{E} m(z)+z|^{2}}\left(\mathbb{E} \frac{1}{\left|m(z)+z-\delta_{1}(z)\right|^{2}}\right)^{1 / 2} .
\end{aligned}
$$

From (A.12), we find

$$
\mathbb{E}|m(z)-\mathbb{E} m(z)|^{q} \leq \frac{C_{q}}{(N y|2-| x||)^{q / 2}}
$$

for arbitrary $q \geq 1$. Moreover, with $c$ fixed in (A.3), we have

$$
\mathbb{P}\left(\left|m(z)+z-\delta_{1}(z)\right| \leq \frac{c}{2}\right) \leq \mathbb{P}(|m(z)+z| \leq c)+\mathbb{P}\left(\left|\delta_{1}(z)\right| \geq \frac{c}{2}\right) \leq e^{-C \sqrt{N y}} \leq e^{-C(\log N)^{2}}
$$

using (A.9), and hence

$$
\mathbb{E} \frac{1}{\left|m(z)+z-\delta_{1}(z)\right|^{q}} \leq \frac{1}{y^{q}} e^{-C(\log N)^{2}}+\frac{2^{q}}{c^{q}} \leq \frac{2^{q+1}}{c^{q}}
$$

if $N$ is large enough. Here we used the fact that $\operatorname{Im} m(z)+z-\delta_{1}(z) \geq \operatorname{Im} z=y$. From (A.9), we also have

$$
\mathbb{E}\left|\delta_{1}(z)\right|^{q} \leq \frac{C^{q}}{(N y)^{q / 2}} .
$$

From the definition of $\delta_{1}(z)$ in A.2, from $\mathbb{E} X^{(k)}=0$ and from A.5), we get

$$
\left|\mathbb{E} \delta_{1}(z)\right| \leq \frac{1}{N y}
$$

Combining this bound with (A.19), we find, from (A.18), that

$$
\left|\mathbb{E} m(z)+\frac{1}{\mathbb{E} m(z)+z}\right| \leq \frac{C}{N y|2-| x||} .
$$

Recall that $m_{s c}(z)$ solves the equation

$$
m_{s c}(z)+\frac{1}{m_{s c}(z)+z}=0 .
$$

This equation is stable in a sense that the inverse of the function $m \rightarrow m+(m+z)^{-1}$ near zero is Lipschitz continuous with a constant proportional to $|2-| x||^{1 / 2}$. Thus we obtain

$$
\left|\mathbb{E} m(z)-m_{s c}(z)\right| \leq \frac{C}{\left.N y|2-| x\right|^{3 / 2}}
$$


and this completes Step 4.

Step 5. Alternative bound on $\left|\mathbb{E} m(z)-m_{s c}(z)\right|$. Assuming $|x| \leq K$ and $(\log N)^{4} / N \leq y \leq 1$, there exists a constant $C>0$ such that

$$
\left|\mathbb{E} m(z)-m_{s c}(z)\right| \leq \frac{C}{N y^{3 / 2}|2-| x||^{1 / 2}}
$$

for all $N$ large enough (independently of $z=x+i y$ ).

To prove (A.24), we use the bound

$$
\mathbb{E}|m(z)-\mathbb{E} m(z)|^{q} \leq \frac{C^{q}}{\left(N y^{3 / 2}\right)^{q}}
$$

which is valid for all $q \geq 1$ and it follows from Theorem 3.1 in [13]. Expanding again the denominator in the r.h.s. of (A.1) around $\mathbb{E} m(z)+z$, we get

$$
\begin{aligned}
m(z)= & -\frac{1}{\mathbb{E} m(z)+z}-\frac{1}{N} \sum_{k=1}^{N} \frac{m(z)-\mathbb{E} m(z)+\delta_{k}(z)}{[\mathbb{E} m(z)+z]\left[m(z)+z-\delta_{k}(z)\right]} \\
= & -\frac{1}{\mathbb{E} m(z)+z}-\frac{1}{N} \sum_{k=1}^{N} \frac{m(z)-\mathbb{E} m(z)}{[\mathbb{E} m(z)+z]\left[m(z)+z-\delta_{k}(z)\right]}-\frac{1}{N} \sum_{k=1}^{N} \frac{\delta_{k}(z)}{(\mathbb{E} m(z)+z)^{2}} \\
& -\frac{1}{N} \sum_{k=1}^{N} \frac{\delta_{k}(z)\left(\mathbb{E} m(z)-m(z)+\delta_{k}(z)\right)}{[\mathbb{E} m(z)+z]^{2}\left[m(z)+z-\delta_{k}(z)\right]} .
\end{aligned}
$$

Taking the expectation, we find

$$
\begin{aligned}
\mid \mathbb{E} m(z)+ & \frac{1}{\mathbb{E} m(z)+z} \mid \\
\leq & \frac{1}{|\mathbb{E} m(z)+z|}\left(\mathbb{E}|m(z)-\mathbb{E} m(z)|^{2}\right)^{1 / 2}\left(\mathbb{E} \frac{1}{\left|m(z)+z-\delta_{1}(z)\right|^{2}}\right)^{1 / 2} \\
& +\frac{\left|\mathbb{E} \delta_{1}(z)\right|}{|\mathbb{E} m(z)+z|^{2}}+\frac{\left(\mathbb{E}\left|\delta_{1}(z)\right|^{4}\right)^{1 / 2}}{|\mathbb{E} m(z)+z|^{2}}\left(\mathbb{E} \frac{1}{\left|m(z)+z-\delta_{1}(z)\right|^{2}}\right)^{1 / 2} \\
& +\frac{\left(\mathbb{E}\left|\delta_{1}(z)\right|^{4}\right)^{1 / 4}\left(\mathbb{E}|m(z)-\mathbb{E} m(z)|^{4}\right)^{1 / 4}}{|\mathbb{E} m(z)+z|^{2}}\left(\mathbb{E} \frac{1}{\left|m(z)+z-\delta_{1}(z)\right|^{2}}\right)^{1 / 2} .
\end{aligned}
$$

Using A.20 with $q=2$, A.25) with $q=2$ and $q=4$, (A.21) with $q=4$ and (A.22), we find, by the stability argument, that

$$
\left|\mathbb{E} m(z)+\frac{1}{\mathbb{E} m(z)+z}\right| \leq \frac{C}{N y^{3 / 2}}
$$

which implies (A.24). This completes the proof of Theorem 4.1. 


\section{B Proof of Proposition 4.2}

We start with the proof of (4.14). From the moment method, we know that if $\lambda_{\min }(H)$ and $\lambda_{\max }(H)$ denote the smallest and the largest eigenvalues of the hermitian Wigner matrix $H$, and if $K$ is large enough, then

$$
\mathbb{P}\left(\lambda_{\min }(H) \leq-K\right)=\mathbb{P}\left(\lambda_{\max }(H) \geq K\right) \leq K^{-c N^{2 / 3}}
$$

(for example, one can use the bound $\mathbb{E} \operatorname{Tr} H^{N^{2 / 3}} \leq C$ from [28]; the symmetry condition on the distribution can be removed by symmetrization). This implies that $\mathfrak{N}(E) \leq N K^{-c N^{2 / 3}}$ for $E<-K$ and $1-\mathfrak{N}(E) \leq N K^{-c N^{2 / 3}}$ for all $E>K$. Therefore

$$
\int_{-\infty}^{\infty}\left|\mathfrak{N}(E)-\mathfrak{N}_{s c}(E)\right| \mathrm{d} E \leq \int_{-K}^{K}\left|[\mathfrak{N}(E)-\mathfrak{N}(-K)]-\mathfrak{N}_{s c}(E)\right| \mathrm{d} E+2 N K^{-c N^{2 / 3}}
$$

for $K>0$ large enough. The last term is negligible. The main estimate is contained in the following lemma whose proof is given at the end of this section.

Lemma B.1. Let $\varrho^{*}=\varrho_{+}-\varrho_{-}$be a difference of two finite measures with support in $[-K, K]$ for some $K>0$. Let

$$
m^{*}(z)=\int_{\mathbb{R}} \frac{\varrho^{*}(\mathrm{~d} x)}{x-z}, \quad \mathfrak{N}^{*}(E):=\int_{-K}^{E} \varrho^{*}(\mathrm{~d} x)
$$

be the Stieltjes transform and the distribution function of $\varrho^{*}$, respectively. Denote moreover by $m_{ \pm}^{*}(z)$ the Stieltjes transforms of $\varrho_{ \pm}^{*}$. We assume that $m^{*}, m_{+}^{*}, m_{-}^{*}$ satisfy the following bounds for $|x| \leq K+1$ :

$$
\begin{array}{lrl}
\left|m_{+}^{*}(x+i y)\right|+\left|m_{-}^{*}(x+i y)\right| \leq L_{1} & \text { for all } & (\log N)^{4} / N \leq|y| \leq 1 \\
\left|m^{*}(x+i y)\right| \leq \frac{L_{2}}{\left.N|y|^{3 / 2}|2-| x\right|^{1 / 2}} & \text { for all } & (\log N)^{4} / N \leq|y| \leq 1 \\
\left|m^{*}(x+i y)\right| \leq \frac{L_{3}}{N|y||2-| x||^{3 / 2}} & \text { for all } \quad N|y||2-| x|| \geq(\log N)^{4},
\end{array}
$$

with some constants $L_{1}, L_{2}, L_{3}$. Then

$$
\int_{-K}^{K}\left|\mathfrak{N}^{*}(E)\right| \mathrm{d} E \leq \frac{C L}{N^{6 / 7}}
$$

with $L=\max \left\{L_{1}, L_{2}, L_{3}\right\}$. The constant $C$ in (B.5) depends only on $K$.

We apply this lemma for the signed measure $\varrho^{*}(\mathrm{~d} x)=\mathbf{1}(|x| \leq K)\left[\varrho(x)-\varrho_{s c}(x)\right] \mathrm{d} x$. The bounds (B.2), (B.3), and (B.4) follow from (4.4), (4.8) and (4.9) (choosing $K+1$ instead of $K$ ), respectively. From (B.5) we obtain

$$
\int_{-K}^{K}\left|[\mathfrak{N}(E)-\mathfrak{N}(-K)]-\mathfrak{N}_{s c}(E)\right| \mathrm{d} E \leq \frac{C}{N^{6 / 7}},
$$

which, together with (B.1), completes the proof of (4.14). 
For the proof of (4.13), we fix $|E| \leq K$ and we choose $N^{-3 / 4} \leq \eta \leq 1$ to be optimized later. Define a function $f=f_{E}: \mathbb{R} \rightarrow \mathbb{R}$ such that $f(x)=1$ for $x \leq E-\eta, f(x)=0$ for $x>E+\eta$ with $\left|f^{\prime}\right| \leq C \eta^{-1}$ and $\left|f^{\prime \prime}\right| \leq C \eta^{-2}$. We have

$$
\left|\left(\frac{\mathscr{N}(-\infty, E)}{N}-\mathfrak{N}(E)\right)-\int_{-\infty}^{\infty} f_{E}(\lambda)[\omega(\mathrm{d} \lambda)-\varrho(\lambda) \mathrm{d} \lambda]\right| \leq \frac{\mathscr{N}[E-\eta, E+\eta]}{N}+\mathbb{E} \frac{\mathscr{N}[E-\eta, E+\eta]}{N}
$$

The second term on the r.h.s is estimated by $C \eta$, using (4.5). For the first term we use Theorem 4.6 of [15]:

$$
\mathbb{P}\left(\frac{\mathscr{N}[E-\eta, E+\eta]}{N} \geq \delta / 4\right) \leq C e^{-c \sqrt{\delta N}}
$$

with some positive $c>0$.

Now we consider the fluctuation of the smoothed distribution function

$$
W:=\int_{-\infty}^{\infty} f_{E}(\lambda)[\omega(\mathrm{d} \lambda)-\varrho(\lambda) \mathrm{d} \lambda]=\frac{1}{N} \sum_{\alpha=1}^{N}\left[f_{E}\left(\lambda_{\alpha}\right)-\mathbb{E} f_{E}\left(\lambda_{\alpha}\right)\right]
$$

We partition $[-K-2, K+2]$ into intervals $I_{r}$ of length $\eta$. For $M \geq M_{0}$ with a sufficiently large $M_{0}$, and set

$$
\Omega_{k}:=\left\{k M N \eta \leq \sup _{r} \mathcal{N}\left(I_{r}\right)<(k+1) M N \eta\right\}, \quad k=0,1,2, \ldots, \eta^{-1},
$$

then from Theorem 4.6 of [15] we know that

$$
\mathbb{P}\left\{\Omega_{k}\right\} \leq C e^{-c \sqrt{k M N \eta}} .
$$

Analogously to the calculation (D.16), the size of the variance of $W$ is determined by the size of $|\nabla W|$. On the event $\Omega_{k}$, we have

$$
\begin{aligned}
|\nabla W|^{2} & =\frac{1}{N} \sum_{1 \leq i \leq j \leq N}\left|\frac{\partial W}{\partial \operatorname{Re} h_{i j}}\right|^{2}+\left|\frac{\partial W}{\partial \operatorname{Im} h_{i j}}\right|^{2} \\
& =\frac{1}{N} \sum_{1 \leq i \leq j \leq N}\left|\frac{1}{N} \sum_{\alpha} f_{E}^{\prime}\left(\lambda_{\alpha}\right) \operatorname{Re} \overline{\mathbf{u}}_{\alpha}(i) \cdot \mathbf{u}_{\alpha}(j)\right|^{2}+\left|\frac{1}{N} \sum_{\alpha} f_{E}^{\prime}\left(\lambda_{\alpha}\right) \operatorname{Re} \overline{\mathbf{u}}_{\alpha}(i) \cdot \mathbf{u}_{\alpha}(j)\right|^{2} \\
& =\frac{1}{N^{3}} \sum_{\alpha}\left|f^{\prime}\left(\lambda_{\alpha}\right)\right|^{2} \leq \frac{k M}{N^{2} \eta}
\end{aligned}
$$

(Note that the derivative in $\nabla W$ is with respect to the original random variables $z_{i j}=\sqrt{N} h_{i j}$ ). From the concentration inequality (Theorem 2.1 of [4]) we obtain that

$$
\begin{aligned}
\mathbb{P}(W \geq \delta / 4) & \leq e^{-T \delta / 4} \mathbb{E} e^{T W} \\
& \leq e^{-T \delta / 4} \mathbb{E} \exp \left[S T^{2}|\nabla W|^{2}\right] \\
& \leq C e^{-T \delta / 4} \sum_{k=0}^{1 / \eta} \mathbb{E} \mathbf{1}_{\Omega_{k}} e^{S T^{2} k M N^{-2} \eta^{-1}} \\
& \leq C e^{-T \delta / 4} \sum_{k=0}^{1 / \eta} e^{-c \sqrt{k M N \eta}} e^{S T^{2} k M N^{-2} \eta^{-1}} .
\end{aligned}
$$


Choosing $T=c N^{1 / 2}$, and $\eta=N^{-3 / 4}$, it follows that

$$
\mathbb{P}(W \geq \delta / 4) \leq e^{-\delta \sqrt{N}} .
$$

Repeating the same argument with $W$ replaced by $-W$, we conclude that

$$
\mathbb{P}(|W| \geq \delta / 4) \leq e^{-c \delta \sqrt{N}}
$$

Combining this with (B.6) and (B.7), we have

$$
\mathbb{P}\left(\left|\mathfrak{N}(E)-\mathfrak{N}_{s c}(E)\right| \geq \delta\right) \leq C e^{-c \delta \sqrt{N}} .
$$

which completes the proof of Proposition 4.2 .

Proof of Lemma B.1. For simplicity, in the proof we omit the star from the notation. First notice that (B.2) implies that, after taking imaginary part,

$$
|\varrho|(I) \leq C L_{1}|I|
$$

for any interval of length $|I| \geq(\log N)^{4} / N, I \subset[-K-1, K+1]$.

Let $(\log N)^{4} / N \leq \eta \leq 1$ to be chosen later. Fix $E \in[-K, K]$ and define a function $f=f_{E}: \mathbb{R} \rightarrow \mathbb{R}$ such that $f(x)=1$ for $x \in[-K, E-\eta], f(x)=0$ for $x>E+\eta$ and $x<-K-1$ with $\left|f^{\prime}\right| \leq C \eta^{-1}$ and $\left|f^{\prime \prime}\right| \leq C \eta^{-2}$. We have

$$
\left|\mathfrak{N}(E)-\int_{-\infty}^{\infty} f_{E}(\lambda) \varrho(\mathrm{d} \lambda)\right| \leq|\varrho|(E-\eta, E+\eta) \leq C L_{1} \eta .
$$

To express $f(\lambda)$ in terms of the Stieltjes transform, we use the Helffer-Sjöstrand functional calculus, see, e.g., [8]. Let $\chi(y)$ be a smooth cutoff function with support in $[-1,1]$, with $\chi(y)=1$ for $|y| \leq 1 / 2$ and with bounded derivatives. Let

$$
\tilde{f}(x+i y)=\left(f(x)+i y f^{\prime}(x)\right) \chi(y)
$$

then

$$
f(\lambda)=\frac{1}{2 \pi} \int_{\mathbb{R}^{2}} \frac{\partial_{\bar{z}} \tilde{f}(x+i y)}{\lambda-x-i y} \mathrm{~d} x \mathrm{~d} y=\frac{1}{2 \pi} \int_{\mathbb{R}^{2}} \frac{i y f^{\prime \prime}(x) \chi(y)+i\left(f(x)+i y f^{\prime}(x)\right) \chi^{\prime}(y)}{\lambda-x-i y} \mathrm{~d} x \mathrm{~d} y,
$$

and therefore, since $f$ is real,

$$
\begin{aligned}
\left|\int_{-\infty}^{\infty} f(\lambda) \varrho(\mathrm{d} \lambda)\right|= & \operatorname{Re} \int_{-\infty}^{\infty} f(\lambda) \varrho(\mathrm{d} \lambda) \mid \\
\leq & \left|\frac{1}{2 \pi} \int_{\mathbb{R}^{2}} y f^{\prime \prime}(x) \chi(y) \operatorname{Im} m(x+i y) \mathrm{d} x \mathrm{~d} y\right| \\
& +C \int_{\mathbb{R}^{2}}\left(|f(x)|+|y|\left|f^{\prime}(x)\right|\right)\left|\chi^{\prime}(y)\right||m(x+i y)| \mathrm{d} x \mathrm{~d} y .
\end{aligned}
$$


Using (B.3) and the support properties of $\chi^{\prime}$ and $f$, the second contribution is bounded by

$$
\begin{aligned}
L_{2} \int_{|x| \leq K+1} \mathrm{~d} x \int_{\frac{1}{2} \leq|y| \leq 1} \mathrm{~d} y \frac{\left[|f(x)|+|y|\left|f^{\prime}(x)\right|\right]}{N|y|^{3 / 2}|2-| x||^{1 / 2}} & \leq \frac{C L_{2}}{N}+\frac{C L_{2}}{N \eta} \int_{E-\eta}^{E+\eta} \frac{\mathrm{d} x}{\left.|2-| x\right|^{1 / 2}} \\
& \leq \frac{C L_{2}}{\left.N|2-| E\right|^{1 / 2}} .
\end{aligned}
$$

For the first term in (B.13), we split the integration:

$$
\begin{aligned}
& \left|\int_{\mathbb{R}^{2}} y f^{\prime \prime}(x) \chi(y) \operatorname{Im} m(x+i y) \mathrm{d} x \mathrm{~d} y\right| \\
& \leq C \int_{|y| \leq \eta} \int_{|x| \leq K+1}|y|\left|f^{\prime \prime}(x)\right|\left(\left|\operatorname{Im} m_{+}(x+i y)\right|+\left|\operatorname{Im} m_{-}(x+i y)\right|\right) \mathrm{d} x \mathrm{~d} y \\
& \quad+\left|\int_{\eta \leq|y| \leq 1} \int_{|x| \leq K+1} y f^{\prime \prime}(x) \chi(y) m(x+i y) \mathrm{d} x \mathrm{~d} y\right|
\end{aligned}
$$

where, in the second term, we dropped the imaginary part since $f$ and $\chi$ are real. To bound the first term we note that, for every fixed $x$, the functions

$$
|y|\left|\operatorname{Im} m_{ \pm}(x+i y)\right|=\int \rho_{ \pm}(\mathrm{d} s) \frac{y^{2}}{(s-x)^{2}+y^{2}}
$$

are monotonically increasing in $|y|$. This implies that, for all $|y| \leq \eta$,

$$
|y|\left|\operatorname{Im} m_{ \pm}(x+i y)\right| \leq \eta\left|\operatorname{Im} m_{ \pm}(x+i \eta)\right| \leq C \eta
$$

by (B.2). Therefore, we find

$$
\int_{|y| \leq \eta} \int_{|x| \leq K+1}|y|\left|f^{\prime \prime}(x)\right|\left(\left|\operatorname{Im} m_{+}(x+i y)\right|+\left|\operatorname{Im} m_{-}(x+i y)\right|\right) \mathrm{d} x \mathrm{~d} y \leq C L_{1} \eta .
$$

As for the second term on the r.h.s. of (B.15), we integrate by parts first in $x$, then in $y$. It is sufficient to consider the regime $\eta \leq y \leq 1$, the case of negative $y$ 's is treated identically. We find

$$
\begin{aligned}
& \int_{\eta \leq y \leq 1} \int_{|x| \leq K+1} i y f^{\prime \prime}(x) \chi(y) m(x+i y) \mathrm{d} y \mathrm{~d} x \\
&=-\int_{\eta \leq y \leq 1} \int_{|x| \leq K+1} i y f^{\prime}(x) \chi(y) m^{\prime}(x+i y) \mathrm{d} y \mathrm{~d} x \\
&= \int_{\eta \leq y \leq 1} \int_{|x| \leq K+1} \partial_{y}(y \chi(y)) f^{\prime}(x) m(x+i y) \mathrm{d} y \mathrm{~d} x \\
&+\int_{|x| \leq K+1} \eta f^{\prime}(x) \chi(\eta) m(x+i \eta) \mathrm{d} x .
\end{aligned}
$$

Using (B.2), the second term is bounded in absolute value by

$$
C L_{1} \int_{|x| \leq K+1} \eta\left|f^{\prime}(x)\right| \mathrm{d} x \leq C L_{1} \eta .
$$


The absolute value of the first term on the r.h.s. of (B.17) is estimated by

$$
C \eta^{-1} \int_{\eta}^{1} \int_{E-\eta}^{E+\eta}|m(x+i y)| \mathrm{d} x \mathrm{~d} y
$$

Putting all terms together, we find from (B.11), (B.13), (B.14), (B.16) and (B.18) that

$$
\int_{|E| \leq K}|\mathfrak{N}(E)| \mathrm{d} E \leq C L\left(\eta+N^{-1}+\int_{\eta}^{1} \mathrm{~d} y \int_{|x| \leq K+1} \mathrm{~d} x|m(x+i y)|\right) .
$$

We will use the bounds $(B .2)-(B .4)$ and we split the integration into separate regions:

$$
\begin{aligned}
\int_{\eta}^{1} \mathrm{~d} y & \int_{|x| \leq K+1} \mathrm{~d} x|m(x+i y)| \\
\leq & C L \int_{\eta}^{1} \mathrm{~d} y \int \mathrm{d} x \mathbf{1}\left(|2-| x|| \leq \frac{(\log N)^{4}}{N y}\right) \\
& +C L \int_{\mathrm{d} y} \mathrm{~d} \int_{|x| \leq K+1} \mathrm{~d} x \min \left(1, \frac{1}{N|y|^{3 / 2}|2-| x||^{1 / 2}}, \frac{1}{N|y||2-| x||^{3 / 2}}\right) \\
= & : C L(\mathrm{I}+\mathrm{II}) .
\end{aligned}
$$

Clearly

$$
\mathrm{I} \leq \int_{\eta}^{1} \mathrm{~d} y \int \mathrm{d} x \mathbf{1}\left(|2-| x|| \leq \frac{(\log N)^{4}}{N y}\right) \leq C \frac{(\log N)^{5}}{N} .
$$

As for the second term on the r.h.s. of (B.21), we divide the integral into several pieces:

$$
\begin{aligned}
\mathrm{II} \leq & \int_{\eta}^{N^{-4 / 7}} \mathrm{~d} y\left[\int_{|2-| x|| \leq(N y)^{-2 / 3}} \mathrm{~d} x+\int_{(N y)^{-2 / 3} \leq|2-| x|| \leq K+3} \mathrm{~d} x \frac{1}{N y|2-| x||^{3 / 2}}\right] \\
& +\int_{N^{-4 / 7}}^{1} \mathrm{~d} y\left[\int_{|2-| x|| \leq y^{1 / 2}} \mathrm{~d} x \frac{1}{N y^{3 / 2}|2-| x||^{1 / 2}}+\int_{y^{1 / 2} \leq|2-| x|| \leq K+3} \mathrm{~d} x \frac{1}{\left.N y|2-| x\right|^{3 / 2}}\right] \\
\leq & \int_{\eta}^{N^{-4 / 7}} \mathrm{~d} y \frac{1}{(N y)^{2 / 3}}+\int_{N^{-4 / 7}}^{1} \mathrm{~d} y \frac{1}{N y^{5 / 4}} \\
\leq & C N^{-6 / 7}
\end{aligned}
$$

independently of $\eta$. Inserting in (B.20), and choosing $\eta=N^{-6 / 7}$, we conclude the proof of B.5).

\section{Proof of Lemma 4.3.}

We partition the interval $[-2+\kappa, 2-\kappa]$ into a disjoint union of intervals

$$
I_{r}=\left[n^{\gamma} N^{-1}\left(r-\frac{1}{2}\right), n^{\gamma} N^{-1}\left(r+\frac{1}{2}\right)\right]
$$


of length $n^{\gamma} N^{-1}$ and center $w_{r}=r n^{\gamma} N^{-1}$, where $r \in \mathbb{Z},|r| \leq r_{1}:=N n^{-\gamma}(2-\kappa)$. Then, for any $r$,

$$
\left|\frac{\mathscr{N}\left(I_{r}\right)}{n^{\gamma}}-\varrho_{s c}\left(w_{r}\right)\right| \leq n^{-\gamma / 6}
$$

by (4.16). To prove (4.21), first we locate middle eigenvalue. Let $r_{0}$ be the index such that

$$
\sum_{r<r_{0}} \mathscr{N}\left(I_{r}\right)<\frac{N}{2} \leq \sum_{r \leq r_{0}} \mathscr{N}\left(I_{r}\right)
$$

in other words

$$
\lambda_{N / 2} \in I_{r_{0}}
$$

For definiteness, we can assume that $r_{0} \geq 0$. Using the second event in (4.18) we obtain that

$$
\sum_{r=1}^{r_{0}-1} \mathscr{N}\left(I_{r}\right) \leq N / 2-\mathscr{N}[(-\infty, 0)] \leq C N n^{-\gamma / 6}
$$

On the other hand, with the notation $r_{1}:=\min \left\{\left(r_{0}-1\right)_{+}, N n^{-\gamma}\right\}$, we have by (C.2) that

$$
\sum_{r=1}^{r_{0}-1} \mathscr{N}\left(I_{r}\right) \geq \sum_{r=1}^{r_{1}} \mathscr{N}\left(I_{r}\right) \geq n^{\gamma}\left(1-n^{-\gamma / 6}\right) \sum_{r=1}^{r_{1}} \varrho_{s c}\left(w_{r}\right) \geq c r_{1} n^{\gamma}
$$

where we used that $w_{r} \leq 1$ for any $r \leq r_{1} \leq N n^{-\gamma}$ and thus $\varrho_{s c}\left(w_{r}\right) \geq \varrho_{s c}(1) \geq c$. From (C.4) and (C.5) we conclude that $r_{0} \leq C N n^{-7 \gamma / 6}$, i.e. $w_{r_{0}} \leq C n^{-\gamma / 6}$. Thus we proved that

$$
\left|w_{r_{0}}\right| \leq C n^{-\gamma / 6}, \quad\left|\lambda_{N / 2}\right| \leq C n^{-\gamma / 6} \text {. }
$$

Starting the proof of (4.21), we can assume that $a \geq N / 2$ by symmetry. Suppose first that $\lambda_{a} \in$ $[-2+\kappa, 2-\kappa]$, i.e. $\lambda_{a} \in I_{r}$ for some $|r| \leq r_{1}$, i.e. $a \geq N / 2$ implies $r \geq r_{0}$. Then we have

$$
\sum_{u=r_{0}+1}^{r-1} \mathscr{N}\left(I_{u}\right) \leq a-N / 2 \leq \sum_{u=r_{0}}^{r} \mathscr{N}\left(I_{u}\right)
$$

i.e.

$$
\sum_{u=r_{0}+1}^{r-1} \mathscr{N}\left(I_{u}\right) \leq a-N \int_{-\infty}^{0} \varrho_{s c}(E) \mathrm{d} E \leq \sum_{u=r_{0}}^{r} \mathscr{N}\left(I_{u}\right)
$$

using $\int_{-\infty}^{0} \varrho_{s c}(E) \mathrm{d} E=1 / 2$.

Note that

$$
\sum_{u=r_{0}}^{r} \mathscr{N}\left(I_{u}\right) \leq n^{\gamma}\left(1+C n^{-\gamma / 6}\right) \sum_{u=r_{0}}^{r} \varrho_{s c}\left(w_{u}\right) \leq\left(1+C n^{-\gamma / 6}\right) N \int_{0}^{w_{r}} \varrho_{s c}(E) \mathrm{d} E
$$

using (C.2) and that $\gamma$ is small. Similarly

$$
\sum_{u=r_{0}+1}^{r-1} \mathscr{N}\left(I_{u}\right) \geq\left(1-C n^{-\gamma / 6}\right) N \int_{0}^{w_{r}} \varrho_{s c}(E) \mathrm{d} E-C n^{\gamma} .
$$


Thus, combining these estimates with (C.7), we have

$$
\left|a N^{-1}-\int_{-\infty}^{w_{r}} \varrho_{s c}(E) \mathrm{d} E\right| \leq C n^{-\gamma / 6}
$$

i.e.

$$
\left|\mathfrak{N}_{s c}^{-1}\left(a N^{-1}\right)-w_{r}\right| \leq C \kappa^{-1 / 2} n^{-\gamma / 6}
$$

using (11.10) and $\kappa^{3 / 2} \leq a N^{-1} \leq 1-\kappa^{3 / 2}$. Since $\lambda_{a} \in I_{r}$, i.e. $\left|\lambda_{a}-w_{r}\right| \leq n^{\gamma} N^{-1}$, we obtain (4.21). Finally, we consider the case when $\lambda_{a}>2-\kappa$. The lower bound in (C.7) and the estimate (C.9) hold with $r=r_{1}$ so we get

$$
\begin{aligned}
a \geq & \left(1-C n^{-\gamma / 6}\right) N \int_{-\infty}^{w_{r_{1}}} \varrho_{s c}(E) \mathrm{d} E-C n^{\gamma} \\
& \geq N \int_{-\infty}^{2-\kappa} \varrho_{s c}(E) \mathrm{d} E-C n^{-\gamma / 6} N \geq\left(1-\pi^{-1} \kappa^{3 / 2}\right) N-C n^{-\gamma / 6} N,
\end{aligned}
$$

which contradicts the assumption $a \leq N\left(1-\kappa^{3 / 2}\right)$ for large $N$.

For the proof of (4.22), suppose that $\lambda_{a} \in I_{r}, \lambda_{b} \in I_{s}$. Using (4.21) and $N \kappa^{3 / 2} \leq a<b \leq N\left(1-\kappa^{3 / 2}\right)$, we know that $-2+\kappa / 2 \leq w_{r} \leq w_{s} \leq 2-\kappa / 2$. By (4.21), we have the apriori bound

$$
\left|\lambda_{a}-\lambda_{b}\right| \leq\left|\mathfrak{N}_{s c}^{-1}\left(a N^{-1}\right)-\mathfrak{N}_{s c}^{-1}\left(b N^{-1}\right)\right|+C_{\kappa} n^{-\gamma / 6} \leq C_{\kappa} N^{-1}|b-a|+C_{\kappa} n^{-\gamma / 6} \leq C_{\kappa} n^{-\gamma / 6}
$$

by the assumption $|b-a| \leq C N n^{\gamma / 6}$. In particular

$$
\left|w_{r}-w_{s}\right| \leq\left|\lambda_{a}-\lambda_{b}\right|+C_{\kappa} n^{-\gamma / 6} \leq C_{\kappa} n^{-\gamma / 6} .
$$

The constants $C_{\kappa}$ depend on $\kappa$ as $C_{\kappa} \leq C \kappa^{1 / 2}$.

From $\lambda_{a} \in I_{r}, \lambda_{b} \in I_{s}$ it also follows that

$$
(s-r-1) n^{\gamma} N^{-1} \leq \lambda_{b}-\lambda_{a} \leq(s-r+1) n^{\gamma} N^{-1}
$$

and

$$
\sum_{u=r+1}^{s-1} \mathscr{N}\left(I_{u}\right) \leq b-a \leq \sum_{u=r}^{s} \mathscr{N}\left(I_{u}\right) .
$$

Let $s-r+1=\sum_{j=0}^{j_{0}} 2^{m_{j}}, m_{0}<m_{1}<\ldots$ be the binary representation of $s-r+1$ with $j_{0}=$ $\left[\log _{2}(s-r+1)\right] \leq \log N$. Using this representation, we can concatanate the intervals $I_{u}, r \leq u \leq s$, into longer intervals $J_{0}, J_{1}, \ldots$ of length $\left|J_{j}\right|=2^{j} n^{\gamma} N^{-1}$ such that

$$
I:=\bigcup_{u=r}^{s} I_{r}=\bigcup_{j=0}^{j_{0}} J_{j}
$$

Since $\varrho_{s c}^{\prime}$ is bounded on $I$, we have

$$
\varrho_{s c}(w) \leq\left(1+C_{\kappa}|I|\right) \varrho_{s c}\left(\lambda_{a}\right), \quad \text { for any } w \in I .
$$


On the set $\Omega$ we thus have (see (4.16))

$$
\begin{aligned}
\sum_{u=r}^{s} \mathscr{N}\left(I_{u}\right)=\sum_{j=0}^{j_{0}} \mathscr{N}\left(J_{j}\right) & \leq\left(1+C_{\kappa}|I|\right) \varrho_{s c}\left(\lambda_{a}\right) \sum_{j=0}^{j_{0}} N\left|J_{j}\right|\left[1+\left(N\left|J_{j}\right|\right)^{-1 / 4} n^{\gamma / 12}\right] \\
& \leq\left(1+C_{\kappa}|I|\right) \varrho_{s c}\left(\lambda_{a}\right)\left[N|I|+(N|I|)^{3 / 4} n^{\gamma / 12} \log N\right] .
\end{aligned}
$$

Similary, one can get a lower bound on $\sum_{u=r+1}^{s-1} \mathscr{N}\left(I_{u}\right)$. Recalling $|I|=(s-r+1) n^{\gamma} N^{-1}$, and that $|I| \leq C n^{-\gamma / 6}$ from (C.11), we conclude from (C.13) that

$$
\left|(b-a)-\varrho_{s c}\left(\lambda_{a}\right) n^{\gamma}(s-r)\right| \leq C_{\kappa} n^{\gamma}|b-a|^{3 / 4}+C_{\kappa} N^{-1}|b-a|^{2}+C_{\kappa} n^{\gamma} .
$$

But from (C.12)

$$
\left|N \varrho_{s c}\left(\lambda_{a}\right)\left(\lambda_{b}-\lambda_{a}\right)-\varrho_{s c}\left(\lambda_{a}\right) n^{\gamma}(s-r)\right| \leq C_{\kappa} n^{\gamma}
$$

thus

$$
\left|N \varrho_{s c}\left(\lambda_{a}\right)\left(\lambda_{b}-\lambda_{a}\right)-(b-a)\right| \leq C_{\kappa} n^{\gamma}|b-a|^{3 / 4}+C_{\kappa} N^{-1}|b-a|^{2}
$$

with $C_{\kappa} \leq C \kappa^{1 / 2}$, and we have proved (4.22).

\section{Proof of Lemma 5.3}

We start with the outline of the proof and indicate the origin of the restriction $\alpha>1 / 4$. We will first regularize the logarithmic interaction on a scale $\eta$ at the expense of an error of $O(\eta)$ for each pair of eigenvalues, modulo logarithmic corrections (Lemma D.1). By a Schwarz inequality (D.18), the fluctuation of the regularized two body interaction is split into the product of the fluctuation of the regularized potential $A_{x}(\mathrm{D} .14)$ and the fluctuation of the local semicircle law regularized on scale $\eta$. The latter is of order $O\left(N^{-1 / 2} \eta^{-1 / 2}\right)$ by the improved fluctuation bound on the local semicircle law (4.7). The former is of order $O\left(N^{-1} \eta^{-1 / 2}\right)$ using that the logarithmic Sobolev inequality (2.4) on the single site distribution can be turned into a spectral gap estimate for $A_{x}$. Finally, we optimize the regularization error $O(\eta)$ and the fluctuation error $O\left(N^{-3 / 2} \eta^{-1}\right)$ per particle pairs, which gives a total error of order $N^{2} \cdot N^{-3 / 4}=N^{1+1 / 4}$.

The proof of the following regularization lemma is postponed until the end of the section:

Lemma D.1. Let $(\log N)^{4} / N \leq \eta \leq 1$, then

$$
\left|\frac{1}{N^{2}} \mathbb{E}\left[\sum_{j<k} \log \left|\lambda_{j}-\lambda_{k}\right|-\frac{1}{N^{2}} \sum_{j<k} \log \left|\lambda_{j}-\lambda_{k}+i \eta\right|\right]\right| \leq C \eta \log N
$$

with respect to any Wigner ensemble whose single-site distribution satisfies (2.6) and (2.5).

Then Lemma 5.3 directly follows from the following statement:

Lemma D.2. Suppose $\eta=N^{-3 / 4}$, then

$$
\left|\frac{1}{N^{2}} \mathbb{E} \sum_{i<j} \log \right| \lambda_{i}-\lambda_{j}+i \eta\left|-\frac{1}{2} \iint \log \right| x-y\left|\varrho_{s c}(x) \varrho_{s c}(y) \mathrm{d} x \mathrm{~d} y\right| \leq C \frac{\log N}{N^{3 / 4}}
$$

for a universal constant $C>0$ and all $N$ large enough. 
Proof of Lemma D.2. Recall that $\omega(\mathrm{d} x)$ denotes the empirical measure of the eigenvalues (4.1). We have

$$
\left|\sum_{i<j} \log \right| \lambda_{i}-\lambda_{j}+i \eta\left|-\frac{N^{2}}{2} \int \log \right| x-y+i \eta|\omega(\mathrm{d} x) \omega(\mathrm{d} y)| \leq N|\log \eta|
$$

because of the contribution of the diagonal terms.

Step 1. Recall the definition of $\omega_{\eta}(x)$ from (4.2), then

$$
\left|\mathbb{E} \int \log \right| x-y+i \eta\left|\omega(\mathrm{d} x) \omega(\mathrm{d} y)-\mathbb{E} \int \mathrm{d} x \mathrm{~d} y \log \right| x-y+i \eta\left|\omega_{\eta}(x) \omega_{\eta}(y)\right| \leq C \eta(\log N)^{2} .
$$

To prove (D.3), we observe that

$$
\begin{aligned}
& \int \omega(\mathrm{d} x) \omega(\mathrm{d} y) \log |x-y+i \eta|-\int \mathrm{d} x \mathrm{~d} y \log |x-y+i \eta| \omega_{\eta}(x) \omega_{\eta}(y) \\
&=\int \omega(\mathrm{d} x) \omega(\mathrm{d} y) \int \mathrm{d} t \mathrm{~d} r \frac{\eta}{(t-x)^{2}+\eta^{2}} \frac{\eta}{(r-y)^{2}+\eta^{2}}(\log |x-y+i \eta|-\log |t-r+i \eta|) .
\end{aligned}
$$

Clearly

$$
\begin{aligned}
& \left|\mathbb{E} \int \omega(\mathrm{d} x) \omega(\mathrm{d} y) \log \right| x-y+i \eta\left|-\mathbb{E} \int \mathrm{d} x \mathrm{~d} y \log \right| x-y+i \eta\left|\omega_{\eta}(x) \omega_{\eta}(y)\right| \\
& \leq \mathbb{E} \int \omega(\mathrm{d} x) \omega(\mathrm{d} y) \int \mathrm{d} t \mathrm{~d} r \frac{\eta \mathbf{1}(|t-x| \leq 1)}{(t-x)^{2}+\eta^{2}} \frac{\eta \mathbf{1}(|r-y| \leq 1)}{(r-y)^{2}+\eta^{2}}|\log | x-y+i \eta|-\log | t-r+i \eta|| \\
& \quad+C \eta|\log \eta| .
\end{aligned}
$$

Here we also used that $\mathbb{P}\{\operatorname{supp} \omega \in[-K, K]\} \geq 1-e^{-C N}$ for some large constant $K$. Next we observe that

$$
\begin{aligned}
\int \mathrm{d} t & \mathrm{~d} r \frac{\eta \mathbf{1}(|t-x| \leq 1)}{(t-x)^{2}+\eta^{2}} \frac{\eta \mathbf{1}(|r-y| \leq 1)}{(r-y)^{2}+\eta^{2}}|\log | x-y+i \eta|-\log | t-r+i \eta|| \\
& \leq \int_{0}^{1} \mathrm{~d} s \int \mathrm{d} t \mathrm{~d} r \frac{\eta \mathbf{1}(|t-x| \leq 1)}{(t-x)^{2}+\eta^{2}} \frac{\eta \mathbf{1}(|r-y| \leq 1)}{(r-y)^{2}+\eta^{2}} \frac{|(x-y)-(t-r)|}{|s(t-r)+(1-s)(x-y)+i \eta|} \\
& \leq \eta \int_{0}^{1} \mathrm{~d} s \int \mathrm{d} t \mathrm{~d} r(|t|+|r|) \frac{\mathbf{1}\left(|t| \leq \eta^{-1}\right)}{t^{2}+1} \frac{\mathbf{1}\left(|r| \leq \eta^{-1}\right)}{r^{2}+1} \frac{1}{|s \eta(t-r)+(x-y)+i \eta|}
\end{aligned}
$$

Inserting this bound back into (D.4), we find

$$
\begin{aligned}
& \left|\mathbb{E} \int \omega(\mathrm{d} x) \omega(\mathrm{d} y) \log \right| x-y+i \eta\left|-\mathbb{E} \int \mathrm{d} x \mathrm{~d} y \log \right| x-y+i \eta\left|\omega_{\eta}(x) \omega_{\eta}(y)\right| \\
& \quad \leq C \eta|\log \eta|+C \eta \int_{0}^{1} \mathrm{~d} s \int \mathrm{d} t \mathrm{~d} r(|t|+|r|) \frac{\mathbf{1}\left(|t|,|r| \leq \eta^{-1}\right)}{\left(t^{2}+1\right)\left(r^{2}+1\right)} \mathbb{E} \frac{1}{N^{2}} \sum_{i, j} \frac{1}{\left|\lambda_{i}-\lambda_{j}+s \eta(t-r)+i \eta\right|} \\
& \quad \leq C \eta(\log N)^{2} .
\end{aligned}
$$


Here we used the bound

$$
\frac{1}{N^{2}} \mathbb{E} \sum_{i, j} \frac{1}{\left|\lambda_{i}-\lambda_{j}+x \eta+i \eta\right|} \leq C \log N
$$

which holds uniformly in $x \in \mathbb{R}$, if $\eta \geq(\log N)^{4} / N$. To prove (D.7), consider the event

$$
\Theta_{0}=\left\{\max _{j}\left|\lambda_{j}\right| \leq K_{0}\right\}
$$

for some $K_{0}>0$. Moreover, define the intervals $I_{k}=[-(k+1) \eta,-k \eta] \cup[k \eta,(k+1) \eta]$, for all nonnegative integer $k \leq K_{0} / \eta$, and consider the event

$$
\Theta_{1}=\left\{\mathscr{N}_{I_{k}} \leq K N \eta, k=0,1,2, \ldots, K_{0} \eta^{-1}\right\}
$$

For sufficiently large $K_{0}$ and $K$ we have

$$
\mathbb{P}\left(\Theta_{0}^{c}\right) \leq e^{-c N K_{0}^{2}}, \quad \mathbb{P}\left(\Theta_{1}^{c}\right) \leq e^{-c \sqrt{K N \eta}}
$$

by Lemma 7.4 [13] and by 4.20), after adjusting $c$. Then

$$
\begin{aligned}
\frac{1}{N^{2}} \mathbb{E} \sum_{j<\ell} \frac{1}{\left|\lambda_{\ell}-\lambda_{j}+x \eta+i \eta\right|} & \leq \eta^{-1}\left[\mathbb{P}\left(\Theta_{0}^{c}\right)+\mathbb{P}\left(\Theta_{1}^{c}\right)\right]+\mathbb{E}\left[\frac{C}{N^{2}} \sum_{k, m}^{K_{0} \eta^{-1}} \frac{\mathscr{N}_{I_{k}} \mathscr{N}_{I_{m}} \mathbf{1}_{\Theta_{0} \cap \Theta_{1}}}{(|k-m+x|+1) \eta}\right] \\
& \leq \eta^{-1}\left(e^{-c K_{0}^{2} N}+e^{-c \sqrt{K N \eta}}\right)+C K^{2} \eta \sum_{k, m}^{K_{0} \eta^{-1}} \frac{1}{(|k-m+x|+1)} \\
& \leq C|\log \eta|
\end{aligned}
$$

because $N \eta \geq(\log N)^{4}$ by assumption. This completes the proof of Step 1 .

Step 2. Let $\varrho_{\eta}(x)=\mathbb{E} \omega_{\eta}(x)$, and assume $(N \eta) \geq(\log N)^{8}$, then

$\left|\mathbb{E} \int \log \right| x-y+i \eta\left|\omega_{\eta}(x) \omega_{\eta}(y) \mathrm{d} x \mathrm{~d} y-\int \log \right| x-y+i \eta\left|\varrho_{\eta}(x) \varrho_{\eta}(y) \mathrm{d} x \mathrm{~d} y\right| \leq C\left(\frac{1}{N^{3 / 2} \eta}+\frac{\eta^{1 / 2}}{N}\right)$

We note that

$$
\begin{aligned}
\mathbb{E} \int \log |x-y+i \eta| \omega_{\eta}(x) & \omega_{\eta}(y) \mathrm{d} x \mathrm{~d} y-\int \log |x-y+i \eta| \varrho_{\eta}(x) \varrho_{\eta}(y) \mathrm{d} x \mathrm{~d} y \\
& =\mathbb{E} \int \log |x-y+i \eta|\left(\omega_{\eta}(x)-\varrho_{\eta}(x)\right)\left(\omega_{\eta}(y)-\varrho_{\eta}(y)\right) \mathrm{d} x \mathrm{~d} y \\
& =\mathbb{E} \int \mathrm{d} x\left(A_{x}-\mathbb{E} A_{x}\right)\left(\omega_{\eta}(x)-\varrho_{\eta}(x)\right),
\end{aligned}
$$

where we defined the random variable

$$
A_{x}:=\int \mathrm{d} y \log |x-y+i \eta| \omega_{\eta}(y)=\frac{1}{N} \sum_{j} f_{\eta}\left(\lambda_{j}-x\right)
$$


with $f_{\eta}(\lambda)=\left(\log |\cdot| * \theta_{\eta}\right)(\lambda)$.

To estimate the fluctuations of $A_{x}$ we use that the logarithmic Sobolev inequality (2.4) implies the spectral gap, i.e., we have

$$
\mathbb{E}\left|A_{x}-\mathbb{E} A_{x}\right|^{2} \leq S \mathbb{E}\left|\nabla A_{x}\right|^{2} .
$$

Let $\mathbf{u}_{\alpha}$ denote the orthonormal set of eigenvectors belonging to the eigenvalues $\lambda_{\alpha}$ of $H$. Taking into account the scaling (2.1), we have

$$
\begin{aligned}
\left|\nabla A_{x}\right|^{2} & =\frac{1}{N} \sum_{1 \leq i \leq j \leq N}\left[\left|\frac{\partial A_{x}}{\partial \operatorname{Re} h_{i j}}\right|^{2}+\left|\frac{\partial A_{x}}{\partial \operatorname{Im} h_{i j}}\right|^{2}\right] \\
& =\frac{1}{N} \sum_{i j}\left[\left|\frac{1}{N} \sum_{\alpha} f_{\eta}^{\prime}\left(\lambda_{\alpha}-x\right) \operatorname{Re} \overline{\mathbf{u}}_{\alpha}(i) \mathbf{u}_{\alpha}(j)\right|^{2}+\left|\frac{1}{N} \sum_{\alpha} f_{\eta}^{\prime}\left(\lambda_{\alpha}-x\right) \operatorname{Im} \overline{\mathbf{u}}_{\alpha}(i) \mathbf{u}_{\alpha}(j)\right|^{2}\right] \\
& =\frac{1}{N^{3}} \sum_{\alpha, \beta} f_{\eta}^{\prime}\left(\lambda_{\alpha}-x\right) f_{\eta}^{\prime}\left(\lambda_{\beta}-x\right)\left|\mathbf{u}_{\alpha} \cdot \mathbf{u}_{\beta}\right|^{2} \\
& =\frac{1}{N^{3}} \sum_{\alpha}\left|f_{\eta}^{\prime}\left(\lambda_{\alpha}-x\right)\right|^{2} \\
& \leq \frac{C}{N^{2} \eta} \omega_{\eta}(x)
\end{aligned}
$$

using that $\left|f_{\eta}^{\prime}(\lambda)\right|^{2} \leq C\left(\lambda^{2}+\eta^{2}\right)^{-1}$. We have from (D.15), (D.16) and (4.5) that

$$
\mathbb{E}\left|A_{x}-\mathbb{E} A_{x}\right|^{2} \leq \frac{C}{N^{2} \eta}
$$

On the other hand, from (4.7) and $\omega_{\eta}(x)=\pi^{-1} \operatorname{Im} m(x+i \eta)$ we have

$$
\mathbb{E}\left|\omega_{\eta}(x)-\varrho_{\eta}(x)\right|^{q} \leq \frac{C_{q}}{(N \eta|2-| x||)^{q / 2}}+C_{q} \mathbf{1}\left(N \eta|2-| x|| \leq(\log N)^{4}\right)
$$

for all $q \geq 1$ and for $|x| \leq K$ with some large constant $K$.

In order to insert this estimate into (D.13), we need to extract the necessary decay for large $x$ from $\omega_{\eta}(x)-\varrho_{\eta}(x)$. For $|x| \geq 2 K_{0}$ sufficiently large and for any $q \geq 1$ we can estimate

$$
\begin{aligned}
\mathbb{E} \omega_{\eta}^{q}(x) & \leq \eta^{-q} \mathbb{E} \mathbf{1}\left(\left|\lambda_{\max }\right| \geq|x| / 2\right)+\mathbb{E} \mathbf{1}\left(\left|\lambda_{\max }\right| \leq|x| / 2\right)\left(\frac{1}{N} \sum_{\alpha} \frac{\eta}{\left(\lambda_{\alpha}-x\right)^{2}+\eta^{2}}\right)^{q} \\
& \leq \eta^{-q} e^{-c|x|^{2} N}+\frac{\eta^{q}}{|x|^{2 q}} \leq \frac{C_{q} \eta^{q}}{|x|^{2 q}} .
\end{aligned}
$$


Inserting the last three equations into (D.13) with $q=2$, we find

$$
\begin{aligned}
\left|\mathbb{E} \int \log \right| x-y+i \eta \mid & \omega_{\eta}(x) \omega_{\eta}(y) \mathrm{d} x \mathrm{~d} y-\int \log |x-y+i \eta| \varrho_{\eta}(x) \varrho_{\eta}(y) \mathrm{d} x \mathrm{~d} y \mid \\
& \leq \int \mathrm{d} x\left(\mathbb{E}\left|A_{x}-\mathbb{E} A_{x}\right|^{2}\right)^{1 / 2}\left(\mathbb{E}\left|\omega_{\eta}(x)-\varrho_{\eta}(x)\right|^{2}\right)^{1 / 2} \\
& \leq \frac{C K_{0}}{N^{3 / 2} \eta}+\frac{C(\log N)^{4}}{N^{2} \eta^{3 / 2}}+\frac{C}{N \eta^{1 / 2}} \int_{|x| \geq 2 K_{0}} \mathrm{~d} x \sqrt{\mathbb{E} \omega_{\eta}^{2}(x)} \\
& \leq \frac{C}{N^{3 / 2} \eta}+\frac{C \eta}{N \eta^{1 / 2}} \int_{|x| \geq 2 K_{0}} \frac{\mathrm{d} x}{|x|^{2}} .
\end{aligned}
$$

This completes the proof of Step 2 .

Step 3. We have

$$
\left|\int \mathrm{d} x \mathrm{~d} y \log \right| x-y+i \eta\left|\varrho_{\eta}(x) \varrho_{\eta}(y)-\int \mathrm{d} x \mathrm{~d} y \log \right| x-y+i \eta\left|\varrho_{s c}(x) \varrho_{s c}(y)\right| \leq C N^{-6 / 7}+C \eta \text {. }
$$

To prove (D.19), we write

$$
\begin{aligned}
\int \mathrm{d} x \mathrm{~d} y \log |x-y+i \eta| \varrho_{\eta}(x) \varrho_{\eta}(y) & -\int \mathrm{d} x \mathrm{~d} y \log |x-y+i \eta| \varrho_{s c}(x) \varrho_{s c}(y) \\
= & \int \mathrm{d} x \mathrm{~d} y \log |x-y+i \eta|\left(\varrho_{\eta}(x)-\varrho_{s c}(x)\right) \varrho_{\eta}(y) \\
& +\int \mathrm{d} x \mathrm{~d} y \log |x-y+i \eta| \varrho_{s c}(x)\left(\varrho_{\eta}(y)-\varrho_{s c}(y)\right) .
\end{aligned}
$$

To control the first term on the r.h.s. of the last equation, we recall that

$$
\mathfrak{N}(x)=\frac{1}{N} \mathbb{E} \mathscr{N}(-\infty ; x), \quad \mathfrak{N}_{s c}(x)=\int_{-\infty}^{x} \varrho_{s c}(t) \mathrm{d} t
$$

denote the expected number of eigenvalues up to $x$ normalized by $N$ (integrated density of states) and the distribution function of the semicircle law. Note that $\mathfrak{N}(x)-\mathfrak{N}_{s c}(x)$ vanishes at $x= \pm \infty$. Introducing $\mathfrak{N}_{\eta}(x):=\int_{-\infty}^{x} \varrho_{\eta}$ and integrating by parts we find

$$
\begin{aligned}
\left|\int \mathrm{d} x \mathrm{~d} y \log \right| x-y+ & i \eta\left|\left(\varrho_{\eta}(x)-\varrho_{s c}(x)\right) \varrho(y)\right| \\
= & \left|\int \mathrm{d} x \mathrm{~d} y \log \right| x-y+i \eta\left|\frac{\mathrm{d}}{\mathrm{d} x}\left(\mathfrak{N}_{\eta}(x)-\mathfrak{N}_{s c}(x)\right) \varrho(y)\right| \\
& =\left|\int \mathrm{d} x \mathrm{~d} y \frac{(x-y)}{(x-y)^{2}+\eta^{2}}\left(\mathfrak{N}_{\eta}(x)-\mathfrak{N}_{s c}(x)\right) \varrho(y)\right| \\
& =\left|\int \mathrm{d} x \operatorname{Re} \mathbb{E} m(x+i \eta)\left(\mathfrak{N}_{\eta}(x)-\mathfrak{N}_{s c}(x)\right)\right| \\
& \leq \sup _{x} \mathbb{E}|m(x+i \eta)| \int \mathrm{d} x\left|\mathfrak{N}_{\eta}(x)-\mathfrak{N}_{s c}(x)\right| .
\end{aligned}
$$


From the upper bound (4.4) on $|\mathbb{E} m(x+i \eta)|$ and from

$$
\int \mathrm{d} x\left|\mathfrak{N}_{s c}(x)-\left(\mathfrak{N}_{s c} * \theta_{\eta}\right)(x)\right| \leq C \eta
$$

we find, by (4.14),

$$
\begin{aligned}
\left|\int \mathrm{d} x \mathrm{~d} y \log \right| x-y+i \eta\left|\left(\varrho_{\eta}(x)-\varrho_{s c}(x)\right) \varrho(y)\right| & \leq C \eta+C \int \mathrm{d} x\left|\mathfrak{N}_{\eta}(x)-\left(\mathfrak{N}_{s c} * \theta_{\eta}\right)(x)\right| \\
& \leq C \eta+C \int \mathrm{d} x\left|\mathfrak{N}(x)-\mathfrak{N}_{s c}(x)\right| \\
& \leq C \eta+C N^{-6 / 7} .
\end{aligned}
$$

The second term on the r.h.s. of (D.20) can be bounded similarly. This completes the proof of Step 3. Combining the estimates in Step 1-3 and choosing $\eta=N^{-3 / 4}$, we finish the proof of the Lemma D.2,

Proof of Lemma D.1. We split the summation into three parts:

$$
\sum_{j<k} \log \left|\lambda_{j}-\lambda_{k}+i \delta\right|=Y_{1}(\delta)+Y_{2}(\delta)+Y_{3}(\delta)
$$

for any $0 \leq \delta \leq \eta$ with

$$
\begin{aligned}
& Y_{1}(\delta)=\sum_{j<k} 1\left(\left|\lambda_{j}-\lambda_{k}\right| \geq \eta\right) \log \left|\lambda_{j}-\lambda_{k}+i \delta\right| \\
& Y_{2}(\delta)=\sum_{j<k} 1\left(N^{-10} \leq\left|\lambda_{j}-\lambda_{k}\right| \leq \eta\right) \log \left|\lambda_{j}-\lambda_{k}+i \delta\right| \\
& Y_{3}(\delta)=\sum_{j<k} 1\left(\left|\lambda_{j}-\lambda_{k}\right| \leq N^{-10}\right) \log \left|\lambda_{j}-\lambda_{k}+i \delta\right| .
\end{aligned}
$$

We have

$$
\begin{aligned}
\mathbb{E}\left|Y_{1}(\eta)-Y_{1}(0)\right| & \leq \mathbb{E} \sum_{j<k} \mathbf{1}\left(\left|\lambda_{j}-\lambda_{k}\right| \geq \eta\right) \int_{0}^{1} \mathrm{~d} s\left|\frac{\mathrm{d}}{\mathrm{d} s} \log \right| \lambda_{j}-\lambda_{k}+i s \eta|| \\
& \leq \mathbb{E} \sum_{j<k} \mathbf{1}\left(\left|\lambda_{j}-\lambda_{k}\right| \geq \eta\right) \int_{0}^{1} \mathrm{~d} s \frac{\eta}{\left|\lambda_{j}-\lambda_{k}+i s \eta\right|} \\
& \leq \mathbb{E} C \eta \sum_{j<k} \frac{1}{\left|\lambda_{j}-\lambda_{k}+i \eta\right|} \\
& \leq C N^{2} \eta|\log N|
\end{aligned}
$$

by (D.7). For the $Y_{2}$ term, we remark that, for arbitrary $0 \leq \delta \leq \eta$,

$$
\left|Y_{2}(\delta)\right| \leq C \log N \sum_{j<k} \mathbf{1}\left(\left|\lambda_{j}-\lambda_{k}\right| \leq \eta\right)
$$


To bound the r.h.s. we consider the events $\Theta_{0}, \Theta_{1}$ from (D.8), (D.9) with sufficiently large $K$ and $K_{0}$ so that (D.10) holds. Then

$$
\begin{aligned}
\mathbb{E}\left|Y_{2}(\delta)\right| \leq & C N^{2}(\log N)\left(\mathbb{P}\left(\Theta_{0}^{c}\right)+\mathbb{P}\left(\Theta_{1}^{c}\right)\right) \\
& +C(\log N) \mathbb{E}\left[\mathbf{1}\left(\Theta_{0} \cap \Theta_{1}\right) \sum_{k=0}^{K_{0} \eta^{-1}} \mathscr{N}_{I_{k}}\left(\mathscr{N}_{I_{k-1}}+\mathscr{N}_{k}+\mathscr{N}_{I_{k+1}}\right)\right] \\
\leq & C N^{2}(\log N) e^{-c(\log N)^{2}}+C(\log N) \eta^{-1}(N \eta)^{2} \\
\leq & C N^{2}(\log N) \eta
\end{aligned}
$$

for every $0 \leq \delta \leq \eta$. Finally, for the $Y_{3}$ term we use the level repulsion estimate (E.5) from Theorem E.3. which implies that for any interval $I=[E-\varepsilon / N, E+\varepsilon / N]$ with $E \in \mathbb{R}$ and $0<\varepsilon \leq 1$

$$
\mathbb{P}\left(\mathscr{N}_{I} \geq 2\right) \leq C \varepsilon^{4} N^{18}
$$

Let

$$
J_{r}=\left[\frac{r-1}{N^{10}}, \frac{r+1}{N^{10}}\right], \quad r \in \mathbb{Z}
$$

be overlapping intervals covering $\mathbb{R}$. We can then write

$$
\left|Y_{3}(\delta)\right| \leq \sum_{j<k} \sum_{r \in \mathbb{Z}} \sum_{m=0}^{\infty} 1\left\{\lambda_{j} \in J_{r}, \frac{2^{-m-1}}{N^{10}} \leq\left|\lambda_{j}-\lambda_{k}\right| \leq \frac{2^{-m}}{N^{10}}\right\}\left|\log \left(2^{m} N^{10}\right)\right| .
$$

We split the interval $J_{r}$ into overlapping subintervals of length $2^{-m+1} N^{-10}$ by defining

$$
J_{r, s}:=\left[\frac{r-1}{N^{10}}+\frac{s}{2^{m} N^{10}}, \frac{r-1}{N^{10}}+\frac{s+2}{2^{m} N^{10}}\right], \quad 0 \leq s \leq 2^{m+1}-2 .
$$

Then

$$
\begin{aligned}
\mathbb{P}\left\{\lambda_{j} \in I_{r}, \frac{2^{-m-1}}{N^{10}} \leq\left|\lambda_{j}-\lambda_{k}\right| \leq \frac{2^{-m}}{N^{10}}\right\} & \leq \sum_{s=0}^{2^{m}} \mathbb{P}\left\{\lambda_{j} \in I_{r}, \mathscr{N}_{J_{r, s}} \geq 2\right\} \\
& \leq \frac{2^{m} C N^{18}}{\left(2^{m-1} N^{9}\right)^{4}} \leq C 2^{-3 m} N^{-18}
\end{aligned}
$$

For large $|r| \geq K N^{10}$, we can also use the bound

$$
\mathbb{P}\left\{\lambda_{j} \in I_{r}\right\} \leq C \exp \left(-c N\left(N^{-10} r\right)^{2}\right),
$$

that follows from the trivial large deviation estimate for the largest eigenvalue (Lemma 7.4 [13]). Inserting these last two estimates into (D.26), we have for every $0 \leq \delta \leq \eta$

$$
\begin{aligned}
\mathbb{E}\left|Y_{3}(\delta)\right| & \leq C N^{2} \sum_{m=0}^{\infty}(m+\log N)\left[\sum_{|r| \leq r^{*}} 2^{-3 m} N^{-18}+\sum_{|r|>r^{*}} \exp \left(-c N\left(N^{-10} r\right)^{2}\right)\right] \\
& \leq C N^{-2}(\log N),
\end{aligned}
$$

where $r^{*}=K N^{10} \log (m+2)$ for brevity. Combining (D.23), (D.25), and (D.28), we obtain (D.1). 


\section{E Level repulsion near the spectral edge}

We need to establish a Wegner-type inequality, and bounds on the level repulsion in the same spirit as in Theorem 3.4 and Theorem 3.5 of [15], for energy intervals close to the spectral edges. Since we only need these bounds for very small values of $\varepsilon \simeq N^{-\alpha}$, we are not aiming at the most general result here. The statements we present can be proven by simply replacing, in the proof of Theorems 3.4 and Theorem 3.5 of [15], the convergence to the semicircle law stated in Theorem 3.1 of [15] with Theorem 4.1. Recall that Theorem 3.1 of [15] is valid up to the smallest possible scale $\eta>K / N$ but only away from the spectral edges, while Theorem 4.1 holds all the way to the spectral edges, but only up to the logarithmic scale $\eta>(\log N)^{4} / N$. A better $N$-dependence of the bounds in the following theorem (but a worse $\kappa$-dependence) can be achieved by following the dependence on $\kappa$ of the constants in Theorem 3.1 of [15].

All statements assume the conditions (2.4)-(2.6). We introduce the notation that $[x]_{+}$denotes the positive part of a real number $x$.

Theorem E.1 (Gap distribution). Let $H$ be an $N \times N$ hermitian Wigner matrix and let $|E|<2$. Denote by $\lambda_{\alpha}$ the largest eigenvalue below $E$ and assume that $\alpha \leq N-1$. Then there are positive constants $C, D, c, d$ such that

$$
\mathbb{P}\left(\lambda_{\alpha+1}-E \geq \frac{K}{N}, \alpha \leq N-1\right) \leq C e^{-c[2-|E|]^{3 / 2} \sqrt{K}}
$$

for any $N \geq 1$ and any $D(\log N)^{4} /(2-|E|) \leq K \leq \kappa N d$.

Proof. The proof of this theorem can be obtained following the proof of Theorem 3.3 in [15], making use of Theorem 4.1 instead of Theorem 3.1 of [15] (in order to follow the $|2-| E||$ dependence of the probability). More precisely, we observe that the event $\lambda_{\alpha+1}-E \geq K / N$ implies that there is a gap of size $K / N$ about the energy $E^{\prime}=E+K /(2 N)$. Choosing $M=D^{1 / 2} \kappa^{-1 / 2}$ for a sufficiently large constant $D>0$, and $\eta=K /\left(N M^{2}\right) \geq(\log N)^{4}$, we find, similarly to (7.3)-(7.4) in [15], that, apart from a set $\Omega^{c}$ of measure $\mathbb{P}\left(\Omega^{c}\right) \leq C e^{-c \sqrt{K}}$,

$$
\operatorname{Im} m\left(E^{\prime}+i \eta\right) \leq \frac{16}{M} \leq \frac{16 \sqrt{\kappa}}{D}
$$

which implies, for sufficiently large $D$, that

$$
\left|m\left(E^{\prime}+i \eta\right)-m_{s c}\left(E^{\prime}+i \eta\right)\right| \geq \frac{c_{0}}{5}
$$

where $c_{0}=\pi \varrho\left(E^{\prime}\right) \geq c \sqrt{\kappa}$. The theorem then follows because, by Theorem 4.1, the event (E.2) has probability

$$
\mathbb{P}\left(\left|m\left(E^{\prime}+i \eta\right)-m_{s c}\left(E^{\prime}+i \eta\right)\right| \geq c \kappa^{1 / 2}\right) \leq C e^{-c \kappa^{1 / 2} \sqrt{N \eta \kappa}} \leq C e^{-c \kappa^{3 / 2} \sqrt{K}}
$$

Theorem E.2 (Wegner Estimate). Let $E \in \mathbb{R}$ and set $\kappa:=[2-|E|]_{+}$. There exists a constant $C>0$ such that for the number of eigenvalues $\mathscr{N}_{I}$ in the interval $I=[E-\varepsilon /(2 N) ; E+\varepsilon /(2 N)]$, we have

$$
\mathbb{E} \mathscr{N}_{I} \leq \frac{C \varepsilon(\log N)^{4}}{\left(\kappa+N^{-1}\right)^{9}}
$$


for every $E \in \mathbb{R}$ and $\varepsilon \leq 1$. Moreover

$$
y \mathbb{E}|m(x+i y)|^{2} \leq \frac{C(\log N)^{4}}{\left(\kappa+N^{-1}\right)^{9}}
$$

for all $x \in \mathbb{R}, y>0$.

Theorem E.3 (Level Repulsion). Let $E \in \mathbb{R}$ and set $\kappa:=[2-|E|]_{+}$. There exists a universal constant $C$ such that for the number of eigenvalues $\mathscr{N}_{I}$ in the interval $I=[E-\varepsilon /(2 N) ; E+\varepsilon /(2 N)]$ we have

$$
\mathbb{P}\left(\mathscr{N}_{I} \geq 2\right) \leq \frac{C \varepsilon^{4}(\log N)^{4}}{\left(\kappa+N^{-1}\right)^{18}}
$$

for all $E \in \mathbb{R}$, all $0<\varepsilon<1$, and all $N$ large enough.

Proof. The proof of Theorem E.2 and Theorem E.3 follows exactly the proof of Theorem 3.4 and, respectively, Theorem 3.5 in [15], after replacing Theorem 3.3 of [15] by Theorem E.1 above (in order to follow the dependence on the distance from the edges).

Note that the results of the last three theorems are only useful in the regime of very small $\varepsilon=N|I| \ll$ $(\log N)^{-4}$.

\section{F Properties of the equilibrium measure}

Here we check the conditions (a) and (b) in Theorem 11.1. The main ingredient is the following:

Lemma F.1. Let $L \in \mathscr{G}$ and $\mathbf{y} \in \mathscr{Y}_{L}$. After rescaling, then for any fixed $\sigma>0$ with $J^{\prime}=[-1+\sigma / 2,1-$ $\sigma / 2]$, the first and second derivatives of the potential are uniformly bounded on $J^{\prime}$, i.e.

$$
\sup _{x \in J^{\prime}}\left|U_{\widetilde{\mathbf{y}}}^{(\ell)}(x)\right| \leq C_{\ell}, \quad \ell=1,2,
$$

where the constant is independent of $\mathbf{y}$. Furthermore, the endpoints $a, b$ of the support of the equilibrium measure $v=v_{\mathbf{y}}$ satisfy

$$
|a+1|,|b-1| \leq C n^{-\gamma / 3} \log n .
$$

Condition (b) of Theorem 11.1 is given now by (F.1). To see condition (a) of Theorem 11.1, let $\left[a_{n}, b_{n}\right]$ denote the support of the equilibrium measure $v_{n}$, then $a_{n} \rightarrow-1$ and $b_{n} \rightarrow 1$ as $n \rightarrow \infty$, thus $g_{n}$ is positive on $J=[-1+\sigma, 1-\sigma]$ for any fixed $\sigma>0$ and any sufficiently large $n$.

For the uniform boundedness of $g_{n}(x)$ on $J$, we use the explicit formula (see, e.g. Theorem 2.5. of [24]):

$$
g_{n}(x)=\frac{1}{2 \pi^{2}} \sqrt{\left(x-a_{n}\right)\left(b_{n}-x\right)} \text { P.V. } \int_{a_{n}}^{b_{n}} \frac{V_{n}^{\prime}(s)}{s-x} \frac{1}{\sqrt{\left(s-a_{n}\right)\left(b_{n}-s\right)}} \mathrm{d} s,
$$

where P.V. denoted principal value. For sufficiently large $n$ and for any $x \in J$ the singularity of $(s-x)^{-1}$ is uniformly separated away from $a_{n}$ and $b_{n}$, i.e. from the singularity of the square roots. Moreover, $V_{n}^{\prime}(x)$ is a smooth function inside $(-1,1)$ with

$$
\sup _{n} \sup _{x \in J^{\prime}}\left|V_{n}^{\prime}(x)\right|+\left|V_{n}^{\prime \prime}(x)\right| \leq C .
$$


according to (F.1). Thus the uniform boundedness of $g_{n}$ on $J$ follows immediately from (F.3) with standard estimates on the principal value.

Proof of Lemma F.1. Recall the definition $E_{L}=\mathfrak{N}_{s c}^{-1}\left(L N^{-1}\right)$ from 6.11). For $\mathbf{y} \in \mathscr{Y}_{L}$ we know from the first bound in (6.13) that $\operatorname{dist}\left(I_{\mathrm{y}}, E_{L}\right) \leq C n^{-\gamma / 6}$, and from (4.22) that

$$
y_{k}=y_{-1}+\frac{k+O\left(k^{4 / 5}\right)}{N \varrho_{0}},
$$

with $\varrho_{0}:=\varrho_{s c}\left(E_{L}\right)$, assuming $\gamma \leq 1 / 20$ and $C n \leq|k| \leq n^{B} \leq N^{1 / 2}$. After rescaling, this corresponds to

$$
\tilde{y}_{k}=\frac{k+O\left(k^{4 / 5}\right)}{n \varrho_{0}}, \quad \varrho_{0}:=\varrho_{s c}\left(E_{L}\right),
$$

and we will drop the tilde for the rest of this proof. This bound on the location of $y_{k}$ 's will be used to estimate the derivatives of $U_{\mathbf{y}}$. For $\ell=1,2$ and $x \in J^{\prime}$ we have

$$
\begin{aligned}
\left|U_{\mathrm{y}}^{(\ell)}(x)\right| & \leq \frac{2}{n} \sum_{|k|<C n} \frac{1}{\left|x-y_{k}\right|^{\ell}}+\frac{2}{n}\left|\sum_{C n \leq|k|<n^{B}} \frac{1}{\left(x-y_{k}\right)^{\ell}}\right| \\
& \leq C \sigma^{-\ell}+\frac{C}{n} \sum_{C n \leq k<n^{B}}\left|\frac{1}{\left(x-y_{k}\right)^{\ell}}+\frac{1}{\left(x-y_{-k}\right)^{\ell}}\right| .
\end{aligned}
$$

For $\ell=2$ we can use in the second sum that $\left|x-y_{ \pm k}\right| \geq C k\left[n \varrho_{0}\right]^{-1}$ for $k \geq C n$ by (F.4), thus $\left|U_{\mathrm{y}}^{\prime \prime}(x)\right| \leq C(\sigma)$. For $\ell=1$ we estimate

$$
\left|\frac{1}{x-y_{k}}+\frac{1}{x-y_{-k}}\right|=\left|\frac{2 x-y_{k}-y_{-k}}{\left(x-y_{k}\right)\left(x-y_{-k}\right)}\right| \leq \frac{2|x| n \varrho_{0}+C k^{4 / 5}}{k^{2}} n \varrho_{0}
$$

where we used (F.4) and $k \geq C n$. After summation we conclude that $\left|U_{\mathbf{y}}^{\prime}(x)\right| \leq C(\sigma)$ and thus (F.1) is proven.

To estimate the location of the endpoints, we substitute $V(x)=U_{\mathbf{y}}(x)$ into the equations (11.2). We have

$$
\begin{aligned}
\frac{2}{n} \sum_{|k|<n^{B}} \int_{a}^{b} \frac{1}{\sqrt{(s-a)(b-s)}} \frac{\mathrm{d} s}{s-y_{k}} & =0 \\
\frac{1}{n \pi} \sum_{|k|<n^{B}} \int_{a}^{b} \frac{s}{\sqrt{(s-a)(b-s)}} \frac{\mathrm{d} s}{s-y_{k}} & =-1 .
\end{aligned}
$$

We will need the following explicit integration formulae for $a<b$ (see, e.g. Formula 2.266 in [17])

$$
\begin{aligned}
& \int_{a}^{b} \frac{1}{\sqrt{(s-a)(b-s)}} \frac{\mathrm{d} s}{s-y}=\frac{\pi}{\sqrt{(a-y)(b-y)}} \quad \text { if } \quad y<a<b, \\
& \int_{a}^{b} \frac{1}{\sqrt{(s-a)(b-s)}} \frac{\mathrm{d} s}{s-y}=-\frac{\pi}{\sqrt{(a-y)(b-y)}} \quad \text { if } \quad a<b<y .
\end{aligned}
$$




$$
\int_{a}^{b} \frac{\mathrm{d} s}{\sqrt{(s-a)(b-s)}}=\pi
$$

With these formulae, (F.6) and (F.7) can be written as

$$
\begin{gathered}
\frac{1}{n} \sum_{-n^{B}<k \leq-1} \frac{1}{\sqrt{\left(a-y_{k}\right)\left(b-y_{k}\right)}}-\frac{1}{n} \sum_{1 \leq k<n^{B}} \frac{1}{\sqrt{\left(a-y_{k}\right)\left(b-y_{k}\right)}}=0, \\
\frac{1}{n} \sum_{-n^{B}<k \leq-1}\left[\frac{y_{k}}{\sqrt{\left(a-y_{k}\right)\left(b-y_{k}\right)}}+1\right]-\frac{1}{n} \sum_{1 \leq k<n^{B}}\left[\frac{y_{k}}{\sqrt{\left(a-y_{k}\right)\left(b-y_{k}\right)}}-1\right]=-1 .
\end{gathered}
$$

Using the bound (F.4) on the location of $y_{k}$ 's, we replace the limit $-n^{B}<k$ with $-Y \leq y_{k}$ and the limit $k<n^{B}$ with $y_{k} \leq Y$ in the summations in (F.10) and (F.11), where $Y:=n^{B-1} \varrho_{0}^{-1}$. We have, for example, for the first sum (F.10),

$$
\frac{1}{n} \sum_{-n^{B}<k \leq-1} \frac{1}{\sqrt{\left(a-y_{k}\right)\left(b-y_{k}\right)}}=\frac{1}{n} \sum_{-Y<y_{k} \leq-1} \frac{1}{\sqrt{\left(a-y_{k}\right)\left(b-y_{k}\right)}}+O\left(n^{-1 / 5} Y^{-1}\right),
$$

and the estimate for the other three sums in (F.10), (F.11) is identical.

With similar argument, we can remove the $y_{k}$ 's that are too close to $[-1,1]$. Let $X=n^{\gamma-1}$, then

$$
\frac{C}{n \sqrt{a+1}} \leq \frac{1}{n} \sum_{-1-X<y_{k} \leq-1} \frac{1}{\sqrt{\left(a-y_{k}\right)\left(b-y_{k}\right)}} \leq \frac{C n^{\gamma-1}}{\sqrt{(a+1)(b+1)}},
$$

where, for the lower bound, we used that $y_{-1}=-1$, while for the upper bound we used that the number of $y_{k}$ 's in $[-1-X,-1]$ is at most $C n^{\gamma}$ (see the third set in the definition of (4.18)). Similarly we have

$$
\frac{C}{n \sqrt{1-b}} \leq \frac{1}{n} \sum_{1 \leq y_{k}<1+X} \frac{1}{\sqrt{\left(a-y_{k}\right)\left(b-y_{k}\right)}} \leq \frac{C n^{\gamma-1}}{\sqrt{(1-b)(1-a)}}
$$

and for the sums in F.11

$$
\begin{aligned}
& -\frac{C n^{\gamma-1}}{\sqrt{(a+1)(b+1)}} \leq \frac{1}{n} \sum_{-1-X<y_{k} \leq-1}\left[\frac{y_{k}}{\sqrt{\left(a-y_{k}\right)\left(b-y_{k}\right)}}+1\right] \leq C n^{\gamma-1}-\frac{C}{n \sqrt{a+1}} \\
& -C n^{\gamma-1}+\frac{C}{n \sqrt{1-b}} \leq \frac{1}{n} \sum_{1 \leq y_{k}<1+X}\left[\frac{y_{k}}{\sqrt{\left(a-y_{k}\right)\left(b-y_{k}\right)}}-1\right] \leq \frac{C n^{\gamma-1}}{\sqrt{(1-b)(1-a)}} .
\end{aligned}
$$

Define

$$
\begin{array}{r}
W_{1}:=\frac{1}{n} \sum_{-Y<y_{k}<-1-X} \frac{1}{\sqrt{\left(a-y_{k}\right)\left(b-y_{k}\right)}}-\frac{1}{n} \sum_{1+X<y_{k}<Y} \frac{1}{\sqrt{\left(a-y_{k}\right)\left(b-y_{k}\right)}} \\
W_{2}:=\frac{1}{n} \sum_{-Y<y_{k}<-1-X}\left[\frac{y_{k}}{\sqrt{\left(a-y_{k}\right)\left(b-y_{k}\right)}}+1\right]-\frac{1}{n} \sum_{1+X<y_{k}<Y}\left[\frac{y_{k}}{\sqrt{\left(a-y_{k}\right)\left(b-y_{k}\right)}}-1\right]+1
\end{array}
$$

to be the truncated summations. Combining the above estimates with estimates of type (F.12) and using $B \geq 2$ so that $n^{\gamma} Y^{-1} \leq n^{\gamma-1}$, we get from (F.10), (F.11) that

$$
\frac{C}{n \sqrt{1-b}}-\frac{C n^{\gamma-1}}{\sqrt{(a+1)(b+1)}} \leq W_{1} \leq \frac{C n^{\gamma-1}}{\sqrt{(1-b)(1-a)}}-\frac{C}{n \sqrt{a+1}}
$$


and

$$
\frac{C}{n \sqrt{a+1}}+\frac{C}{n \sqrt{1-b}}-C n^{\gamma-1} \leq W_{2} \leq \frac{C n^{\gamma-1}}{\sqrt{(a+1)(b+1)}}+\frac{C n^{\gamma-1}}{\sqrt{(1-b)(1-a)}} .
$$

Using that for $\mathbf{y} \in \mathscr{Y}_{L}$ the number of eigenvalues in any interval of size at least $n^{\gamma} N^{-1}$ (before rescaling) is approximated by the semicircle law with a precision $n^{-\gamma / 3}$ (see (4.16)), we get

$$
W_{1}=\varrho_{0} \int_{-Y}^{-1-X} \frac{\mathrm{d} y}{\sqrt{(a-y)(b-y)}}-\varrho_{0} \int_{1+X}^{Y} \frac{\mathrm{d} y}{\sqrt{(a-y)(b-y)}}+O\left(n^{-\gamma / 3} \log n\right)
$$

and

$$
W_{2}=\varrho_{0} \int_{-Y}^{-1-X}\left[\frac{y}{\sqrt{(a-y)(b-y)}}+1\right] \mathrm{d} y-\varrho_{0} \int_{1+X}^{Y}\left[\frac{y}{\sqrt{(a-y)(b-y)}}-1\right] \mathrm{d} y+1+O\left(n^{-\gamma / 3} \log n\right) .
$$

Here we also used that

$$
\sup _{a, b \in[-1,1]} \int_{1+X}^{Y} \frac{\mathrm{d} y}{\sqrt{(a-y)(b-y)}} \leq C \log n
$$

and

$$
\sup _{a, b \in[-1,1]} \int_{1+X}^{Y}\left[\frac{y}{\sqrt{(a-y)(b-y)}}-1\right] \leq C \log n .
$$

Let $u=\frac{1}{2}(a+b)$ and $v=\frac{1}{2}(b-a)$ and we can assume, by symmetry, that $u \geq 0$. Then we can change variables in the integrals in (F.17)

$$
\begin{aligned}
W_{1} & =\varrho_{0} \int_{-Y-u}^{-1-X-u} \frac{\mathrm{d} y}{\sqrt{y^{2}-v^{2}}}-\varrho_{0} \int_{1+X-u}^{Y-u} \frac{\mathrm{d} y}{\sqrt{y^{2}-v^{2}}}+O\left(n^{-\gamma / 3} \log n\right) \\
& =\varrho_{0} \int_{Y-u}^{Y+u} \frac{\mathrm{d} y}{\sqrt{y^{2}-v^{2}}}-\varrho_{0} \int_{1+X-u}^{1+X+u} \frac{\mathrm{d} y}{\sqrt{y^{2}-v^{2}}}+O\left(n^{-\gamma / 3} \log n\right) .
\end{aligned}
$$

The first term is of order $Y^{-1}$ and thus negligible. Thus, from the lower bound in (F.15), we have

$$
\varrho_{0} \int_{1+X-u}^{1+X+u} \frac{\mathrm{d} y}{\sqrt{y^{2}-v^{2}}} \leq \frac{C n^{\gamma-1}}{\sqrt{(a+1)(b+1)}}-\frac{C}{n \sqrt{1-b}}+C n^{-\gamma / 3} \log n .
$$

Estimating $y^{2}-v^{2} \leq(1+X+a)(1+X+b)$ on the integration domain, we get

$$
\begin{gathered}
\frac{C u \varrho_{0}}{\sqrt{(1+X+a)(1+X+b)}} \leq \frac{C n^{\gamma-1}}{\sqrt{(a+1)(b+1)}}+C n^{-\gamma / 3} \log n, \\
C u \varrho_{0} \leq C n^{\gamma-1}\left(1+\frac{X}{a+1}\right)+C n^{-\gamma / 3} \log n .
\end{gathered}
$$

Clearly $a+1 \geq 2 u$, thus

$$
C u \varrho_{0} \leq C n^{\gamma-1}\left(1+\frac{X}{u}\right)+C n^{-\gamma / 3} \log n,
$$


from which it follows that

$$
u \leq C n^{-\gamma / 3} \log n
$$

if $\gamma \leq 1 / 2$. The case $u \leq 0$ is treated similarly, thus we have shown that

$$
|a+b| \leq C n^{-\gamma / 3} \log n
$$

Now we consider the $W_{2}$ and assume again that $u \geq 0$. With the same change of variables as above, we have

$$
\begin{aligned}
W_{2} & =\varrho_{0} \int_{-Y-u}^{-1-X-u}\left[\frac{y+u}{\sqrt{y^{2}-v^{2}}}+1\right] \mathrm{d} y-\varrho_{0} \int_{1+X-u}^{Y-u}\left[\frac{y+u}{\sqrt{y^{2}-v^{2}}}-1\right] \mathrm{d} y+1+O\left(n^{-\gamma / 3} \log n\right) \\
& =\varrho_{0} \int_{-Y-u}^{-1-X-u}\left[\frac{y}{\sqrt{y^{2}-v^{2}}}+1\right] \mathrm{d} y-\varrho_{0} \int_{1+X-u}^{Y-u}\left[\frac{y}{\sqrt{y^{2}-v^{2}}}-1\right] \mathrm{d} y+1+O\left(n^{-\gamma / 3}(\log n)^{2}\right),
\end{aligned}
$$

where we used (F.19) and

$$
\int_{-Y-u}^{-1-X-u} \frac{1}{\sqrt{y^{2}-v^{2}}} \mathrm{~d} y \leq C \log n \quad \text { and } \quad \int_{1+X-u}^{Y-u} \frac{1}{\sqrt{y^{2}-v^{2}}} \mathrm{~d} y \leq C \log n .
$$

The integrals on the r.h.s of (F.20) can be explicitly computed:

$$
\begin{gathered}
\int_{1+X-u}^{Y-u}\left[\frac{y}{\sqrt{y^{2}-v^{2}}}-1\right] \mathrm{d} y=\left.\frac{-v^{2}}{\sqrt{y^{2}-v^{2}}+y}\right|_{1+X-u} ^{Y-u}=\frac{v^{2}}{\sqrt{(1+X-u)^{2}-v^{2}}+1+X-u}+O\left(Y^{-1}\right), \\
\quad \int_{-Y-u}^{-1-X-u}\left[\frac{y}{\sqrt{y^{2}-v^{2}}}+1\right] \mathrm{d} y=\frac{-v^{2}}{\sqrt{(1+X+u)^{2}-v^{2}}+1+X+u}+O\left(Y^{-1}\right),
\end{gathered}
$$

thus we have

$$
W_{2} \geq 1-2 \varrho_{0} v^{2}-C n^{-\gamma / 3}(\log n)^{2}-C Y^{-1} \geq 1-\frac{2}{\pi}-C n^{-\gamma / 3}(\log n)^{2}
$$

by using that $v^{2} \leq 1$ and $\varrho_{0} \leq \pi^{-1}$ (see (2.7)). Combining this estimate with the upper bound in (F.16), we have

$$
1-\frac{2}{\pi}-C n^{-\gamma / 3}(\log n)^{2} \leq \frac{C n^{\gamma-1}}{\sqrt{(a+1)(b+1)}}+\frac{C n^{\gamma-1}}{\sqrt{(1-b)(1-a)}} \leq \frac{C n^{\gamma-1}}{a+1}+\frac{C n^{\gamma-1}}{1-b}
$$

by using $a<b$. Therefore either $a+1$ or $1-b$ is smaller than $C n^{\gamma-1}$, but then by using (F.19) we obtain that both of them are smaller then $C n^{-\gamma / 3} \log n$. This completes the proof of Lemma F.1. 


\section{References}

[1] Anderson, G., Guionnet, A., Zeitouni, O.: An Introduction to Random Matrices. Studies in advanced mathematics, 118, Cambridge University Press, 2009.

[2] Bakry, D., Émery, M.: Diffusions hypercontractives. in: Séminaire de probabilités, XIX, 1983/84, 1123 Lecture Notes in Mathematics, Springer, Berlin, 1985, 177-206. MR0889476

[3] Ben Arous, G., Péché, S.: Universality of local eigenvalue statistics for some sample covariance matrices. Comm. Pure Appl. Math. LVIII. (2005), 1-42. MR2162782

[4] Bobkov, S. G., Götze, F.: Exponential integrability and transportation cost related to logarithmic Sobolev inequalities. J. Funct. Anal. 163 (1999), no. 1, 1-28. MR1682772

[5] Borwein, P., Erdélyi, T.: Polynomials and Polynomial Inequalities. Springer, 1995 MR1367960

[6] Boutet de Monvel, A., Khorunzhy, A.: Asymptotic distribution of smoothed eigenvalue density. II. Wigner random matrices. Random Oper. and Stoch. Equ., 7 No. 2, (1999), 149-168. MR1689027

[7] Brézin, E., Hikami, S.: Correlations of nearby levels induced by a random potential. Nucl. Phys. B 479 (1996), 697-706, and Spectral form factor in a random matrix theory. Phys. Rev. E 55 (1997), 4067-4083. MR1418841

[8] Davies, E.B.: The functional calculus. J. London Math. Soc. (2) 52 (1) (1995), 166-176. MR1345723

[9] Deift, P: Orthogonal polynomials and random matrices: a Riemann-Hilbert approach. Courant Lecture Notes in Mathematics 3, American Mathematical Society, Providence, RI, 1999 MR1677884

[10] Dyson, F.J.: Statistical theory of energy levels of complex systems, I, II, and III. J. Math. Phys. 3, 140-156, 157-165, 166-175 (1962). MR0143556

[11] Dyson, F.J.: A Brownian-motion model for the eigenvalues of a random matrix. J. Math. Phys. 3, 1191-1198 (1962). MR0148397

[12] Esposito, R., Marra, R., Yau, H.-T.: Navier-Stokes equation for stochastic particle systems on the lattice. Commun. Math. Phys. (1996) 182, no.2, 395-456. MR1447299

[13] Erdős, L., Schlein, B., Yau, H.-T.: Semicircle law on short scales and delocalization of eigenvectors for Wigner random matrices. Ann. Probab. 37, No. 3, 815-852 (2008) MR2537522

[14] Erdős, L., Schlein, B., Yau, H.-T.: Local semicircle law and complete delocalization for Wigner random matrices. Commun. Math. Phys. 287, 641-655 (2009) MR2481753

[15] Erdős, L., Schlein, B., Yau, H.-T.: Wegner estimate and level repulsion for Wigner random matrices. Int. Math. Res. Not., Vol. 2010, No. 3, 436-479 (2010).

[16] Erdős, L., Péché, S., Ramirez, J., Schlein, B., Yau, H.-T.: Bulk Universality for Wigner Matrices. To appear in CPAM. (http://arxiv.org/abs/0905.4176) 
[17] Gradshteyn, I.S., Ryzhik, I.M.: Table of Integrals, Series and Products. Academic Press, 1979. MR1773820

[18] Guionnet, A., Zeitouni, O.: Concentration of the spectral measure for large matrices. Electronic Comm. in Probability 5 (2000) Paper 14. MR1781846

[19] Guionnet, A.: Large random matrices: Lectures on Macroscopic Asymptotics. École d'Eté de Probabilités de Saint-Flour XXXVI-2006. Springer. MR2498298

[20] Guo, M.Z., Papanicolaou, G.C., Varadhan, S.R.S.: Nonlinear diffusion limit for a system with nearest neighbor interactions. Commun. Math. Phys. 118 (1988) no.1, 31-59. MR0954674

[21] Johansson, K.: Universality of the local spacing distribution in certain ensembles of Hermitian Wigner matrices. Comm. Math. Phys. 215 (2001), no.3. 683-705. MR1810949

[22] Ledoux, M.: The concentration of measure phenomenon. Mathematical Surveys and Monographs, 89 American Mathematical Society, Providence, RI, 2001. MR1849347

[23] Levin, E., Lubinsky, S. D.: Universality limits in the bulk for varying measures. Adv. Math. 219 (2008), 743-779. MR2442052

[24] Levin, E., Lubinsky, S. D.: Orthogonal polynomials for exponential weights. Springer 2001. MR1840714

[25] Mehta, M.L.: Random Matrices. Academic Press, New York, 1991. MR1083764

[26] Pastur, L., Shcherbina M.: Bulk universality and related properties of Hermitian matrix models. J. Stat. Phys. 130 (2008), no.2., 205-250. MR2375744

[27] Quastel, J., Yau, H.T.: Lattice gases, large deviations and the incompressible Navier-Stokes equations. Ann. of Math. (2) 148 (1998), no.1, 51-108. MR1652971

[28] Soshnikov, A.: Universality at the edge of the spectrum in Wigner random matrices. Comm. Math. Phys. 207 (1999), no.3. 697-733. MR1727234

[29] Tao, T., Vu, V.: Random matrices: universality of local eigenvalue statistics. Preprint arxiv:0906.0510.

[30] Wigner, E.: Characteristic vectors of bordered matrices with infinite dimensions. Ann. of Math. 62 (1955), 548-564. MR0077805 